\title{
AUTOMATIC EXTRACTION OF DIGITAL ELEVATION \\ MODELS FROM IKONOS IN-TRACK STEREO IMAGES
}

by

\section{Xu Sun}

B.Eng., Wuhan University, China, 1994

A thesis presented to Ryerson University

in partial fulfillment of the

requirements for the degree of

Master of Applied Science

in the Program of Civil Engineering

Toronto, Ontario, Canada, 2005

(C) Xu Sun, 2005 


\section{UMI Number: EC53768}

\section{INFORMATION TO USERS}

The quality of this reproduction is dependent upon the quality of the copy submitted. Broken or indistinct print, colored or poor quality illustrations and photographs, print bleed-through, substandard margins, and improper alignment can adversely affect reproduction.

In the unlikely event that the author did not send a complete manuscript and there are missing pages, these will be noted. Also, if unauthorized copyright material had to be removed, a note will indicate the deletion.

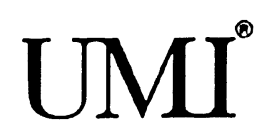

\section{UMI Microform EC53768}

Copyright 2009 by ProQuest LLC

All rights reserved. This microform edition is protected against unauthorized copying under Title 17, United States Code.

ProQuest LLC

789 East Eisenhower Parkway

P.O. Box 1346

Ann Arbor, MI 48106-1346 


\section{Declaration}

I hereby declare that I am the sole author of this thesis.

I authorize Ryerson University to lend this thesis to other institutions or individuals for the purpose of scholarly research.

$\mathrm{Xu}$ Sun

Department of Civil Engineering

Ryerson University

I further authorize Ryerson University to reproduce this thesis by photocopying or by other means, in total or in part, at the request of other institutions or individuals for the purpose of scholarly research.

Xu Sun

Department of Civil Engineering

Ryerson University 


\section{Borrower's Page}

Ryerson University requires the signatures of all persons using or photocopying this thesis.

Please sign below, and give address and date.

\begin{tabular}{|c|c|c|c|}
\hline Name of Borrowers & Date & Address & Signature \\
\hline & & & \\
\hline & & & \\
\hline & & & \\
\hline & & & \\
\hline & & & \\
\hline & & & \\
\hline & & & \\
\hline & & & \\
\hline & & & \\
\hline & & & . \\
\hline & & & \\
\hline & & & \\
\hline
\end{tabular}




\title{
Automatic Extraction of Digital Elevation Models From IKONOS In-track
}

\author{
Stereo Images \\ Xu Sun \\ Master of Applied Science, 2005 \\ Department of Civil Engineering \\ Ryerson University
}

\begin{abstract}
This thesis addresses the topic of the extraction of Digital Elevation Models (DEMs) from the in-track stereo images acquired by IKONOS satellite. Research on this topic is mainly motivated by the need of DEMs in transportation and the potential use of very high resolution satellite stereo images to replace the traditional aerial photography to generate the DEMs that may be used for preliminary planning and location issues, limiting expensive and time consuming photogrammetry work to the final alignment corridor.
\end{abstract}

In this thesis, two methods for DEM extraction from IKONOS stereo images using a modified Rational Function Model (RFM) and the 3D physical model developed at the Canada Centre for Remote Sensing (CCRS) are used and the accuracy of the DEMs generated using these two models are evaluated. The nominal accuracy of ground points determined with the vendor-supplied RPCs is evaluated and systematic biases are found. A significant improvement in the DEM accuracy is made by removing the biases in both the image and ground domain with the information of ground control. DEMs are automatically generated by a chain of processes using the PCI Geomatica OrthoEngine software package with the refined RFM and the 3D physical model, respectively. The DEMs from these two methods are then compared in a desktop ERDAS Imagine environment and the accuracy of the DEMs is evaluated by comparing the extracted DEMs with the DEM from airphotos. The DEMs generated using different mathematical models have a very good consistence and more than $97 \%$ of the difference between the generated DEMs and the DEM from airphotos is between $-2 \mathrm{~m}$ to $2 \mathrm{~m}$. 


\section{Acknowledgements}

First of all, I would like to express my great appreciation and thanks to my supervisor, Professor Dr. Jonathan $\mathrm{Li}$, for his valuable advices and ideas as well as constructive suggestions and productive comments on my thesis. I also thank him for providing me the great opportunity to continue my study in the field of photogrammetry and remote sensing in Canada and to work with him as a research assistant during my years at Ryerson University. I would also like to thank Professor Dr. Michael A. Chapman, my co-supervisor, for his encouragement, patience and critical guidance. His innovative suggestions and valuable ideas had a great influence on my thesis study. I greatly appreciate their efforts to provide me financial supports throughout my course and thesis studies. Without their support, I would never complete this thesis work.

This study was sponsored by Ontario Ministry of Transportation (MTO) under the "Satellite-derived DEMs: comparison of accuracy, cost and time with airphotos" project. I would like to thank Ronald E. Berg, Deputy Chief Surveyor, and his colleagues Rod Scott and Hassan Anys in the MTO's Geomatics Office for their valuable discussion and suggestion. I would also express my thanks to Anthony P. Sani, President of the Sani-International Technology Advisors Inc., Toronto, Ontario, for giving a chance to work in his company for the GCPs collection and DEM evaluation. I also attribute my accomplishment to Professors Dr. Songnian Li, Dr. Mohamed Lachemi and other faculty and staff members in the Department of Civil Engineering, for their help and support. I wish to specially thank 
Professor Dr. Kunquan Lan, Department of Mathematics, for his friendly help during my study. Many thanks go to Desmond Rogan and Valle Domenic for their technical assistance for solving computer and software problems. Many thanks are also extended to Leah Stanwyk, Kim Kritzer and Dianne Mendonca for their administrative support.

The School of Graduate Studies of Ryerson University is acknowledged for providing me the Ryerson Graduate Scholarships and the opportunity to conduct my research at Ryerson University from 2003 to 2005. Ontario Ministry of Transportation and Natural Sciences and Engineering Research Council of Canada (NSERC) are also acknowledged for providing partially financial support.

I also wish to thank my fellow graduate students in the Program of Civil Engineering at Ryerson University, Yu Li, Haibin Liu, Zheng Chang, Hui Jin, Ruiqiu Li and Hongmei Zhao for their help. The study time with them always recalls my beautiful and happy recollections.

Last, but not least, I wish to express my gratitude, from my deepest heart, to my parents for their unconditional love and support, and to my husband, Wensong $\mathrm{Hu}$, for his endless love, patience, and encouragement. Without his help and understanding, I would never complete this thesis. 


\section{Table of Contents}

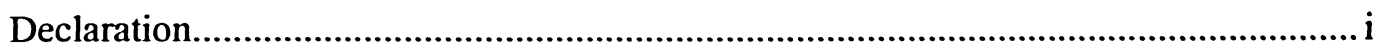

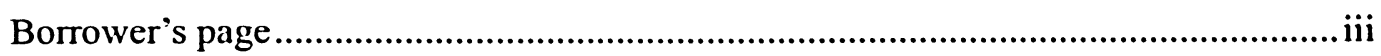

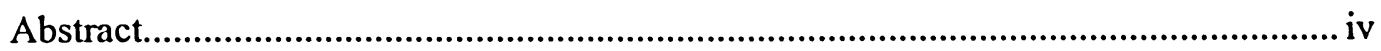

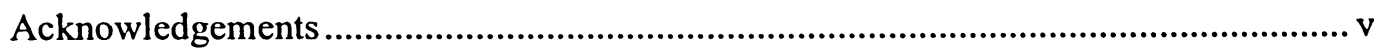

Table of Contents ...................................................................................... vii

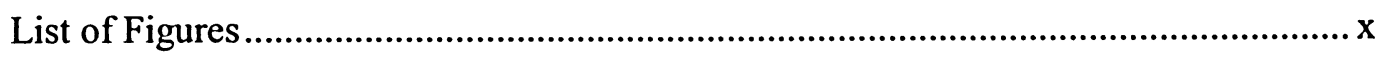

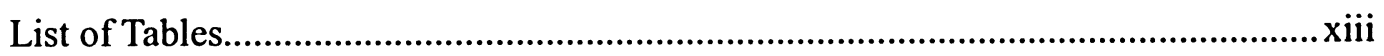

List of Abbreviations.............................................................................. xivv

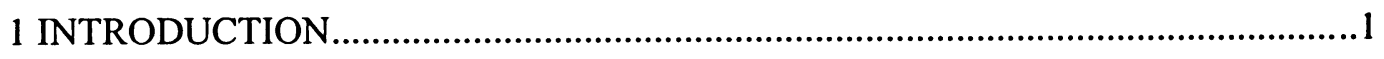

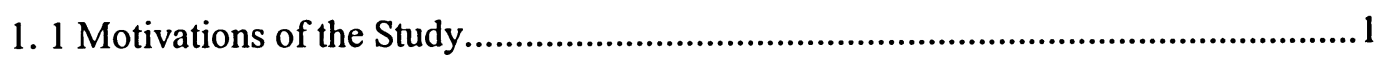

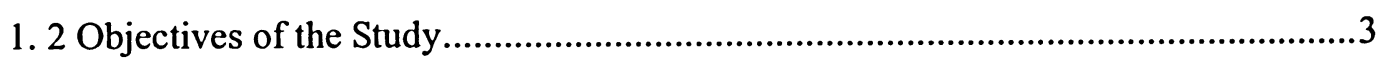

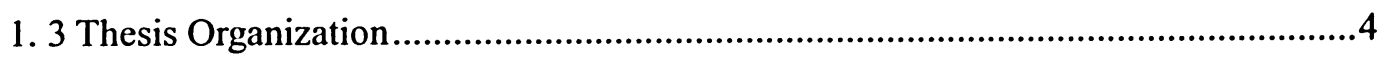

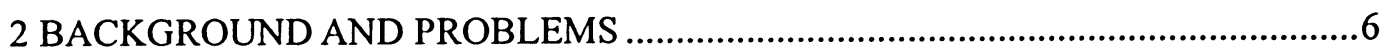

2.1 Definition of DEM...........................................................6

2.2 Methods for DEM Generation...............................................8

2.2.1 Photogrammetric Methods using Aerial Photographs......................8

2.2.2 Methods using IFSAR Data.........................................

2.2.3 Method using Lidar Data........................................... 10

2.2.4 Methods Using Satellite Stereo Images................................11 
2.3 DEM Accuracy Assessment

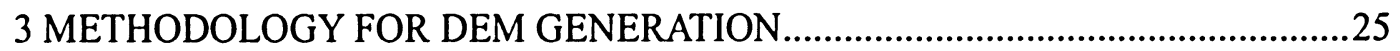

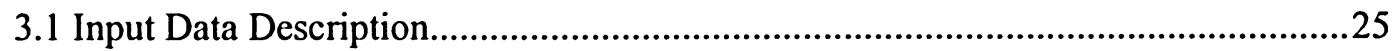

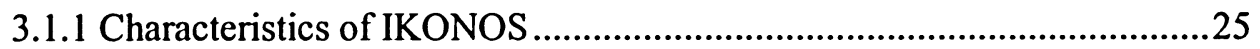

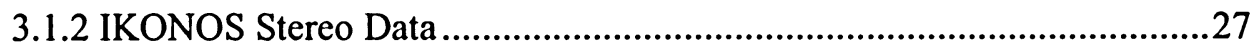

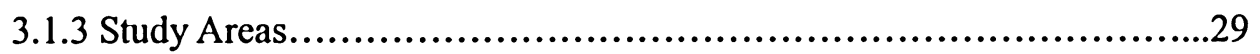

3.2 Strategy for DEM Generation.................................................31

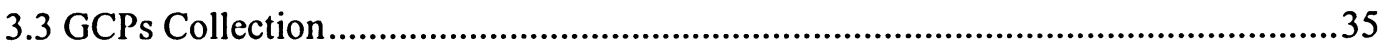

3.4 Sensor Models of IKONOS Imagery …………….................................................

3.4.1 Rational Function Model.......................................................................37

3.4.2 3D Physical Model..............................................................................54

3.4.3 Accuracy Assessment of the Sensor Model..............................................58

3.5 Automatic Image Matching ….............................................................................6

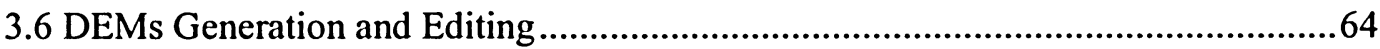

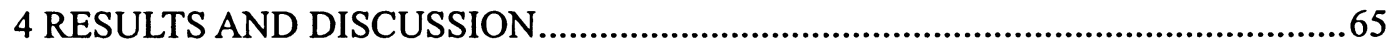

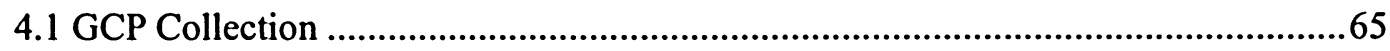

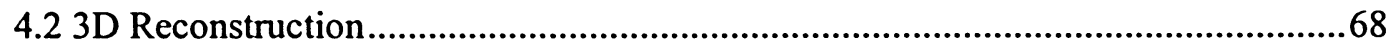

4.2.1 3D Reconstruction Using RFM .............................................................69

4.2.2 3D Reconstruction Using the 3D Physical Model ....................................84

4.3 Epipolar Images and DEM Generation ..................................................................8 


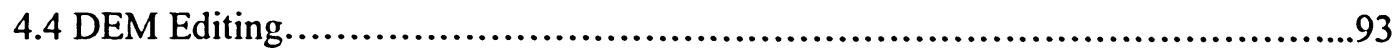

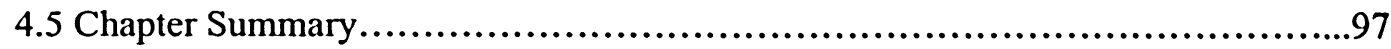

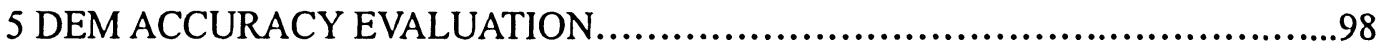

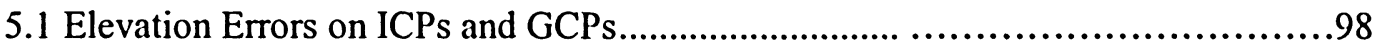

5.2 Comparison of the DEMs Extracted Using Different Sensor Models .................... 100

5.3 Comparison of Extracted DEMs with "True" Terrain ................................................ 106

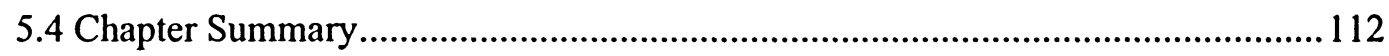

6 CONCLUSIONS AND RECOMMENDATIONS...............................114

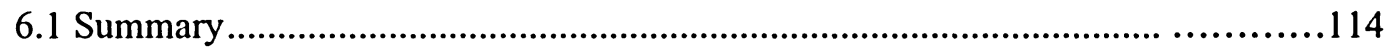

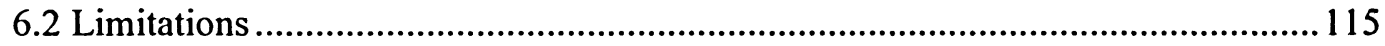

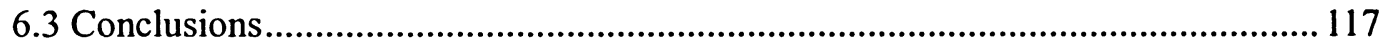

6.4 Recommendations for Future Research.......................................................118

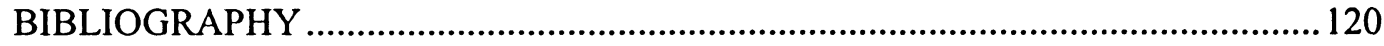




\section{List of Figures}

Figure 3-1 Pushbroom camera (Grodecki and Dial, 2001)...............................26

Figure.3-2 Same date in-track IKONOS stereo data acquisition (Dial and Grodecki, 2003)....29

Figure 3-3 Left image of the IKONOS stereo pair acquired on 20 January 2004, north of Toronto, Ontario, Canada............................................................30

Figure 3-4 Processing steps for the DEM extraction from IKONOS stereo images and its accuracy evaluation. .34

Figure 3-5 Processing steps of 3D reconstruction using the RFM model.....................54

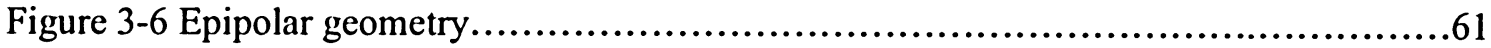

Figure 4-1 An example of the selection of one GCP....................................66

Figure 4-2 GCPs distributed on the central subsection of the left image of the IKONIOS stereo pair.

Figure 4-3 Error vectors of (a) planimetry and (b) elevation at ICPs, calculated using vendor-supplied RPCs.

Figure 4-4 Bias vectors of image coordinates of (a) left and (b) right image, calculated using vendor-supplied RPCs.

Figure 4-5 Error vectors in (a) planimetry and (b) elevation of GCPs (marked in black) and ICPs (marked in gray) using calculated RPCs.

Figure 4-6 Error vectors in (a) planimetry and (b) elevation of ICPs using 1 GCP in the centre to remove the bias in image domain.

Figure 4-7 Error vectors in (a) planimetry and (b) elevation of ICPs using 3 GCPs at the edge to remove the bias in image domain.

Figure 4-8 Error vectors in (a) planimetry and (b) elevation of ICPs using 4 GCPs at the corner to remove the bias in image domain

Figure 4-9 Coefficient differences between the refined and original RPCs of the $\mathrm{x}$ numerator in the left image. 
Figure 4-10 Coefficient differences between the refined and original RPCs of the y numerator in the left image. 80

Figure 4-11 Coefficient differences between the refined and original RPCs of the $\mathrm{x}$ numerator in the right image.

Figure 4-12 Coefficient differences between the refined and original RPCs of the y numerator in the right image. .81

Figure 4-13 Error vectors in (a) planimetry and (b) elevation of ICPs using 1 GCP in the centre to remove the bias in ground domain.

Figure 4-14 Error vectors in (a) planimetry and (b) elevation of ICPs using 3 GCPs at the edge to remove the bias in ground domain.

Figure 4-15 Error vectors in (a) planimetry and (b) elevation of ICPs using 7 GCPs at the corner to remove the bias in ground domain.

Figure 4-16 RMSE values for ICPs from the least-squares bundle adjustment computed with GCPs varying from 6-60 for the study area. 85

Figure 4-17 Distribution of the GCPs and ICPs used in the 3D physical model bundle adjustment.

Figure 4-18 Left epiporlar image of the IKONOS in-track stereo images, north Toronto, Ontario, Canada, 20 January 2004.

Figure 4-19 Right epiporlar image of the IKONOS in-track stereo images, north Toronto, Ontario, Canada, 20 January 2004.

Figure 4-20 Epipolar DEM generated using the refined RFM. 90

Figure 4-21 Epipolar DEM generated using the 3D physical model. 91

Figure 4-22 Geocoded DEM derived using the RFM model. .92

Figure 4-23 Geocoded DEM derived using the 3D physical model. .93

Figure 4-24 DEM derived using the 3D physical model after noise removal processing........94

Figure 4-25 DEM derived using the 3D physical model after interpolation. .95

Figure 4-26 DEM derived using the 3D physical model after smoothing. .96 
Figure 5-1 Processing strategy of the generation of difference DEM using ERDAS Imagine V8.7 Spatial Modeler.

Figure 5-2 Difference DEM between the DEM extracted using the RFM with vendor-supplied RPCs and the DEM extracted using the 3D physical model. 102

Figure 5-3 Difference DEM between the DEM extracted using the refined RFM and the DEM extracted using the $3 \mathrm{D}$ physical model. 103

Figure 5-4 Elevation distribution of the difference DEM between the DEM derived using the $3 \mathrm{D}$ physical model and the DEM derived using the refined RFM .105

Figure 5-5 Elevation distribution of the difference DEM between the DEM derived using the 3D physical model and the DEM derived using the RFM with vendor-supplied RPCs.

Figure 5-6 "Ground truth" DEM manually edited from aerial photographs...

Figure 5-7 DEM generated using the 3D physical model

Figure 5-8 DEM generated using the RFM.......................................... 108

Figure 5-9 The DEM after the interpolation processing of the building area. 109

Figure 5-10 Difference DEM between the DEM extracted using the 3D physical model and the "true" terrain

Figure 5-11 Difference DEM between the DEM extracted using the RFM and the "true" terrain. 110

Figure 5-12 Elevation distribution of the difference DEM between the DEM extracted using the $3 \mathrm{D}$ physical model and the "true" terrain.

Figure 5-13 Elevation distribution of the difference DEM between the DEM extracted using the RFM and the "true" terrain. 


\section{List of Tables}

Table 2-1 Terminology of elevation data................................................7

Table $3-1$ IKONOS product levels................................................28

Table 3-2 Specifications of the IKONOS stereo images..................................31

Table 4-1 GCPs with their 3D ground coordinates and 2D image coordinates................66

Table 4-2 RMSE and STDE on the ICPs without GCP...................................69

Table 4-3 RMSE at the GCPs and ICPs using the new calculated RPCs.....................72

Table 4-4 RMSE values of ICPs using different selection of GCPs to remove the bias in image domain............................................................................77

Table 4-5 Computed image biases and RMSE of the ICPs................................78

Table 4-6 RMSE of checkpoints using different selection of GCPs to remove the biases in ground domain...................................................................... 83

Table 4-7 RMSE, average and maximum errors of GCPs and ICPs calculated using the 3D physical model................................................................... 87

Table 5-1 Comparison of elevation differences using different 3D stereo models...............99

Table 5-2 Statistic results of the two sets of difference DEMs on ICPs...................... 104

Table 5-3 Statistical results of the difference between the generated DEMs and the "true" terrain. 


\section{List of Abbreviations}

2D

3D

CCRS

CE90

DEM

DLT

DSM

GCP

HRSC

ICP

LE 90 (95)

Lidar

IFSAR

MSL

NSSDA

OGC

PAN

RFM

RMSE

RPC

STDE

TIN
Two-Dimensional

Three-Dimensional

Canada Centre for Remote Sensing

Circular Error 90

Digital Elevation Model

Direct Liner Transformation

Digital Surface Model

Ground Control Point

High Resolution Stereo Colour

Independent Check Point

Linear Error 90 (95)

Light Detection And Ranging

Interferometer Synthetic Aperture Radar

Mean Sea Level

National Standard for Spatial Data Accuracy

Open Geospatial Consortium

Panchromatic

Rational Function Model

Root Mean Square Error

Rational Polynomial Coefficient

Standard Deviation

Triangulated Irregular Network 


\section{INTRODUCTION}

\subsection{Motivations of the Study}

Characterization of surface topography is necessary for transportation corridor planning. Accurate terrain information is vital in determining alignment and grade. Used in conjunction with volumetric analysis tools, this same information allows estimation of cut and fill operations. Analyzed in light of local geology and hydrology, it can be used to assess landslide potential. Biogeographic analysis can also indicate potential ecological impacts as a result of corridor construction and utilization. Common practice in corridor planning involves the use of surveying and/or photogrammetry to generate topographic information. Data collections by ground surveying require a considerable investment of time and labor and may not be practical for large projects. Crews also face potential hazard exposure in the field. Conversely, the creation of a photographic database for photogrammetry is hindered by considerations of weather, sun angle, and vegetation condition. Consequently, photo acquisition is typically limited to early spring and late fall in Canada. Additionally, while generally not as labor intensive as surveying, photogrammetric methods involve significant time and labor costs. As an adjunct to these methods, the creation of a digital elevation model (DEM) from remotely sensed data can provide savings of both time and labor while accelerating the early planning stages of a project dealing with corridor route alternatives and their potential benefits, impacts, and drawbacks. 
IKONOS, successfully launched on 24 September 1999, was the world's first civilian remote sensing satellite with new high-resolution imaging sensors $(1 \mathrm{~m}$ panchromatic, Pan and $4 \mathrm{~m}$ multispectral) and an off-nadir viewing capability (up to $60^{\circ}$ in any azimuth). Its $360^{\circ}$ pointing capability enables the generation of across-track stereoscopic images from two different orbits as well as of in-track stereoscopic images from the same orbit with a base-to-height ratio $(\mathrm{B} / \mathrm{H})$ of one. Users could then apply traditional three-dimensional (3D) photogrammetric techniques with the stereo images to extract accurate planimetric and elevation information. In fact, same-date in-track stereo data acquisition has a strong advantages over multidate across-track stereo data acquisition because it reduces radiometric image variations (temporal changes, sun illumination, etc.), and thus increases the correlation success rate in any image matching process (Toutin, 2000; 2004). This in-track solution to acquiring stereo data is generally chosen by Space Imaging (the vendor), not only for scientific, but also for operational reasons.

However, rigorous physical sensor models are not always available for satellite sensor orientation, especially for images from high resolution satellites such as IKONOS. Unlike the rigorous physical sensor model, non-rigorous models such as the rational function mode (RFM) need neither the knowledge of the sensor model nor of orbit ephemeris and platform orientation parameters. Applications of such a model to stereo images acquired by IKONOS satellite indicate that relatively accurate geopositioning can be obtained through provision of ground control points (GCPs). 
Space Imaging does not provide the ancillary data such as the position, velocity vectors and angular rates of the IKONOS satellite platform. Therefore, alternative ways of camera modeling need to be employed in order to extract accurate elevation information from IKONOS stereo images. The work presented in this thesis was motivated by the need for efficient methods of automatic extraction of DEMs from IKONOS in-track stereo images for transportation corridor planning GIS database updating in complex urban areas.

\subsection{Objectives of the Study}

The first purpose objective of this study is to develop proper methods for automatically extracting DEMs from IKONOS in-track stereo images. The investigations mainly focus on the evaluation of two different non-rigorous models, the rational function model (RFM) and the 3D physical model developed at the Canada Centre for Remote Sensing (CCRS), Natural Resources Canada, which have been adapted to IKONOS images. The second objective of this study is to evaluate DEM quality when compared to accurate ground truth (high accuracy DEM derived by photogrammetric processing of large-format aerial photographs), and to track the error propagation from the input data to the final DEM and to evaluate the different parameters affecting the accuracy of DEM.

In order to achieve the above objectives, the following key issues are included in this thesis: 
- The collection of GCP coordinates from both the IKONOS stereo images and orthoimage of the study area.

- The 3D reconstruction of the IKONOS stereo pair by using the RFM and the 3D physical model, respectively.

- The automatic image matching of IKONOS in-track stereo images.

- The automatic DEM generation by using PCI Geomatica OrthoEngine.

- The comparison of the DEMs derived using different mathematical models in a desktop ERDAS Imagine environment.

- The comparison of the DEMs derived from IKONOS in-track stereo images to the high accuracy DEM generated from large-format airphotos, which were used for the collection of GCPs.

\subsection{Thesis Organization}

The thesis consists of six chapters and is organized as follows.

Chapter 1 describes the general goal and problems in DEM generation using high-resolution satellite image data and sets forth the purpose of this study.

Chapter 2 presents an overview of the methods used in DEM generation. Details are given to the RFM and the 3D physical model, automatic matching algorithms and the techniques of DEM accuracy assessment. 
Chapter 3 proposes a methodology developed and used in this thesis, including GCP collection, stereo image orientation, stereo image matching, DEM generation, and accuracy assessment.

Chapter 4 presents a case study in the use of proposed methodology to automatically extract DEMs from IKONOS in-track stereo images.

Chapter 5 evaluates the accuracy of the final DEMs by comparing the DEMs generated using different non-rigorous models and also comparing the extracted DEMs with the "true" terrain.

Chapter 6 presents conclusions related to the findings from the study and suggests future work to extend the study. 


\section{BACKGROUND AND PROBLEMS}

With the availability of very high resolution remote sensing satellites such as IKONOS and QuickBird, several researchers around the world have investigated the extraction of elevation data and/or the production of DEMs using different methods (Leberal, 1998; Madsen and Zebker, 1998; Toutin, 2000; 2004). This chapter attempts reviewing the relevant research systematically in order to obtain an overview of research trends and to identify the key achievements of the various approaches. It is not meant to be an exhaustive review of the literature, but more an indication of the theoretical development of, and problems associated with the generation of DEMs from IKONOS stereo images and their 3D accuracy assessment of the derived DEMs.

\subsection{Definition of DEM}

The term Digital Elevation Model has several different meanings. Generally, DEM is a generic term for digital topographic and/or bathymetric data, in all its various forms. It is called a "model" because computers can use such data to model and automatically analyze the Earth's topography in three-dimensions, minimizing the need for labor-intensive human interpretation. Unless specially referenced as a Digital Surface Model (DSM), the generic DEM normally implies elevations of the terrain (bare Earth z-values) void of vegetation and manmade features. This bare-Earth DEM is generally synonymous with a Digital Terrain Model (DTM). All elevations are referenced to a 
horizontal and vertical datum.

Table 2-1 Terminology of elevation data

\begin{tabular}{|c|l|l|}
\hline Terms & \multicolumn{1}{|c|}{ Descriptions } & \multicolumn{1}{|c|}{ Applications } \\
\hline DEM & $\begin{array}{l}\text { Representation of the terrain at } \\
\text { regular spaced intervals in x and y } \\
\text { direction }\end{array}$ & $\begin{array}{l}\text { Hydrology, transportation and } \\
\text { etc. }\end{array}$ \\
\hline DTM & $\begin{array}{l}\text { Similar to DEM, but may also } \\
\text { incorporate the elevation of } \\
\text { significant topographic features on } \\
\text { the land, plus mass points are } \\
\text { irregularly spaced }\end{array}$ & $\begin{array}{l}\text { Better to characterize the true } \\
\text { shape of the bare earth terrain } \\
\text { and superior to standard } \\
\text { DEMs. }\end{array}$ \\
\hline DSM & $\begin{array}{l}\text { Depicts the elevations of the top } \\
\text { surfaces of buildings, trees, } \\
\text { towers, and other features elevated } \\
\text { above the bare earth }\end{array}$ & $\begin{array}{l}\text { Telecommunication planning, } \\
\text { forest management, air safety, } \\
\text { and 3D simulation and fly } \\
\text { through }\end{array}$ \\
\hline TIN & $\begin{array}{l}\text { A set of adjacent, non-overlapping } \\
\text { triangles computed from irregular } \\
\text { spaced points with x/y coordinates } \\
\text { and z-values }\end{array}$ & $\begin{array}{l}\text { TINs are excellent for } \\
\text { calculation of slope, aspect, } \\
\text { surface area and length; } \\
\text { generation of contours }\end{array}$ \\
\hline
\end{tabular}

Table 2-1 presents the terminology associated with elevation data types and their applications. As normally used, a DEM has bare Earth z-values at regularly spaced intervals in $\mathrm{x}$ (Eastings) and $\mathrm{y}$ (Northings). However, grid spacing, datum, coordinate systems, data formats, and other characteristics may vary widely, but normally following alternative specifications. Since the topographic surface can be considered to be a mathematically continuous surface, an infinite number of points may be selected to represent the topography no matter how small an area is. Practically, only a sample can be utilized, and interpolation is used to construct a digital model of the surface by estimating elevation at any location based on the sample $Z$ values. Thus, a DEM used to 
represent a topographic surface should contain adequate elevation and planimetric measurements compatible in number and distribution with the terrain being modeled, so that the elevation of any location can be interpolated accurately for any given application (Ayeni, 1982). All the major difficulties with computer interpolation are caused by insufficient data and observational error (Waston, 1992). Emphasis is, therefore, placed on data capture and storage techniques.

\subsection{Methods for DEM Generation}

Research on DEM generation can be subdivided with respect to its data and methodologies. Methodologies are greatly dependent on the date types that use because available features from the given data are keys to initiate and guide DEM generation in effective and reliable ways.

\subsubsection{Photogrammetric Methods using Aerial Photographs}

Conventional photogrammetric methods for DEM generation can be classified into three categories: (1) Stereocompilation techniques to collect elevation data from film-based aerial photographs using an analytical plotter, or from digitized or digital images using a softcopy photogrammetric workstation, respectively; (2) Automatic techniques to collect elevation data from digitized photographs or digital images by digital correlation with analytical or digital compilation workstations; and (3) Hybrid approaches. 
Many users employ a mix of the above methods and commonly utilize both film-based "hardcopy" and digital imagery compilation stations "softcopy". Most users would say that the majority of new compilation stations purchases are now softcopy, but analytical systems have a very long life and will be used for some time to come.

\subsubsection{Methods using IFSAR Data}

One of the most efficient means of gathering remotely sensed elevation data is by interferometric synthetic aperture radar (IFSAR). IFSAR is a single pass method in which an aircraft utilizes two antennae separated from each other by a known distance in the cross-track direction. As in a single antenna radar system, energy is emitted toward a target and reflections of that energy are received by the antenna. IFSAR represents the slight difference in return times between the two receivers as a phase shift which can be processed ("unwrapped") to calculate a distance from the platform to the target. By factoring in precise aircraft positional data, elevation information is derived and a digital representation of the target surface produced. Traditional stereoscopic measurement of the "parallax" or relative displacement that an object has from two stereo images, is proportional to the height of the object and the separation between the two imaging points. For IFSAR systems, the parallax is the range difference from a point to the two observing antennas. Useful topography measurements are possible when the observed parallax is measurable for height variations of interest. The key to extending the range of useful stereoscopic observations is that parallax measurements are obtained by measuring the phase difference between signals received by the two 
IFSAR antennas. Distance measurements are related to phase measurements by converting the distance to units of wavelength and recalling each wavelength corresponds to $2 \pi$ radians or $360^{\circ}$ of phase. If the received signals from the two antennas are combined coherently for each imaged point to measure the phase difference, then the system forms an interferometric SAR. Here, the interferometric phase difference is essentially related to the geometric path length difference to the image point, which depends on the topography, the phase difference can then be converted into an altitude for each image point.

\subsubsection{Methods using Lidar Data}

Since the mid 1990s, airborne laser scanning or topographic Lidar (Light Detection And Ranging) has provided the surveying and mapping professions additional capabilities in locating and depicting spatial information cost-effectively and quickly. The active nature of the technology allows operators to identify buildings and drainage patterns that may, for example, affect the location of a planned highway when access to private property or visibility through overhead vegetation make conventional methodologies impractical. Since the raw data provided are inherently referenced horizontally and vertically, topographic Lidar reduces the requirement of cost and time to include numerous ground survey points in processing and the development of digital terrain models. While Lidar will not always replace traditional methods of data capture, it is continuing to broaden the capabilities of traditional surveying and mapping companies. 
A lidar system is a complex package of electronic components. Several difference technologies have been married into a complicated technological unit. Laser technology has been used in lidar system to measure and range distances. With a light sensitive receiver tuned to the same frequency as the emitting diode ( a sort of light bulb which produces a specific frequency of light energy), and a very accurate clock, the length of time a short burst or pulse of light energy takes to travel from the light source to an object and then be reflected back to the receiver may be calculated. The total travel time is divided by two and multiplied by the speed of light, resulting in the distance from the light source to the object, which is highly related to the topograph.

\subsubsection{Methods Using Satellite Stereo Images}

The automatic extraction of DEMs from satellite image data is an important task for various mapping applications. Due to its high spatial resolution, IKONOS imagery has the potential for large-scale topographic mapping. Over the past several years, numerous publications (Dial and Grodecki, 2002; Tao and Hu, 2002; Ager, 2003; Di et al., 2003; Toutin, 2003) have demonstrated the high geometric accuracy of IKONOS satellite imagery. Dial and Grodecki (2002) found that the absolute accuracy of an uncontrolled IKONOS image is, on average, $4 \mathrm{~m}$ Root Mean Square Error (RMSE) per axis, and the introduction of as few as one GCP can improve the accuracy to the 1-2 $\mathrm{m}$ level.

The investigations on the use of IKONOS stereo images for the generation of DEMs 
focus on two aspects. One is the alternative senor orientation and geopositioning models for metric applications of IKONOS imagery. The other is the assessment of the planimetric and vertical accuracy of the DEMs extracted from IKONOS stereo pair with or without GCPs.

Considerable investigations have been reported concerning the geometric processing and the accuracy attainable using IKONOS imagery by various methods of processing. Before the actual images were available, Li (1998) discussed the potential accuracy of IKONOS imagery using basic photogrammetry principles. Zhou and Li (2000) used simulated $1 \mathrm{~m}$ resolution IKONOS stereo images and the pushbroom imaging principle to estimate the potential accuracy of ground point determination and reported and expected accuracy of 2 to $3 \mathrm{~m}$. After the successful launch and deployment of IKONOS-2 satellite on September 24, 1999, Space Imaging has refused to release information on the sensor model of IKONOS, as well as data on the precise in-flight position and attitude of the imaging sensor which means that a large number of photogrammetric parameters are unknown and not readily determinable from the imagery alone. At the same time, the very long focal length and narrow angel of view $\left(0.93^{\circ}\right)$ and swath $(\sim 11 \mathrm{~km})$ will likely make an orbital resection unstable. The alternative sensor orientation and geopositioning models for IKONOS imagery have been investigated by numerous researchers over these years. The research on spaceborne sensor models lies on two main aspects. One is the RFM and the other is the parametric sensor model, 3D physical model, developed at the CCRS. This model was 
later implemented in the PCI Geomatica OrthoEngine software.

\section{Rational Function Model}

The Rational Function Model, which is also called Rational Polynomial Coefficient (RPC) camera models, is derived from the physical IKONOS sensor model to describe the object-image geometry. RPC models transform 3D object-space coordinates into 2D image space coordinates. The use of the RPC model to 'replace' the rigorous physical sensor models has been in practice for over a decade (Paderes et al., 1989). The RFM, was initially used in the US military intelligence community. It has recently drawn considerable interest in the civilian remote sensing and Geographical Information System (GIS) community, mainly due to the trend that some commercial high-resolution satellite imaging data such as IKONOS and QuickBird are supplied with RPCs (Cheng and Toutin, 2000) instead of rigorous sensor models. The reason is that for high-resolution pushbroom sensors, the physical camera model, based on the interior and exterior geometry and other physical properties of the sensor, becomes very complicated. Whereas, the RFM could assist the users of the high-resolution satellite data due to its high fitting capability and simplicity, and also makes the photogrammetric interoperability of imagery from different vendors possible. At the same time, this strategy of providing the RPC model instead of the rigorous one, helps the vendors of the data return the information about the sensor, as it is not possible or practical to derive the physical sensor parameters from the RFM. The publications to researchers, developers and users are mainly available in the last few years (Madani, 
1999; Dowman and Dolloff, 2000; Yang, 2000; Tao and Hu, 2001). The RFM has been successfully used for the terrain extraction, orthorectification, and feature extraction task. Dial and Grodecki (2002) also outlined the RPC block adjustment technique and the general discussion of the RFM can be found in Whiteside (1997) and Tao and Hu (2001).

The least-squares solution to the nonlinear RFM has been derived and described in Tao and $\mathrm{Hu}(2001)$ and $\mathrm{Hu}$ and $\mathrm{TaO}$ (2002). There are number of properties and accuracy assessment on the use of the RFM for replacing the rigorous sensor model reported in Tao and Hu (2001), Yang (2000) and Dowman and Dolloff (2000). Tao and Hu (2001) reported that, when using the terrain-independent scenario, the RFM yields a worse case error below 0.06 pixel for SPOT imagery compared with its rigorous model. For IKONOS imagery, the RFM differs from its rigorous model at a worse case error of 0.04 pixel. Numerous tests have shown that the RFM can approximate the rigorous sensor models with no distinguishable loss of accuracy and also retains the full capability of performing photogrammetric processing in the absence of the physical sensor model.

The use of RFM for image rectification was discussed in Yang (2000) and Tao and Hu (2001). The RFM based 3D reconstruction has been implemented in some softcopy photogrammetric software packages (Paderes et al., 1989; Madani, 1999; ERDAS, 2001; Tao and Hu, 2001) for the generation of DEMs using image matching techniques. Yang 
(2000) described an RFM-based iterative procedure to compute the object point coordinates from a stereo pair. In his method, an inverse form of RFM is used to establish the 3D reconstruction. Tao and $\mathrm{Hu}$ (2002) discussed the 3D reconstruction algorithms based on the inverse and forward RFM forms and compared the performance of these two reconstruction methods using several stereo pairs of aerial photography and IKONOS imagery. In Di et al. (2001) and Baltsavias et al. (2001), procedures similar to the above forward or inverse RFM 3D reconstruction were described with tests using aerial imagery, simulated IKONOS data, high resolution stereo colour (HRSC) imagery, or IKONOS stereo pairs.

When the users have the information of GCPs, they could also improve the existing RFM provided by the vendors. Some researchers also provide the result regarding to this situation. Hu and Tao (2002) proposed an incremental technique to correct the RPCs directly. When the covariance matrix of the RPCs is available, users can achieve a reliable updating by controlling the system sensitivity to new control information in the Kalman filtering process. Fraser et al. (2001) proposed a solution by computing their own parameters for the (relief-corrected) affine projection and (extended) DLT (Direct Linear Transformation) models, and found that these first-order models could yield sub-pixel positioning accuracy because of the very small field of view of IKONOS sensor. Grodecki and Dial (2003) proposed the block adjustment of the RFM, which has proved to be an accurate and effective method by using simulated and real numerical experiments. Their work is important because it shows that by adding some adjustable 
terms that are physically significant, the RFM is proved to be competent for traditional photogrammetric processing. The previous difficulty that RFM was not suitable for direct adjustment by analytical triangulation (OGC, 1999a; Tao and $\mathrm{Hu}, 2001$ ) is conquered perfectly in an alternative way. Fraser and Hanley (2003) introduced two bias parameters to compensate for the horizontal displacement in image space due to the lateral shift of the IKONOS satellite platform and to generate bias-corrected RPCs. One benefit of this direct updating of the coefficients is that the exploitation algorithms developed based on the RFM can be used without the need for any change.

\section{D Physical Model}

The 3D physical model developed at CCRS was originally developed to accommodate the geometry of pushbroom scanners, such as SPOT high resolution visible sensor, and was subsequently adapted as an integrated and unified geometric modeling to geometrically process multisensor images (Toutin, 1995). This 3D physical model applied to different image types is robust and not sensitive to the distribution of GCPs when there is no extrapolation in planimetry and elevation.

More recently, the model has been adapted to IKONOS images by taking into account of the image characteristics and the available information in the metadata file (Toutin and Cheng, 2001; Toutin, 2004)). More details on the 3D physical model and development (collinearity equations), its applicability and results with a large data set of IKONOS images and cartographic data acquired around the world can be found in 
Toutin $(2003 ; 2004)$. A comparison was made between the 3D physical model and the RPC model using IKONOS Geo image (non stereo) of the City of Richmond Hill, Ontario, Canada. The RFM was obtained by fitting to GCPs using the PCI Geomatica OrthoEngine software. It was shown that, when 7 GCPs and 23 independent check points (ICPs) were employed with first-and second-order polynomials, the 3D physical model gave an accuracy of $1.3 \mathrm{~m}$ (RMS) for both $\mathrm{X}$ and $\mathrm{Y}$ at the ICPs. Toutin (2001) presented the results of extensive tests using the 3D physical model to examine 13 panchromatic and multispectral IKONOS Geo images over seven study sites with various terrain conditions. It was concluded that, when GCPs are accurate to less than 1 $\mathrm{m}, 10 \mathrm{GCPs}$ are sufficient to improve the accuracy to 2-3 $\mathrm{m}$. Davis and Wang (2001) presented a detailed assessment of the planimetric accuracy of $1 \mathrm{~m}$ resolution IKONOS orthoimage products using results from three Missouri test sites. The IKONOS Geo images (non stereo) were orthorectified using available NAPP DEMs (10 m and /or 30 m spacing, $2.3 \mathrm{~m}$ vertical accuracy). The planimetric accuracy of the orthorectified images is 2 to $3 \mathrm{~m}$, which is comparable to the high accuracy IKONOS Precision product. The 3D physical model that is available in the PCI Geomatica operational environment can be used to adequately and accurately process stereo images and to extract 3D information. Consequently, with accurate ground data users may produce their own DEMs with its own characteristics (projection, datum, grid spacing), and obtain an accuracy of 3 to $5 \mathrm{~m}$ depending on the land cover. These accuracies can be consistently achieved if the automatic DEM is manually edited. Therefore, this methodology should promote the acquisition and the use of IKONOS in-tract stereo 
data in many applications. Since many cartographic features and fine topographic details are presented in IKONOS images, the DEM is in fact a digital surface model (DSM). It can thus be used as a complementary tool to ortho-images for automatically classifying planimetric features (roads, corridors and power lines), urban areas (streets and houses), and for extracting house or canopy elevation. DEMs extracted from IKONOS in-track stereo data thus become a reality if the data becomes available to the end-users of photogrammetry, mapping and remote sensing communities.

\section{Other Sensor Models}

Besides the two orientation models mentioned above, there are also some other format alternative models for the geometric processing of IKONOS imagery. Other polynomial models, either simpler or more complicated than the RPCs, can be estimated with similar methods using a strict sensor model. Options include non-rational polynomials (e.g., Kratky, 1989; Baltsavias and Stallmann, 1992), interpolation techniques using known object-to-image transformations for regular object or image grids (OGC, 1999) or the Universal Image Geometry Model (OGC, 1999), which is an extension of the RFM model. However, Space Imaging is not currently offering such modeling options. Moreover, users cannot readily employ these alternative models since the IKONOS sensor model has not been released. A discussion of the use of rational functions for photogrammetric restitution was given in Dowman and Dolloff (2000). RPCs can only be estimated by using GCPs and not through a representation of the rigorous sensor model, and this option is provided by some commercial photogrammetric systems. 
Similarly other non-rational polynomial models (e.g., Kratky, 1989; Papapanagiotu and Hatzopoulos, 2000) can be estimated. However, estimation of such polynomial coefficients using GCPs needs many control points that cover the entire planimetric and height range of the scene and this can be very difficult to achieve in practice, as well as being very costly. It can also lead to severe extrapolation errors and possible undulations between GCPs, especially with higher degree polynomials. As will be illustrated, the use of so-called terrain-dependent RPCs (Hu and Tao, 2001) is not likely to be warranted for IKONOS imagery since alternative, straightforward models that yield high accuracy are available.

Besides rational functions, there are further models displaying a favorable level of sensor independence, which can be employed in order to overcome the absolute accuracy limitations of RPCs. Potential approaches that take account to varying degrees of the mixed projective/parallel projection of linear $\mathrm{CCD}$ sensors include the direct linear transformation or DLT (El-Madanili and Novak, 1996; Savopol and Armenakis, 1998), an extended DLT form (Wang, 1999; Fraser et al., 2001, 2002), or the use of piece-wise DLT functions (Yang, 2001). A second option is affine projection (Okamoto et al., 1999; Fraser, 2000). The former is fundamentally a projective model, whereas the latter is better suited to IKONOS' nominally parallel along-scan projection. The very small field of view of the sensor of only $0.93^{\circ}$ leads to the overall projection approaching skew parallel in CCD-line direction (in the scan direction it is parallel), especially over smaller subscene areas. As it happens, both these models may be thought of as linear rational functions. In the case of the affine model, a 
perspective-to-affine transformation of the image may first be warranted in stereo scenes containing moderate to high relief variation, though this is more the case with sensors having a larger field of view than IKONOS, e.g. SPOT.

\section{Stereo Image Matching}

DEM generation requires many processing steps such as camera modeling, stereo image matching, editing and interpolation. All these steps contribute to the quality of DEMs, among which stereo matching is crucial to the accuracy and completeness of a DEM. Stereo matching is a process of finding conjugate points in a stereo image pair. There have been many publications regarding stereo matching techniques for various applications (Dhond and Affarwal, 1989). For stereo matching satellite images taken by linear pushbroom cameras, the use of pyramidal matching (Krupnik, 2000), approximation of linear pushbroom images as perspective images (Tateishi, 1992), the use of least squares correlation matching (Otto and Chau, 1989) have been reported.

In the past decade, the development of image matching theory and algorithms has been particularly successful. However, this field still requires further study in order to increase the reliability of image matching. There have been many algorithms and strategies developed for the sake of promoting the reliability of image matching. Multi-level data structures, or pyramids, have been widely applied in image matching algorithms. This is valuable for both the precision and reliability of correlation. At the 
same time, the correlation speed can also be increased. Most algorithms belong to single point matching, except for multi-point least squares correlation by multi-level grid algorithm ( $\mathrm{Li}, 1989 ; 1991)$. Cycle grid-dynamic window matching technique, proposed by Lue and Nova (1991), is an example of the multi-level grid algorithm. The main weakness of the single point matching method is that it is not capable of taking into consideration the matching results of other neighboring points. This means that the method does not consider the consistence of local matching results.

Another method is developed by Nevatia (1984) to approximate straight lines from the curves that are extracted from images. This method is implemented for stereo matching by Medioni (1985). Mcintosh and Mutch (1988) proposed an effective method that determines the line correspondence directly from the values of a match function. The match function is set up by combining several properties of similarity measure between the line segments in a pair of stereo images. Match function weights, and the threshold value must be chosen very carefully. They use an orientation similarity measure of line segments to decide whether the two lines, one in the left image and other in the right image, are a potential match pair.

\subsection{DEM Accuracy Assessment}

The accuracy assessment of DEM mainly includes two parts, the horizontal accuracy and the vertical accuracy. The explanations of both planimetric and elevation accuracy are provided as following: 
As the Z-values of a DEM are referenced to a vertical datum, the accuracy of a single measured elevation point is the closeness of its estimated elevation to a standard or accepted correct value. Sometimes, correct elevations are known, but often the true elevations are unknown. The vertical accuracy of a set of elevation points in a DEM is first determined by its RMSE, the square root of the average of the set of squared differences between dataset coordinate values and coordinates values from an independent source of higher accuracy for identical points. The vertical RMSE is then converted into vertical accuracy at an established confidence level, According to National Standard for Spatial Data Accuracy (NSSDA) (Maune, 2001), if the data have no systematic errors, the $95 \%$ confidence level (LE95) $\left(\right.$ Accuracy $\left._{z}\right)$ is $1.9600 *$ RMSE; for $90 \%$ confidence level (LE90) the conversion would be $1.6449 *$ RMSE (Maune, 2001).

The planimetric accuracy is subject to the same statistics as stated in the vertical accuracy section with the exception that the errors are represented in $2 D(x, y)$ space. The definition of the horizontal accuracy at the $95 \%$ confidence level (Accuracy), according to the NSSDA is $1.7308^{*}$ RMSE. Using circular error probability theory, Accuracy $y_{\mathrm{r}}$ is the radius of a circle of uncertainty such that the true or theoretical location of the point falls within that circle $95 \%$ of the time. 


\subsection{Chapter Summary}

DEMs are in used in many transportation applications. Most of these DEMs are derived from aerial photographs or ground surveys. The manual measurement of DEMs in photogrammetry instruments is expensive and time consuming. Therefore, the method for automatic DEM generation from airborne and spaceborne digital images has been the focus in the study of this field.

Since 1999, the availability of very high resolution satellite images with stereoscopic viewing options has opened new opportunities for satellite based DEM extraction. Indeed, commercial remote sensing satellites have achieved the resolution of $1 \mathrm{~m}$ and better, and also with the character of shorter revision period and large coverage, which let them attract more attention of researchers in photogrammetry and remote sensing. While IKONOS satellite has demonstrated its potential for high accuracy object point determination since it was successfully launched in 1999.

The RFM and 3D physical model are two main models used for the geometric processing of IKONOS imagery. The RFM could be used to do the 3D reconstruction by the vendor-supplied RPCs with no GCP, but the accuracy is relatively low. The 3D physical model has been reported by some researchers with high accuracy when using a small number of GCPs. Some other sensor models are also reported by some researchers to have good geometric accuracy when they are used to process IKONOS imagery. 
From the summary of the background and problem, this study focuses on the use of IKONOS in-track stereo images to automatically extract DEMs, the RFM and 3D physical model will be used as the sensor models for the $3 \mathrm{D}$ reconstruction of IKONOS in-track stereo images. The accuracy of the 3D reconstruction using two different models will be evaluated, and also the accuracy of the final DEMs extracted using different sensor models will be reported. Chapters 3 and 4 will give the details on the methodology used and its implementation. 


\section{METHODOLOGY FOR DEM GENERATION}

This chapter describes the methodology used in the processing steps of the DEM generation of IKONOS stereo pair. Section 3.1 describes the specific features of the IKONOS image data used in this study. Section 3.2 describes the processing steps for DEM extraction from IKONOS in-track stereo images. GCP collection, 3D stereo model computation, automatic image matching, and DEM editing are detailed in Sections 3.3-3.6.

\subsection{Input Data Description}

\subsubsection{Characteristics of IKONOS}

Photogrammetric processing of satellite-borne image is more complicated than aerial photographs. Aerial photographs are acquired at an instant of time with a unique exposure station and orientation. Many satellite cameras (including IKONOS) use linear sensor arrays that scan an image strip while the satellite is moving in its orbit. Consequently the satellite image is acquired over a period of time so that the exposure station and orientation are also functions of time (Dial and Grodecki, 2002). The imaging geometry of IKONOS (see in Figure 3-1) is similar to that of other pushbroom scanners such as MOMS-02/D2 and MOMS-2P or the airborne High Resolution Stereo Camera (HRSC). The IKONOS system is based on a pushbroom scanner with a lens of 
$10 \mathrm{~m}$ focal length that has been folded into a $2 \mathrm{~m}$ length through the use of two additional flat mirrors incorporated into its telescope. The IKONOS satellite simultaneously collects panchromatic and multispectral images, providing the commercial and scientific communities with a dramatic improvement in spatial resolution over previously available remote sensing satellites (e.g., Landsat, SPOT, IRS, MOMS). The IKONOS spacecraft is in a sun synchronous, orbit that is inclined $98.1^{\circ}$ to the equator, $681 \mathrm{~km}$ above the Earth's surface and with an equatorial crossing time of 10:30 a.m. The platform is a pointable, in-track and across-track system enabling ground imaging repeat time of 1.5 days for multispectral data and 2.9 days for panchromatic data. The ground resolution at nadir is $1 \mathrm{~m}$ for panchromatic and $4 \mathrm{~m}$ for multispectral images. The system is equipped with GPS receivers and digital star trackers to establish precise camera position and attitude. Details of the IKONOS satellite and its camera system are given in Gerlach (2000) and Grodecki and Dial (2001).

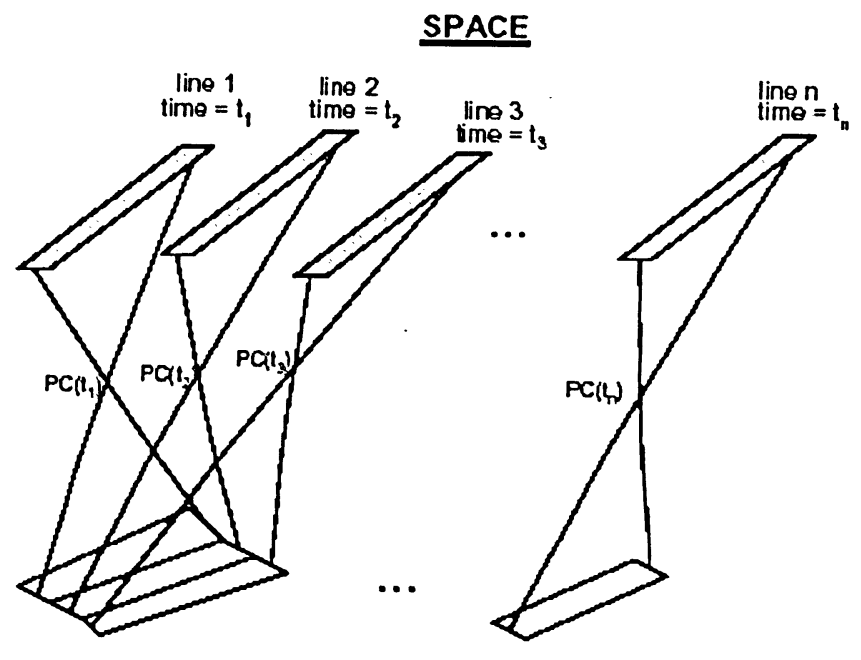

\section{GROUND}

Figure 3-1 Pushbroom camera (Grodecki and Dial, 2001). 


\subsubsection{IKONOS Stereo Data}

There are a number of IKONOS image products with different processing levels including the Geo, Reference, Pro, Precision, and Precision Plus products that have corresponding absolute positioning accuracy (RMSE) of $25,11.8,4.8,1.9$, and $0.9 \mathrm{~m}$, respectively (Space Imaging, 2005. IKONOS Imagery Products and Product Guide, http://www.spaceimaging.com/products/imagery.htm, last accessed on 8 April 2005.) The $1 \mathrm{~m}$ resolution IKONOS stereo imagery is offered by Space Imaging, Inc. (hereinafter called the vendor) to commercial users only as Reference Stereo and Precision Stereo products. They are distributed in an 8 or 11 bit GeoTiff format with an ASCII metadata file (The order parameters of the RFM and the descriptions of the source image and products are included in the metadata file). The description of the several levels of IKONOS products is listed in Table 3-1. The Reference Stereo imagery has a positioning accuracy (RMSE) of $11.8 \mathrm{~m}$ horizontal and $13.4 \mathrm{~m}$ vertical, and the accuracy of the Precision Stereo imagery is $0.9 \mathrm{~m}$ horizontal and $1.8 \mathrm{~m}$ vertical (Grodecki and Dial, 2001). Accuracy can be improved with ground control. Precision and Reference Stereo images refer to stereo images processed respectively with and without ground control. The stereo products include camera model data along with the imagery. The camera model data in the RPC format enables users with suitable software to photogrammetrically process the imagery. Image orientation is described by the RPC data for both Precision and Reference Stereo, the difference being that RPC coefficients for Precision Stereo images have been updated by use of ground control and so are more accurate. Both images of an IKONOS stereo pair are collected on the same orbital pass 
(in-track) as illustrated in Figure 3-1. Typically, one image is collected above $72^{\circ}$ elevation angle and the other above $60^{\circ}$ with a $30-45^{\circ}$ convergence angle between the two images. The convergence angle facilitates three-dimensional measurement (Dial et al., 2003), while the high-elevation leg is suitable for orthorectificaiton. The IKONOS stereo images have a large $\mathrm{B} / \mathrm{H}$ ratioand have a potential of generating DEMs with an accuracy of about $2 \mathrm{~m}$ (Ridley et al., 1997). In fact, same date in-track stereo data acquisition has a strong advantage over multidate across-track stereo data acquisition because it reduces radiometric image variations (temporal changes, sun illumination, etc.), and thus increases the correlation success rate in any image process (Toutin, 2000; 2004).

Table 3-1 IKONOS product levels

\begin{tabular}{|c|c|c|c|c|c|}
\hline & \multicolumn{2}{|c|}{$\begin{array}{c}\text { Positional } \\
\text { Accuracy (m) }\end{array}$} & \multirow{2}{*}{$\begin{array}{l}\text { Target } \\
\text { Elevation } \\
\text { Angle }\left(^{\circ}\right)\end{array}$} & \multirow[t]{2}{*}{$\begin{array}{l}\text { Stereo } \\
\text { Option }\end{array}$} & \multirow[t]{2}{*}{ Applications } \\
\hline & CE90 & RMSE & & & \\
\hline Geo & 15.0 & N/A & $60-90$ & No & $\begin{array}{l}\text { Visual and interpretive } \\
\text { applications }\end{array}$ \\
\hline $\begin{array}{l}\text { Standard } \\
\text { Ortho }\end{array}$ & 50.0 & 25.0 & $60-90$ & No & Basic mapping project \\
\hline Reference & 25.4 & 11.8 & $60-90$ & Yes & $\begin{array}{l}\text { Regional, large area } \\
\text { mapping and general } \\
\text { GIS applications }\end{array}$ \\
\hline Pro & 10.2 & 4.8 & $66-90$ & No & $\begin{array}{l}\text { Transportation, } \\
\text { infrastructure, utilities } \\
\text { planning, economic } \\
\text { development }\end{array}$ \\
\hline Precision & 4.1 & 1.9 & $72-90$ & Yes & $\begin{array}{l}\text { High positional } \\
\text { accuracy for urban } \\
\text { applications }\end{array}$ \\
\hline $\begin{array}{c}\text { Precision } \\
\text { Plus }\end{array}$ & 2.0 & 0.9 & $75-90$ & No & $\begin{array}{l}\text { Detailed urban } \\
\text { analysis, cadastral \& } \\
\text { infrastructure mapping }\end{array}$ \\
\hline
\end{tabular}




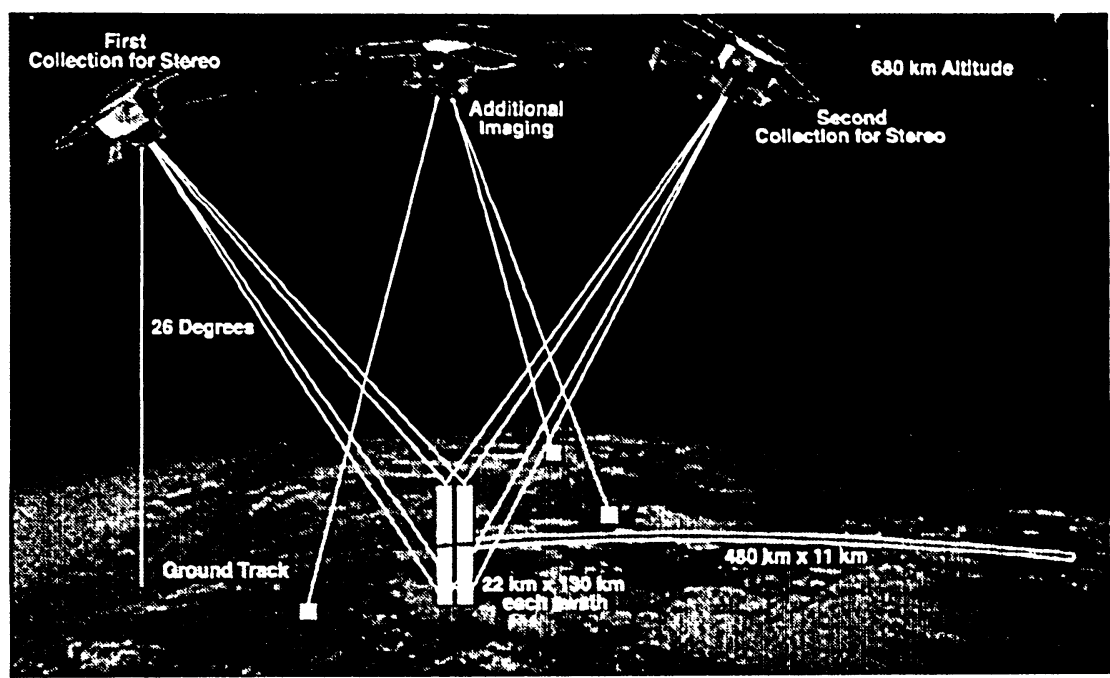

Figure3-2 Same date in-track IKONOS stereo data acquisition (Dial and Grodecki, 2003).

\subsubsection{Study Areas}

The satellite image data used in this study were $1 \mathrm{~m}$ spatial resolution PAN IKONOS stereo images, taken on January 2004, covering Toronto Pearson International Airport and its surrounding area in the GTA, Ontario. The area covered by the IKONOS stereo pair in this study is approximately $11 \mathrm{~km}$ by $13 \mathrm{~km}$ with an elevation range from about 20 $\mathrm{m}$ to $187 \mathrm{~m}$ above the MSL (Datum NAD27). The stereo images are distributed in an 8-bit GeoTiff format with an ASCII metadata file (including order parameters, source image and products file descriptions). However, detailed orbital information is not included. The stereo images are the vender's Reference level products with the Universal Transverse Mercator (UTM) projection, Zone 17 and the WGS 84 Datum. The RPCs in the format of a text file were provided together with the stereo pair. Some 
information regarding the stereo images, including the date, time, pointing angles and sun angels, is listed in Table 3-2. Figure 3-3 is one of the stereo images showing the study area.

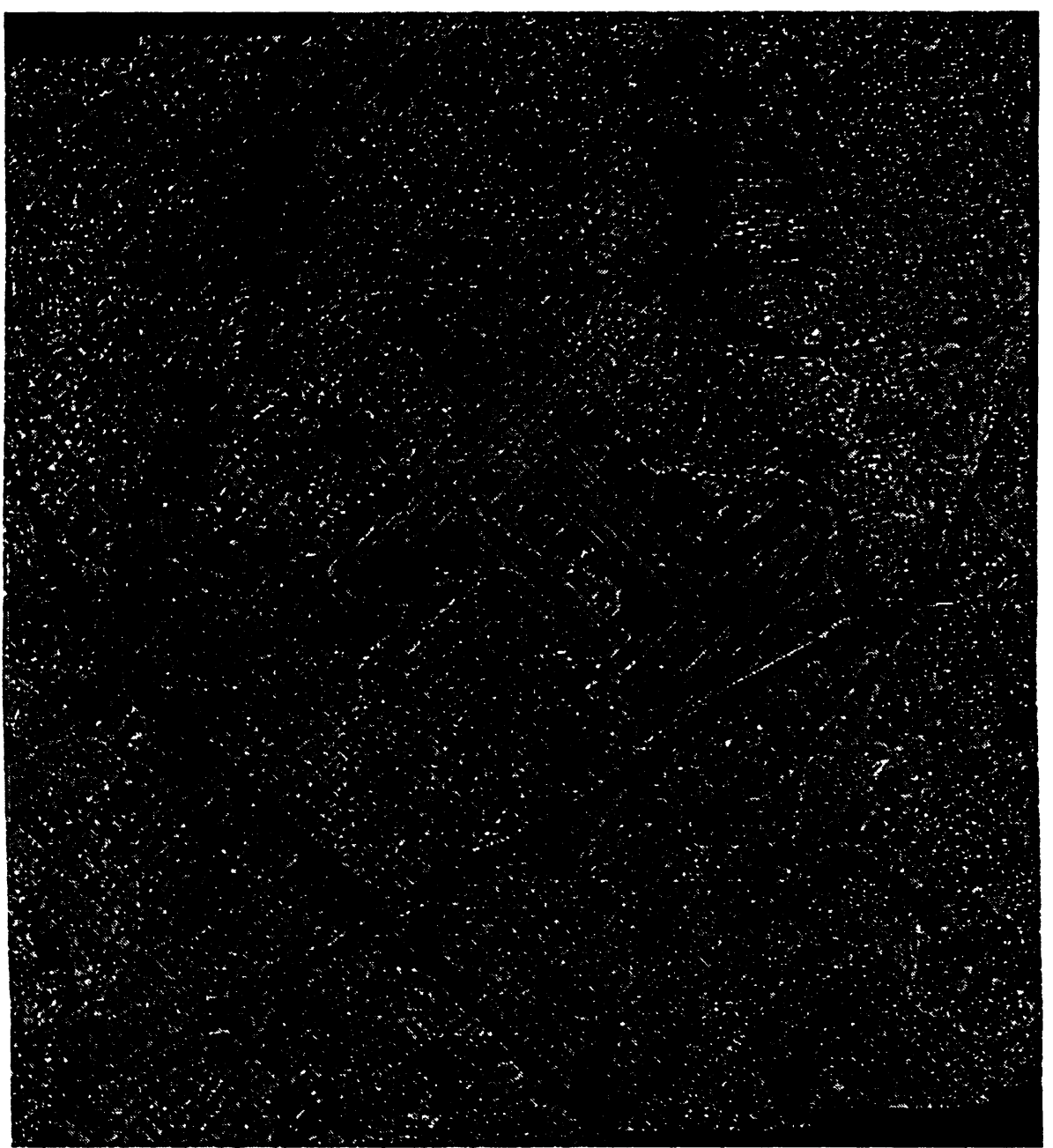

Figure 3-3 Left image of the IKONOS stereo pair acquired on 20 January 2004, north of Toronto, Ontario, Canada. 
Table 3-2 Specifications of the IKONOS stereo Images

\begin{tabular}{|c|c|c|}
\hline Field & Left Image & Right Image \\
\hline $\begin{array}{l}\text { Processing } \\
\text { Level }\end{array}$ & Standard Geometrically Corrected & $\begin{array}{l}\text { Standard } \quad \text { Geometrically } \\
\text { Corrected }\end{array}$ \\
\hline Image Type & PAN & PAN \\
\hline Stereo/Mono & Mono & Mono \\
\hline Datum & WGS84 & WGS84 \\
\hline $\begin{array}{l}\text { Map } \\
\text { Projection }\end{array}$ & UTM & UTM \\
\hline $\begin{array}{l}\text { Zone } \\
\text { Number }\end{array}$ & $17 \mathrm{~N}$ & $17 \mathrm{~N}$ \\
\hline $\begin{array}{l}\text { Collection } \\
\text { Azimuth }\end{array}$ & $37^{\circ} .97$ & $131^{\circ} .53$ \\
\hline $\begin{array}{l}\text { Collection } \\
\text { Elevation }\end{array}$ & $63^{\circ} .58$ & $76^{\circ} .54$ \\
\hline $\begin{array}{l}\text { Acquisition } \\
\text { time }\end{array}$ & 2004-01-20 16:20 GMT & 2004-01-20 16:21 GMT \\
\hline Image & $43^{\circ} .6144942746$ to & $43^{\circ} .6144942746$ to \\
\hline Extension & $\begin{array}{l}43^{\circ} .7332767906 \mathrm{~N} \\
79^{\circ} .5642523475 \text { to } \\
79^{\circ} .7142530565 \mathrm{E}\end{array}$ & $\begin{array}{l}43^{\circ} .7332767906 \mathrm{~N} \\
79^{\circ} .5642523475 \text { to } \\
79^{\circ} .7142530565 \mathrm{E}\end{array}$ \\
\hline
\end{tabular}

\subsection{Strategy for DEM Generation}

As discussed in the previous sections, the vendor does not provide the rigorous sensor model together with IKONOS image data. This causes a major problem in the process of DEM extraction from IKONOS stereo images, i.e., a mathematical model is required to represent the relationship between the $3 \mathrm{D}$ ground coordinates and $2 \mathrm{D}$ image coordinates. 
The processing steps for DEM generation using IKONOS stereo images are similar to that using other types of stereo images, which consist of data collection and preprocessing, GCP collection, mathematical model selection, stereo bundle adjustment with GCPs, elevation parallax measurements, DEM generation and editing, and accuracy evaluation. These seven processing steps (see Figure 3-4) are summarized as follows.

(1) Acquisition and preprocessing of IKONOS stereo data including images and metadata that contain the RPC files, to determine an approximate value for each parameter of the 3D stereo model for the two images.

(2) Collection of GCPs and ICPs with their 3D ground coordinates and 2D image coordinates in the two images, respectively. GCPs should be distributed over the entire region of interest with points at the lowest and highest elevation to avoid extrapolations, both in planimetry and elevation. The image pointing accuracy should be 1-2 pixels for IKONOS. Some points collected in the same way will be used as the ICPs to evaluate the accuracy of the final DEMs.

(3) Selection of the 3D stereo mathematical model that represents the relationship between the $3 \mathrm{D}$ ground coordinates and $2 \mathrm{D}$ image coordinates of the image points. The linearization of some non-linear mathematical models is required before further computation. 
(4) Computation of the $3 \mathrm{D}$ stereo model, initialized with the approximate parameter values and refined by an iterative least-squares bundle adjustment with GCPs. GCPs residuals and ICPs errors are used to indicate the differences between the "true" terrain coordinates and the computed terrain coordinates.

(5) Image matching of the two images to find the conjugate points in the stereo pair by computing the maximum of the correlation coefficient. This method has been commonly used with good results in satellite image matching (Gülch, 1991). After matching, manual editing of the matching results is needed before DEM generation.

(6) Computation of the 3D ground coordinates from the matched points and generation of a regular DEM grid spacing using the previously computed stereo model with a 3D least-squares stereo intersection.

(7) Evaluation of the final DEMs derived from satellite data, through comparing with the DEM generated from different mathematical models, comparing the DEMs extracted with the "true" terrain, and checking the errors of ICPs on the final DEMs.

The above processing steps are similar for all 3D stereo models that are selected. The difference is that some model may not need GCPs, for example, when using the RFM with the RPCs that come with the IKONOS stereo images. 


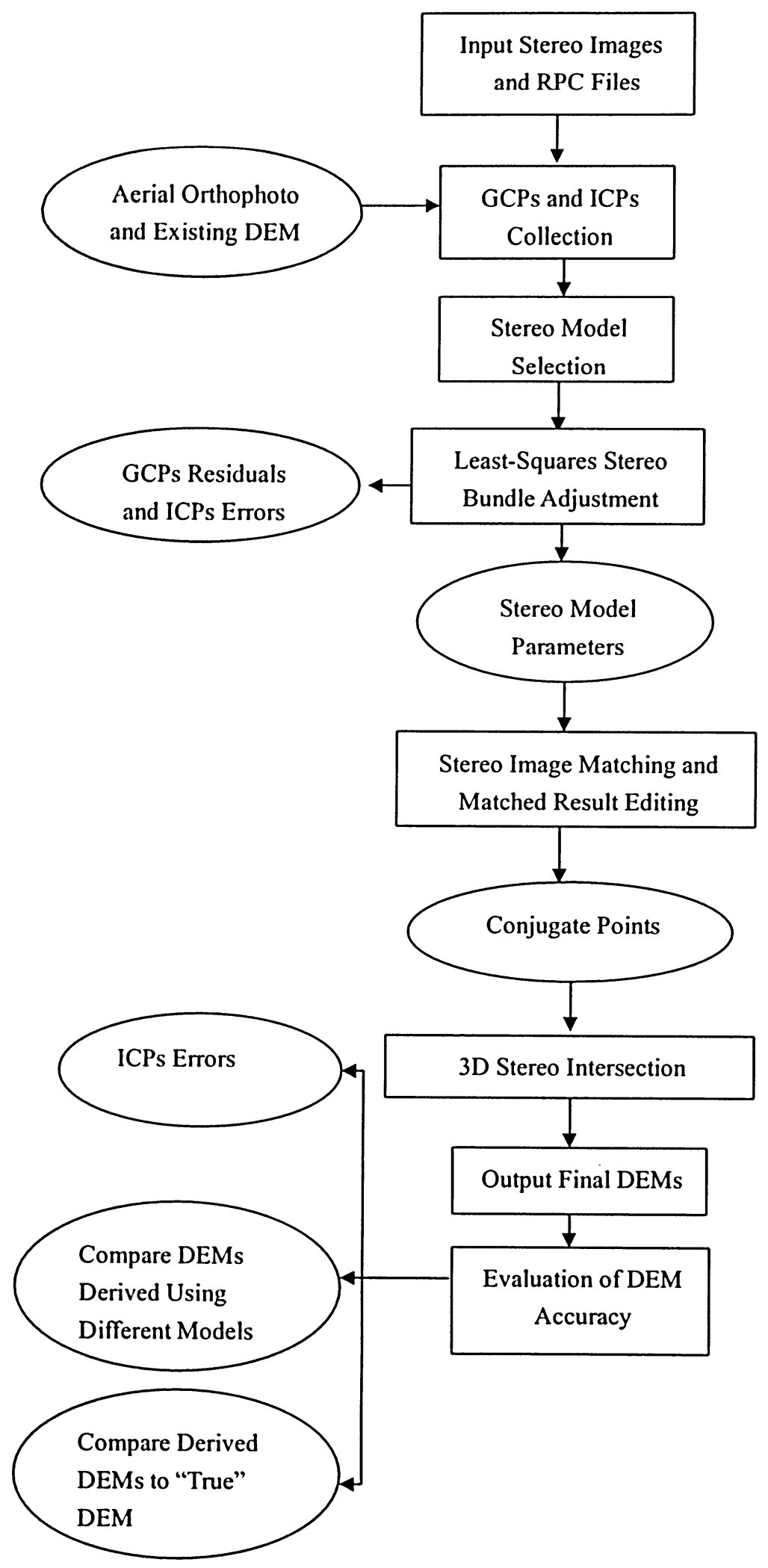

Figure 3-4 Processing steps for the DEM extraction from IKONOS stereo images and its accuracy evaluation. 


\subsection{GCPs Collection}

Photogrammetric control consists of any points whose positions are known in object space or in a ground reference coordinate system and whose images can be positively identified in the photographs. The photogrammetric control, or commonly called ground control, provides the means for orienting or relating photographs to the ground. Photogrammetric control is generally classified as either horizontal control (the planimetric position of the point in object space is known with respect to a horizontal datum), or vertical control (the elevation of the point is known with respect to vertical datum), or both.

High-resolution remote sensing images have to be geometrically and precisely processed with ground control information, such as GCPs to generate accurate map products and 3D geospatial information. Whatever the model used, even the RFM approach to remove the bias or refine RFM parameters, some GCPs have to be acquired to compute/refine the parameters of the mathematical functions in order to achieve a good accuracy. Generally, an iterative least-squares adjustment process is applied when more GCPs than the minimum number required by the 3D stereo model (in comparison to the number of unknown parameters) are used. The number of GCPs is a function of different conditions: the method of collection, the sensor type and resolution, the image spacing, the geometric model, the study site, the physical environment, GCP definition and accuracy and the final expected accuracy (Toutin, 2004). 
A total of 63 points were manually collected in a desktop ERDAS Imagine V8.6 environment from a $0.1 \mathrm{~m}$ pixel spacing orthophoto and the corresponding DEM with $0.5 \mathrm{~m}$ elevation accuracy provided by Spatial Gep-Link, Inc, Markham, Ontario. Points are mainly the end of the sidewalks and some landmarks, which are more easily identifiable in the IKONOS stereo images used. The GCPs are in the UTM projection. Clarke 1866 reference ellipsoid, the elevations of the GCPs is referenced to the Mean Sea Level (MSL), which is different from the WGS84 ellipsoid used for IKONOS imagery. The exact conversion of the projection and elevation values between these two geodetic systems was done using NTv2 and GPS HT2.0 software packages, respectively, provided by the Geodetic Survey Division, Natural Resources Canada website (http://www.geod.nrcan.gc.Ca/index_e/online_apps_e/appNtv2_e/ntv2_geo_utm_e.html, last accessed $23 \mathrm{Feb} 2005)$. NTv2 allows the transformation from the datum NAD27 to NAD83. The GPS HT2.0 allows converting the elevation from above MSL to the elevation above the ellipsoid.

\subsection{Sensor Models of IKONOS Imagery}

A sensor model expresses the relationship between the 3D object point positions and their corresponding 2D image positions. It geometrically relates the image space and the object space. The physical sensor models and generalized sensor models are two broad categories of sensor models that are used (McGlone, 1996). A well-designed sensor model ensures that the products generated from the imagery are accurate. Unfortunately, the IKONOS vendor does not provide any physical model. Two sensor models have 
been used to process IKONOS stereo images: the RFM and the 3D physical model developed at the CCRS. The description and calculation of these two models are detailed in the following sections.

\subsubsection{Rational Function Model}

The RFM is one of the image transfer standards for earth image as well as the polynomial model, grid interpolation model, universal real-time image geometry model which are recommended by the Open Geospatial Consortium (OGC). It has been used as a replacement sensor model in many commercial photogrmmetric systems (Greve et al., 1989; Madani, 1999; Dowman and Dolloff, 2000). The feasibility of using the RFM to replace the physical sensor models has been investigated by many researchers using different types of imagery.

\section{Definition of the RFM}

In the RFM, image pixel coordinates $(x, y)$ are expressed as the ratios of polynomials of ground coordinates in the latitude $(\mathrm{P})$, longitude $(\mathrm{L})$, and elevation $(\mathrm{H})$, i.e., $(\mathrm{P}, \mathrm{L}, \mathrm{H})$. In order to minimize the errors introduced during the computations and improve the numerical stability of equations, the image and ground coordinates are each offset and scaled to fit within the range of -1.0 to +1.0 (NIMA, 2000). The transformation from the ground coordinates to the image coordinates can be defined as: 


$$
\begin{gathered}
x=\frac{\sum_{i=1}^{20} S A M P_{i}-N U M \_C O E F_{i} \cdot \rho_{i}(P, L, H)}{\sum_{i=1}^{20} S A M P_{-} D E N_{-} C O E F_{i} \cdot \rho_{i}(P, L, H)} \\
y=\frac{\sum_{i=1}^{20} L I N E \_N U M \_C O E F_{i} \cdot \rho_{i}(P, L, H)}{\sum_{i=1}^{20} L I N E \_D E N \_C O E F_{i} \cdot \rho_{i}(P, L, H)}
\end{gathered}
$$

where

$x$ and $y$ are the normalized image coordinates;

$P$ is the normalized latitude;

$L$ is the normalized longitude;

$H$ is the normalized height;

and the definition of the polynomials in the numerator and denominator are defined as following:

$$
\begin{gathered}
\sum_{i=1}^{20} C_{i} \cdot \rho_{i}(P, L, H)=C_{1}+C_{2} \cdot L+C_{3} \cdot P+C_{4} \cdot H+C_{5} \cdot L \cdot P+C_{6} \cdot L \cdot H+C_{7} \cdot P \cdot H+ \\
C_{8} \cdot L^{2}+C_{9} \cdot P^{2}+C_{10} \cdot H^{2}+C_{11} \cdot P \cdot L \cdot H+C_{12} \cdot L^{3}+C_{13} \cdot L \cdot P^{2}+C_{14} \cdot L \cdot H^{2} \\
C_{15} \cdot L^{2} \cdot P+C_{16} \cdot P^{3}+C_{17} \cdot P \cdot H^{2}+C_{18} \cdot L^{2} \cdot H+C_{19} \cdot P^{2} \cdot H+C_{20} \cdot H^{3}
\end{gathered}
$$

The parameter meanings of IKONOS stereo imagery provided by the vendor in the format of "image_name_rpc.txt" are described as follows:

LINE_OFF; $\quad$ Line Offset

SAMP_OFF: Sample Offset

LAT_OFF: $\quad$ Latitude Offset 
LONG_OFF:

HEIGHT_OFF:

LINE_SCALE:

SAMP_SCALE:

LAT_SCALE:

LONG_SCALE:

HEIGHT_SCALE:

LINE_NUM_COEF_1 to 20: $\quad 20$ Line Numerator Coefficients

LINE_DEN_COEF_1 to 20: 20 Line Denominator Coefficients

SAMP_NUM_COEF_1 to 20: 20 Line Numerator Coefficients

SAMP_DEN_COEF_1 to 20: 20 Line Denominator Coefficients

With the knowledge of Latitude, Longitude, and Height, the process of converting latitude, longitude, and height to normalized units could be done using the following equations:

$$
\begin{aligned}
& P=\frac{\text { Latitude }-L A T \_O F F}{L A T-S C A L E} \\
& L=\frac{\text { Longitude }- \text { LONG_OFF }}{\text { LONG_SCALE }} \\
& H=\frac{\text { Height }-H E I G H T-O F F}{H E I G H T-S C A L E}
\end{aligned}
$$

where,

Latitude is the geodetic latitude expressed in degrees in the product order datum; 
Longitude is the geodetic longitude expressed in degrees; and

Height is the geodetic height expressed in metres above the WGS84 reference ellipsoid.

Finally, the $(x, y)$ coordinates of the line and sample need to be de-normalized using the following equations:

$$
\begin{aligned}
& \text { Row }=y \cdot L I N E \_ \text {SCALE }+ \text { LINE_OFF } \\
& \text { Col }=x \cdot S A M P P_{-} S C A L E+S A M P{ }_{-} O F F
\end{aligned}
$$

where

Row is the image line number expressed in pixels with zero as the centre of the first line.

$\mathrm{Col}$ is the sample number expressed in pixels with zero is the centre of the left-most sample.

The RFM is essentially a generic form of polynomial when the denominator is equal to 1, Equations 3-1 and 3-2 become regular 3D polynomials. Using the RPCs, the distortions caused by the optical projection can be generally represented by ratios of the $1^{\text {st }}$-order terms, while corrections such as the Earth's curvature, atmospheric refraction, and lens distortion, etc., can be well approximated by the $2^{\text {nd }}$-order terms. Some other distortions with higher-order components can be modeled using the $3^{\text {rd }}$-order terms in the RFM (Tao and Hu, 2001). 
The RPCs can be directly used for the transformation from object to pixel coordinates. However, the transformation from pixel to object coordinates is the inverse procedure and needs an iterative calculation due to the non-linearity of the RPCs. With the vendor-supplied RPCs, there are $3^{\text {rd }}$-order polynomials and RPC has 80 terms polynomials and 10 terms of offset and scale factor as mentioned above. Practically, the two polynomials used in denominator are identical, while the first coefficient of the denominator is 1 , to avoid the case when the denominator becomes close to zero, this thus results in 59 parameters remaining.

\section{RFM-based 3D Reconstruction Method}

According to the above description in Equations 3-1 and 3-2, the relationships of (Latitude, Longitude, and Height) position $(P, L, H)$ and (Row, Col) coordinate $(x, y)$ can be described as following and the definition of coordinates of the conjugate points $(x$, $\left.y^{\prime}\right)$ has the similar definition:

$$
\begin{gathered}
x=\frac{\sum_{i=1}^{20} a_{i} \cdot \rho_{i}(P, L, H)}{\sum_{i=1}^{20} b_{i} \cdot \rho_{i}(P, L, H)} \\
y=\frac{\sum_{i=1}^{20} c_{i} \cdot \rho_{i}(P, L, H)}{\sum_{i=1}^{20} d_{i} \cdot \rho_{i}(P, L, H)}
\end{gathered}
$$

where

$$
a_{i}, b_{i}, c_{i}, d_{i} \text { are the polynomial coefficients provided by the vendor, } \rho_{i} \text { are }
$$


polynomial functions with the following coefficients:

$$
\begin{array}{llll}
\rho_{1}(P, L, H)=1 & & \\
\rho_{2}(P, L, H)=L, & \rho_{3}(P, L, H)=P, & \rho_{4}(P, L, H)=H & \\
\rho_{5}(P, L, H)=L P, & \rho_{6}(P, L, H)=L H, & \rho_{7}(P, L, H)=P H & \\
\rho_{8}(P, L, H)=L^{2}, & \rho_{9}(P, L, H)=P^{2}, & \rho_{10}(P, L, H)=H^{2} & \\
\rho_{11}(P, L, H)=P L H, & \rho_{12}(P, L, H)=L^{3}, & \rho_{13}(P, L, H)=L P^{2}, & \rho_{14}(P, L, H)=L H^{2} \\
\rho_{15}(P, L, H)=L^{2} P, & \rho_{16}(P, L, H)=P^{3}, & \rho_{17}(P, L, H)=P H^{2}, & \rho_{18}(P, L, H)=L^{2} H \\
\rho_{19}(P, L, H)=P^{2} H, & \rho_{20}(P, L, H)=H^{3}, & &
\end{array}
$$

From the coordinates $(x, y)$ and $\left(x^{\prime}, y^{\prime}\right)$ of the conjugate image points on the stereo pair respectively, the following four equations can be derived:

$$
\begin{aligned}
& F_{x}=\sum_{i=1}^{20} a_{i} \rho_{i}(P, L, H)-x \sum_{i=1}^{20} b_{i} \rho_{i}(P, L, H)=0 \\
& F_{y}=\sum_{i=1}^{20} c_{i} \rho_{i}(P, L, H)-y \sum_{i=1}^{20} d_{i} \rho_{i}(P, L, H)=0 \\
& F_{x}=\sum_{i=1}^{20} a^{\prime}{ }_{i} \rho_{i}(P, L, H)-x \sum_{i=1}^{20} b_{i}^{\prime} \rho_{i}(P, L, H)=0 \\
& F_{y}=\sum_{i=1}^{20} c^{\prime}{ }_{i} \rho_{i}(P, L, H)-y^{\prime} \sum_{i=1}^{20} d^{\prime}{ }_{i} \rho_{i}(P, L, H)=0
\end{aligned}
$$

The following equation can be derived by linearizing Equation 3-7,

$$
\begin{aligned}
& \frac{\partial F_{x}}{\partial P} \Delta P+\frac{\partial F_{x}}{\partial L} \Delta L+\frac{\partial F_{x}}{\partial H} \Delta H+F_{x}\left(P_{0}, L_{0}, H_{0}\right)=0 \\
& \frac{\partial F_{y}}{\partial P} \Delta P+\frac{\partial F_{y}}{\partial L} \Delta L+\frac{\partial F_{y}}{\partial H} \Delta H+F_{y}\left(P_{0}, L_{0}, H_{0}\right)=0 \\
& \frac{\partial F_{x}}{\partial P} \Delta P+\frac{\partial F_{x}}{\partial L} \Delta L+\frac{\partial F_{x}}{\partial H} \Delta H+F_{x}\left(P_{0}, L_{0}, H_{0}\right)=0 \\
& \frac{\partial F_{y}}{\partial P} \Delta P+\frac{\partial F_{y}}{\partial L} \Delta L+\frac{\partial F_{y}}{\partial H} \Delta H+F_{y}\left(P_{0}, L_{0}, H_{0}\right)=0
\end{aligned}
$$


And the error equation of Equation 3-8 can be described in a matrix format:

$$
\mathrm{V}=\mathrm{AX}-\mathrm{L}
$$

where,

$$
\begin{gathered}
\mathrm{A}=\left[\begin{array}{lll}
\frac{\partial F_{x}}{\partial P} & \frac{\partial F_{x}}{\partial L} & \frac{\partial F_{x}}{\partial H} \\
\frac{\partial F_{y}}{\partial P} & \frac{\partial F_{y}}{\partial L} & \frac{\partial F_{y}}{\partial H_{y}} \\
\frac{\partial F_{x}}{\partial P} & \frac{\partial F_{x}}{\partial L} & \frac{\partial F_{x}}{\partial H} \\
\frac{\partial F_{y}}{\partial P} & \frac{\partial F_{y}}{\partial L} & \frac{\partial F_{y}}{\partial H}
\end{array}\right] \\
\mathrm{X}=\left[\begin{array}{l}
\Delta P \\
\Delta L \\
\Delta H
\end{array}\right] \\
\mathrm{L}=\left[\begin{array}{l}
-F_{x}\left(P_{0}, L_{0}, H_{0}\right) \\
-F_{y}\left(P_{0}, L_{0}, H_{0}\right) \\
-F_{x}\left(P_{0}, L_{0}, H_{0}\right) \\
-F_{y}\left(P_{0}, L_{0}, H_{0}\right)
\end{array}\right]
\end{gathered}
$$

where 


$$
\begin{aligned}
& \frac{\partial F_{x}(P, L, H)}{\partial P}=\sum_{i}\left(a_{i}-x b_{i}\right) \frac{\partial \rho_{i}(P, L, H)}{\partial P}, i=3,5,7,9,11,13,15,16,17,19 \\
& \frac{\partial F_{x}(P, L, H)}{\partial L}=\sum_{i}\left(a_{i}-x b_{i}\right) \frac{\partial \rho_{i}(P, L, H)}{\partial L}, i=2,5,6,8,11,12,13,14,15,18 \\
& \frac{\partial F_{x}(P, L, H)}{\partial H}=\sum_{i}\left(a_{i}-x b_{i}\right) \frac{\partial \rho_{i}(P, L, H)}{\partial H}, i=4,6,7,10,11,14,17,18,19,20 \\
& \frac{\partial F_{y}(P, L, H)}{\partial P}=\sum_{i}\left(c_{i}-y d_{i}\right) \frac{\partial \rho_{i}(P, L, H)}{\partial P}, i=3,5,7,9,11,13,15,16,17,19 \\
& \frac{\partial F_{y}(P, L, H)}{\partial L}=\sum_{i}\left(c_{i}-y d_{i}\right) \frac{\partial \rho_{i}(P, L, H)}{\partial L}, i=2,5,6,8,11,12,13,14,15,18 \\
& \frac{\partial F_{y}(P, L, H)}{\partial H}=\sum_{i}\left(c_{i}-y d_{i}\right) \frac{\partial \rho_{i}(P, L, H)}{\partial H}, i=4,6,7,10,11,14,17,18,19,20 \\
& \frac{\partial F_{x^{\prime}}(P, L, H)}{\partial P}=\sum_{i}\left(a_{i}^{\prime}-x^{\prime} b_{i}^{\prime}\right) \frac{\partial \rho_{i}(P, L, H)}{\partial P}, i=3,5,7,9,11,13,15,16,17,19 \\
& \frac{\partial F_{x^{\prime}}(P, L, H)}{\partial L}=\sum_{i}\left(a_{i}{ }_{i}-x^{\prime} b_{i}^{\prime}\right) \frac{\partial \rho_{i}(P, L, H)}{\partial L}, i=2,5,6,8,11,12,13,14,15,18 \\
& \frac{\partial F_{\dot{x}}(P, L, H)}{\partial H}=\sum_{i}\left(a_{i}{ }_{i}-x^{\prime} b_{i}^{\prime}\right) \frac{\partial \rho_{i}(P, L, H)}{\partial H}, i=4,6,7,10,11,14,17,18,19,20 \\
& \frac{\partial F_{y^{\prime}}(P, L, H)}{\partial P}=\sum_{i}\left(c_{i}{ }_{i}-y^{\prime} d^{\prime}{ }_{i}\right) \frac{\partial \rho_{i}(P, L, H)}{\partial P}, i=3,5,7,9,11,13,15,16,17,19 \\
& \frac{\partial F_{y}(P, L, H)}{\partial L}=\sum_{i}\left(c^{{ }^{\prime}}{ }_{i}-y^{\prime} d^{\prime}{ }_{i}\right) \frac{\partial \rho_{i}(P, L, H)}{\partial L}, i=2,5,6,8,11,12,13,14,15,18 \\
& \frac{\partial F_{y}(P, L, H)}{\partial H}=\sum_{i}\left(\dot{c}_{i}-y^{\prime} d_{i}^{\prime}\right) \frac{\partial \rho_{i}(P, L, H)}{\partial H}, i=4,6,7,10,11,14,17,18,19,20
\end{aligned}
$$

The first-order derivatives of polynomial $\rho_{1}$ are:

$$
\frac{\partial \rho_{1}(P, L, H)}{\partial P}=\frac{\partial \rho_{1}(P, L, H)}{\partial L}=\frac{\partial \rho_{1}(P, L, H)}{\partial H}=0
$$

The first-order derivatives of polynomials $\rho_{i}(i=2, \ldots, 20)$ for $\mathrm{P}$ are: 


$$
\begin{array}{llll}
\frac{\partial \rho_{2}(P, L, H)}{\partial P}=0 & \frac{\partial \rho_{3}(P, L, H)}{\partial P}=1 & \frac{\partial \rho_{4}(P, L, H)}{\partial P}=0 \\
\frac{\partial \rho_{5}(P, L, H)}{\partial P}=L & \frac{\partial \rho_{6}(P, L, H)}{\partial P}=0 & \frac{\partial \rho_{7}(P, L, H)}{\partial P}=H & \\
\frac{\partial \rho_{8}(P, L, H)}{\partial P}=0 & \frac{\partial \rho_{9}(P, L, H)}{\partial P}=2 P & \frac{\partial \rho_{10}(P, L, H)}{\partial P}=0 & \\
\frac{\partial \rho_{11}(P, L, H)}{\partial P}=L H & \frac{\partial \rho_{12}(P, L, H)}{\partial P}=0 & \frac{\partial \rho_{13}(P, L, H)}{\partial P}=2 L P & \frac{\partial \rho_{14}(P, L, H)}{\partial P}=0 \\
\frac{\partial \rho_{15}(P, L, H)}{\partial P}=L^{2} & \frac{\partial \rho_{16}(P, L, H)}{\partial P}=3 P^{2} & \frac{\partial \rho_{17}(P, L, H)}{\partial P}=H^{2} & \frac{\partial \rho_{18}(P, L, H)}{\partial P}=0 \\
\frac{\partial \rho_{19}(P, L, H)}{\partial P}=2 P H & \frac{\partial \rho_{20}(P, L, H)}{\partial P}=0 & &
\end{array}
$$

The first-order derivatives of polynomials $\rho_{i}(i=2, \ldots, 20)$ for $\mathrm{L}$ are:

$$
\begin{array}{llll}
\frac{\partial \rho_{2}(P, L, H)}{\partial L}=1 & \frac{\partial \rho_{3}(P, L, H)}{\partial L}=0 & \frac{\partial \rho_{4}(P, L, H)}{\partial L}=0 \\
\frac{\partial \rho_{5}(P, L, H)}{\partial L}=P & \frac{\partial \rho_{6}(P, L, H)}{\partial L}=H & \frac{\partial \rho_{7}(P, L, H)}{\partial L}=0 & \\
\frac{\partial \rho_{8}(P, L, H)}{\partial L}=2 L & \frac{\partial \rho_{9}(P, L, H)}{\partial L}=0 & \frac{\partial \rho_{10}(P, L, H)}{\partial L}=0 & \\
\frac{\partial \rho_{11}(P, L, H)}{\partial L}=P H & \frac{\partial \rho_{12}(P, L, H)}{\partial L}=3 L^{2} & \frac{\partial \rho_{13}(P, L, H)}{\partial L}=P^{2} & \frac{\partial \rho_{14}(P, L, H)}{\partial L}=H^{2} \\
\frac{\partial \rho_{15}(P, L, H)}{\partial L}=2 L P & \frac{\partial \rho_{16}(P, L, H)}{\partial L}=0 & \frac{\partial \rho_{17}(P, L, H)}{\partial L}=0 & \frac{\partial \rho_{18}(P, L, H)}{\partial L}=2 L H \\
\frac{\partial \rho_{19}(P, L, H)}{\partial L}=0 & \frac{\partial \rho_{20}(P, L, H)}{\partial L}=0 & &
\end{array}
$$

The first-order derivatives of polynomials $\rho_{i}(i=2, \ldots, 20)$ for $\mathrm{H}$ are: 


$$
\begin{array}{llll}
\frac{\partial \rho_{2}(P, L, H)}{\partial H}=0 & \frac{\partial \rho_{3}(P, L, H)}{\partial H}=0 & \frac{\partial \rho_{4}(P, L, H)}{\partial H}=1 \\
\frac{\partial \rho_{5}(P, L, H)}{\partial H}=0 & \frac{\partial \rho_{6}(P, L, H)}{\partial H}=L & \frac{\partial \rho_{7}(P, L, H)}{\partial H}=P \\
\frac{\partial \rho_{8}(P, L, H)}{\partial H}=0 & \frac{\partial \rho_{9}(P, L, H)}{\partial H}=0 & \frac{\partial \rho_{10}(P, L, H)}{\partial H}=2 H & \\
\frac{\partial \rho_{11}(P, L, H)}{\partial H}=P L & \frac{\partial \rho_{12}(P, L, H)}{\partial H}=0 & \frac{\partial \rho_{13}(P, L, H)}{\partial H}=0 & \frac{\partial \rho_{14}(P, L, H)}{\partial H}=2 L H \\
\frac{\partial \rho_{15}(P, L, H)}{\partial H}=0 & \frac{\partial \rho_{16}(P, L, H)}{\partial H}=0 & \frac{\partial \rho_{17}(P, L, H)}{\partial H}=2 P H & \frac{\partial \rho_{18}(P, L, H)}{\partial H}=L^{2} \\
\frac{\partial \rho_{19}(P, L, H)}{\partial H}=P^{2} & \frac{\partial \rho_{20}(P, L, H)}{\partial H}=3 H^{2} &
\end{array}
$$

By giving the initial values $\left(P_{0}, L_{0}, H_{0}\right)$ of $(P, L, H)$, the constant terms in Equation 3-8 can be expressed as:

$$
\begin{aligned}
& F_{x}\left(P_{0}, L_{0}, H_{0}\right)=\sum_{i=1}^{20} a_{i} \rho_{i}\left(P_{0}, L_{0}, H_{0}\right)-x \sum_{i=1}^{20} b_{i} \rho_{i}\left(P_{0}, L_{0}, H_{0}\right)=0 \\
& F_{y}\left(P_{0}, L_{0}, H_{0}\right)=\sum_{i=1}^{20} c_{i} \rho_{i}\left(P_{0}, L_{0}, H_{0}\right)-y \sum_{i=1}^{20} d_{i} \rho_{i}\left(P_{0}, L_{0}, H_{0}\right)=0 \\
& F_{x} \cdot\left(P_{0}, L_{0}, H_{0}\right)=\sum_{i=1}^{20} a_{i} \rho_{i}\left(P_{0}, L_{0}, H_{0}\right)-x \sum_{i=1}^{20} b_{i}^{\prime} \rho_{i}\left(P_{0}, L_{0}, H_{0}\right)=0 \\
& F_{y} \cdot\left(P_{0}, L_{0}, H_{0}\right)=\sum_{i=1}^{20} c_{i} \rho_{i}\left(P_{0}, L_{0}, H_{0}\right)-y \cdot \sum_{i=1}^{20} d^{\prime} \rho_{i}\left(P_{0}, L_{0}, H_{0}\right)=0
\end{aligned}
$$

Using the least-squares methods and solving the results literately until the difference between the nearly two consequent calculations are smaller than a threshold:

$$
\begin{aligned}
& P_{k+1}=P_{k}+\Delta P_{k} \\
& L_{k+1}=L_{k}+\Delta L_{k} \\
& H_{k+1}=H_{k}+\Delta H_{k}
\end{aligned} \quad k=0,1,2, \ldots
$$


To determine the initial approximate values of the object point coordinates $\left(P_{0}, L_{0}, H_{0}\right)$, two main methods could be useful. One method for obtaining these initial values is to use the information in the metadata, which includes the planimetric coordinates of the four corner image points. When the corresponding image coordinates of these four points are known, , the approximate planimetric coordinates of each point can be solved by solving a simple polynomial function. The HEIGHT_OFF provided in the RPC text file could be used as the approximate elevation value. Another method to get these initial coordinates is to solve the RFM, using only the first-order terms and omitting the second and third- $2^{\text {nd }}$ terms. The constant and first-order terms in both the numerator and the denominator in the RPC text file of IKONOS stereo imagery are larger by many orders of magnitude than those of the second and third-order terms. Therefore, the method mentioned above is suitable for the IKONOS stereo imagery.

\section{Improving the 3D Geopositioning Accuracy using GCPs}

The method above can be used in the case when GCPs is not available. The RFM- based 3D reconstruction has to be fulfilled using the vendor supplied RPC file. The position accuracy of the Reference stereo product of Space Imaging, which is used in this study, is reported to be $25.4 \mathrm{~m} \mathrm{CE90} \mathrm{and} 11.8 \mathrm{~m}$ RMSE, and those of IKONOS Precision and Precision Plus products are $1.9 \mathrm{~m}$ and $0.9 \mathrm{~m}$, respectively. It is expected in many cases to enhance the ground accuracy of the Reference product to the level of Precision or Precision Plus products. 
When end users have access to some ground control for the study area, the 3D reconstruction results achieved using the above method could be improved to Precision or even the Precision Plus level. There are two methods to improve the geopositioning accuracy of the IKONOS Reference product. The first one is to use the vendor-supplied RPCs as the initial values for recalculating the RPCs. This method requires a large number of GCPs to calculate the new RPCs. Actually, more than 39 GCPs are required for the $3^{\text {rd }}$-order RFM. The second method is the bias compensation method, that can improves the accuracy of the ground coordinates derived from the vendor-supplied RPCs using a polynomial correction whose parameters are calculated by the GCPs. This correction could be used to either the image coordinates or the ground coordinates after the $3 \mathrm{D}$ reconstruction, or it could be done by changing the RPCs directly.

\section{Recalculation of the RPCs using GCPs}

This method aims to improve the RPCs that describe the perspective imaging geometry and then enhances the quality of the 3D reconstructions. With the knowledge of the GCPs, the RPCs could be recalculated by using the vendor-supplied RPCs in the iterative least-squares solution. In this case, the solution will highly dependent on the actual terrain relief, the accuracy, number and distribution of GCPs across the scene. The equations used to recalculate the RPCs are described in Equation (3-17).

In this case, the linearized format of Equation 3-7 is given as follows: 


$$
\begin{aligned}
& \sum_{i=1}^{20} \frac{\partial F_{x}}{\partial a_{i}} \Delta a_{i}+\sum_{i=1}^{20} \frac{\partial F_{x}}{\partial b_{i}} \Delta b_{i}+F_{x}\left(a_{1}^{0}, a_{2}^{0}, \ldots a_{20}^{0} b_{2}^{0}, b_{3}^{0}, \ldots b_{20}^{0}\right)=0 \\
& \sum_{i=1}^{20} \frac{\partial F_{y}}{\partial c_{i}} \Delta c_{i}+\sum_{i=1}^{20} \frac{\partial F_{y}}{\partial d_{i}} \Delta d_{i}+F_{y}\left(c_{1}^{0}, c_{2}^{0}, \ldots c_{20}^{0} d_{2}^{0}, d_{3}^{0}, \ldots d_{20}^{0}\right)=0 \\
& \sum_{i=1}^{20} \frac{\partial F_{x^{\prime}}}{\partial a_{i}^{\prime}} \Delta a_{i}^{\prime}+\sum_{i=1}^{20} \frac{\partial F_{x^{\prime}}}{\partial b_{i}^{\prime}} \Delta b_{i}^{\cdot}+F_{x^{\prime}}\left(a_{1}^{.0}, a_{2}^{.0}, \ldots a_{20,}^{.0} b_{2}^{.0}, b_{3}^{.0}, \ldots b_{20}^{.0}\right)=0
\end{aligned}
$$

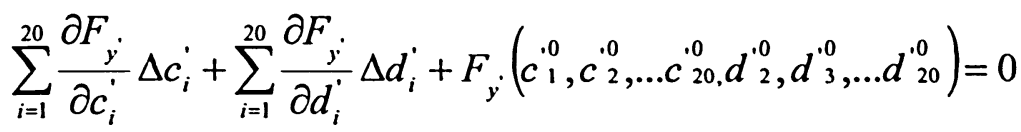

where

$$
\begin{array}{ll}
\sum_{i=1}^{20} \frac{\partial F_{x}}{\partial a_{i}}=\sum_{i=1}^{20} \rho_{i}(P, L, H) & \sum_{i=1}^{20} \frac{\partial F_{x}}{\partial b_{i}} \Delta b_{i}=-x \sum_{i=1}^{20} \rho_{i}(P, L, H) \\
\sum_{i=1}^{20} \frac{\partial F_{y}}{\partial c_{i}}=\sum_{i=1}^{20} \rho_{i}(P, L, H) & \sum_{i=1}^{20} \frac{\partial F_{y}}{\partial d_{i}} \Delta d_{i}=-y \sum_{i=1}^{20} \rho_{i}(P, L, H) \\
\sum_{i=1}^{20} \frac{\partial F_{x^{\prime}}}{\partial a_{i}^{\prime}}=\sum_{i=1}^{20} \rho_{i}(P, L, H) & \sum_{i=1}^{20} \frac{\partial F_{x^{\prime}}}{\partial b_{i}^{\prime}} \Delta b_{i}^{\prime}=-x \sum_{i=1}^{20} \rho_{i}(P, L, H) \\
\sum_{i=1}^{20} \frac{\partial F_{y^{\prime}}}{\partial c_{i}^{\prime}}=\sum_{i=1}^{20} \rho_{i}(P, L, H) & \sum_{i=1}^{20} \frac{\partial F_{y^{\prime}}}{\partial d_{i}^{\prime}} \Delta d_{i}^{\prime}=-y \sum_{i=1}^{20} \rho_{i}(P, L, H)
\end{array}
$$

The next step is to give the initial values of the RPCs using the vender-supplied RPCs. And then to literately calculate the RPCs. The calculation terminates when the absolute difference of the residuals between the two consecutive iterations is below a defined threshold. In practice, it is found that although the vender-supplied coefficients are used as the initial values, the calculation of the new set of RPCs is still not stable when calculating the coefficients in the numerator and denominator at the same time. It turns out that the calculated values of the RPCs are much larger than the vendor-supplied RPCs and the iteration calculation is not stable neither. To solve this problem, the 
coefficients of the numerator or the denominator are fixed first by using the vendor-supplied values to calculate the coefficients in the other part. Then the unknowns in the previous fixed part are calculated. In this way, the calculation is stable and the generated coefficients are similar to the vendor-supplied one. The most possible reason for the unstable calculation may be the high correlation between the coefficients in the numerator and the denominator.

\section{Bias Compensation in the RFM}

The IKONOS imaging system has a narrow angle field of view. This narrow field of view results in almost complete correlation of parameters dealing with satellite position and attitude. Grodecki and Dial (2002) demonstrated that many effects during the generation of the high resolution imagery are negligible or completely correlated with other effects. As a result, only a few parameters are required to effectively model the sensor errors. A line offset parameter is required to adjust for errors in the line direction and a sample offset parameter is required to adjust for errors in the sample direction. The line parameter absorbs effects of orbit, attitude, and residual interior orientation errors in the line direction. The sample parameter absorbs the same effects in the sample direction. For the longer strips, a parameter proportional to line can be added to model drift errors. There are mainly two ways to do the bias compensation, one is in the image domain and the other is in the ground domain. The mathematical models of these two methods are given below: 
The bias compensation model defined in the image domain is defined as:

$$
\begin{aligned}
& \Delta x=a_{0}+a_{s} \cdot \text { Sample }+a_{L} \cdot \text { Line } \\
& \Delta y=b_{0}+b_{s} \cdot \text { Sample }+b_{L} \cdot \text { Line }
\end{aligned}
$$

where

Line and Sample are measured line and sample coordinates of the GCPs;

$\Delta x$ and $\Delta y$ are the adjustable functions expressing the differences between the measured and the nominal line and sample coordinates of the GCPs; $a_{0}, a_{S}, a_{L}$ and $b_{0}, b_{s}, b_{L}$ are the adjustment parameters for an image.

Each of the parameters in Equation 3-19 has a physical significance. Parameter $a_{0}$ absorbs all in-track effects causing offsets in the line direction including in-track ephemeris, satellite pitch attitude, cross-array principal point and cross-array detector positions. For the narrow field of view satellite instruments, all of these physical parameters have the same net effect of displacing images in line. Similarly, parameter $b_{0}$ absorbs all across-track effects causing offsets in the sample direction including across-track ephemeris, satellite roll attitude, along-array principal point and along-array detector positions. Because the line direction is equivalent to time, parameters $a_{L}$ and $b_{L}$ absorb the small effects due to gyro drift during the imaging scan (Dial and Grodecki, 2002). Parameters $a_{s}$ and $b_{s}$ absorb radial ephemeris errors, and interior orientation errors such as focal length and lens distortion errors (Grodecki 
and Dial, 2003).

The ability to determine the bias parameters $\Delta x$ and $\Delta y$ is very useful. But for the use of a softcopy photogrammetry workstation, it would be much easier if the bias compensation could be incorporated into the vendor-supplied RPCs. This would allow bias-free application of RPC-positioning without any reference to additional correction terms. Fraser and Hanley (2003) developed a method to generate bias-corrected RPCs.

$$
\begin{aligned}
& x+\Delta x=\frac{\rho_{1}(P, L, H)}{\rho_{2}(P, L, H)} \\
& y+\Delta y=\frac{\rho_{3}(P, L, H)}{\rho_{4}(P, L, H)}
\end{aligned}
$$

or

$$
\begin{aligned}
& x=\frac{\rho^{c_{1}}(P, L, H)}{\rho_{2}(P, L, H)} \\
& y=\frac{\rho^{c_{3}}(P, L, H)}{\rho_{4}(P, L, H)}
\end{aligned}
$$

where 


$$
\begin{aligned}
& \rho_{1}^{c}(P, L, H)=\left(a_{1}-b_{1} \Delta x\right)+\left(a_{2}-b_{2} \Delta x\right) \cdot P+\left(a_{3}-b_{3} \Delta x\right) \cdot L+\ldots+\left(a_{20}-b_{20} \Delta x\right) \cdot H^{3} \\
& \rho_{3}^{c}(P, L, H)=\left(c_{1}-d_{1} \Delta y\right)+\left(c_{2}-d_{2} \Delta y\right) \cdot P+\left(c_{3}-d_{3} \Delta y\right) \cdot L+\ldots+\left(c_{20}-d_{20} \Delta y\right) \cdot H^{3}
\end{aligned}
$$

Effectively, all original terms in the numerator of each expression in Equation 3-20 are modified. In the equation of $\mathrm{x}$, each coefficient $a_{K}$ is replaced by $\left(a_{K}-b_{K} \Delta x\right)$. And the case for the y equation is similar. In this way, the vendor-supplied RPCs can be replaced using the method mentioned above. The bias inside the initial RPCs could be corrected. This would be much more convenient for some softcopy photogrammetric workstations, which only support the use of RPCs to do the 3D reconstruction of IKONOS imagery.

The bias compensation model defined in the ground domain is given as:

$$
\begin{aligned}
& P=a_{0}+a_{1} P_{R F}+a_{2} L_{R F}+a_{3} H_{R F} \\
& L=b_{0}+b_{1} P_{R F}+b_{2} L_{R F}+b_{3} H_{R F} \\
& H=c_{0}+c_{1} P_{R F}+c_{2} L_{R F}+c_{3} H_{R F}
\end{aligned}
$$

where

$a_{0}, a_{1}, a_{2}, a_{3}, b_{0}, b_{1}, b_{2}, b_{3}, c_{0}, c_{1}, c_{2}, c_{3}$ are the adjustable coefficients;

$P_{R F}, L_{R F}, H_{R F}$ are the ground coordinates calculated from the vendor supplied 
RPCs and

$P, L, H$ is the ground coordinates after bias compensation;

The bias compensation in the ground domain use the method to correct the final ground coordinates use the adjustable parameters calculated from the GCPs. In this method, the adjustable parameters have no relationship to the imaging geometry. After using the softcopy photogrammetry workstation to finish the DEM or orthophoto generation, there is still a lot of work to do with the bias compensation using this method.

Figure 3-5 shows the stereo reconstruction steps when using the RFM.

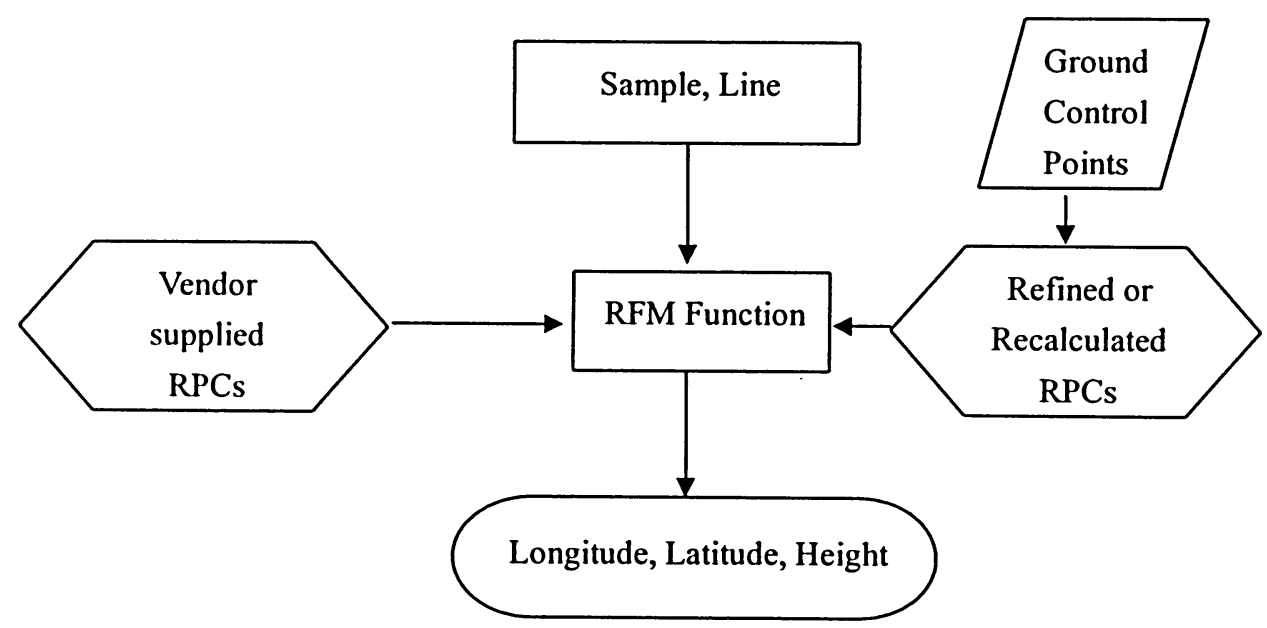

Figure 3-5 Processing steps of 3D reconstruction using the RFM model.

\subsubsection{D Physical Model}

The IKONOS model in the PCI Geomatica OrthoEngine was developed first for multi-sensor images at the CCRS and given by Toutin (1995). This method was then 
adopted to high resolution images (Toutin and Cheng, 2000; Toutin et al., 2001). As it is described in OrthoEngine manuals, the proprietary sensor model of this software utilizes the basic information available for the metadata and image files. For example, approximate sensor viewing angles can be computed using the nominal collection elevation and azimuth in addition to the nominal ground resolution. It further reflects the physical reality of the complete viewing geometry and corrects all the geometric distortions due to the platform, sensor and Earth that occur during the imaging process, and also any deformations of the cartographic projection.

The geometric modeling represents the well-known collinearity condition (and coplanarity condition for stereo model), and takes into account the different distortions relative to the global geometry of viewing (Toutin, 2003), i.e.,

- The distortions relative to the platform (position, velocity, orientation),

- The distortions relative to the sensor (orientation angles, instantaneous field of view, detection signal integration time),

- The distortions relative to the Earth (geoid-ellipsoid, including elevation), and

- The deformations relative to the cartographic projection (ellipsoid-cartographic plane).

As stated by Toutin, for the 3D physical model, the collinearity equations for a ground point are first written in the instrumental reference system and converted into the cartographic projection system using elementary transformations (Toutin, 1983). The 
following transformations are included in the 3D physical model (Toutin, 2003):

- Rotation from the sensor reference to the platform reference,

- Translation to the Earth's centre,

- Rotation which takes into account the platform time variation,

- Rotation to align the z-axis with the image centre $\left(\mathrm{M}_{0}\right)$ on the ellipsoid,

- Translation to the image centre,

- Rotation to align the y-axis in the meridian plane,

- Rotation to have $\mathrm{xM}_{0} \mathrm{y}$ tangent to the ellipsoid,

- Rotation to align the $\mathrm{x}$-axis in the image scan direction, and

- Rotation-translation into the cartographic projection.

The equation of this $3 \mathrm{D}$ physical model which gives the relationship between the $3 \mathrm{D}$ cartographic coordinates and the image coordinates provided by Toutin (2003) is described in Equation (3-24): 


$$
\begin{aligned}
& P p+y(1+\delta \gamma X)-\tau H-H_{0} \Delta T^{*}=0 \\
& X+\theta H / \cos \chi+\alpha q(Q+\theta X-H / \cos \chi)-Q \Delta R=0
\end{aligned}
$$

where

$$
X=(x-a y)\left(1+h / N_{0}\right)+b y^{2}+c x y
$$

and

$$
H=h-X^{2} / 2 N_{0}
$$

Each parameter is given using a mathematical formula (Toutin, 1983) that represents the physical realities of the full viewing geometry (satellite, sensor, Earth, map projection): where

$H$ is the altitude of the point corrected for Earth's curvature,

$H_{0}$ is the satellite elevation at the image center line,

$N_{0}$ is the normal to the ellipsoid,

$a$ is mainly a function of the rotation of the Earth,

$\alpha \quad$ is the instantaneous field of view,

$p, q$ are the image coordinates,

$P, Q$ are the scale factors in $y$ and $x$, respectively,

$\tau$ and $\theta$ are a function of the leveling angels in $y$ and $x$, respectively,

$\Delta T^{*}$ and $\Delta R$ are the non-linear variations in attitude if they exist,

$x, y$ and $h$ are the ground coordinates and,

$b, c, \chi$ and $\delta \gamma$ are second-order parameters, which are a function of the total 
geometry.

Toutin (2003) also demonstrated that each of these parameters is in fact the combination of several correlated variables of the viewing geometry, so that the number of unknown parameters has been reduced to an independent decorrelated set:

- The orientation of the image is a combination of the platform heading due to orbital inclination, the yaw of the platform, and the convergence of the meridian;

- The scale factor in the in-track direction is a combination of the velocity, the altitude and the pitch of the platform, the detection signal time of the sensor, and the component of the Earth rotation in the in-track direction; and

- The leveling angle in the across-track direction is a combination of platform roll, the viewing angle, the orientation of the sensor, Earth curvature, etc.

As reported by Toutin (2003), this method has been successfully applied to visible and infrared data sets with three to six GCPs needed. And it is also reported to robust and not sensitive to GCP distribution.

\subsubsection{Accuracy Assessment of the Sensor Model}

The accuracy of each sensor models mentioned above would be evaluated by the residuals in the GCPs and the errors in the ICPs. The assessment relies on two aspects, 
one is the calculation of the RMSE and the other is the standard deviation (STDE) of the calculated errors to describe the distribution of the errors in the ICPs.

RMSE is a measure of how closely a data set matches the actual world. The definition of RMSE is provided below:

$R M S E=\sqrt{\frac{\sum_{i=1}^{n} E_{i}{ }^{2}}{n}}$

where

$E_{i}$ is error at check point $i$

$n$ is the number of the check points

The STDE is the measure that is most often used to describe variability in data distributions. It can be thought of as a rough measure of the average amount by which observations deviate on either side of the mean. It is directly related to the variance and is simply its square root. Unlike the variance, the STDE describes variability in the original units of measurement. This is because the squaring function and square root function cancel each other. The final answer, however, remains positive because the initial squaring function eliminated the negative deviations. The STDE is given as: 
$S T D E=\sqrt{\frac{\sum_{i=1}^{n}\left(E_{i}-\bar{E}\right)^{2}}{n}}$

where

$E_{i}$ is the error at check point $i$

$\bar{E}$ is the mean error of all the check points, and

$\mathrm{n}$ is the number of points.

\subsection{Automatic Image Matching}

In the selection and calculation of the stereo image pair, math model is important since it determines the accuracy of the $3 \mathrm{D}$ ground coordinates and, thus, affects the accuracy of the final DEM. From the calculation steps described in Section 3.2, it can be clearly seen that the conjugate image points must be found first to calculate the ground coordinates of that point. The automatic image matching technique is used to assist the searching for the conjugate points on the stereo image pair.

Stereo image matching, in particular, is of fundamental importance to photogrammetry. Any automated system for $3 \mathrm{D}$ point positioning must include a potent procedure for stereo image or dual image matching. One popular way for the conjugate search is through epipolar resampling. Epipolar relationship can be established between stereo 
images and this is very useful in stereo matching. Epipolar relationship or epipolar geometry refers to the specific properties of stereo images taken by perspective cameras and can be explained in Figure 3-6. An epipolar plane can be defined by a point $q$ in the left image, the left centre of perspective $S$ and the right center of perspective $S^{\prime}$. The point q is projected to points on a straight line lq' ('epipolar' line) in the right image and the point q', the conjugate point of q, will lie on the epipolar line lq. Furthermore, all the points on one epipolar line $\mathrm{lq}$ in the left image are mapped uniquely onto the corresponding epipolar line lq' in the right image and vice versa.

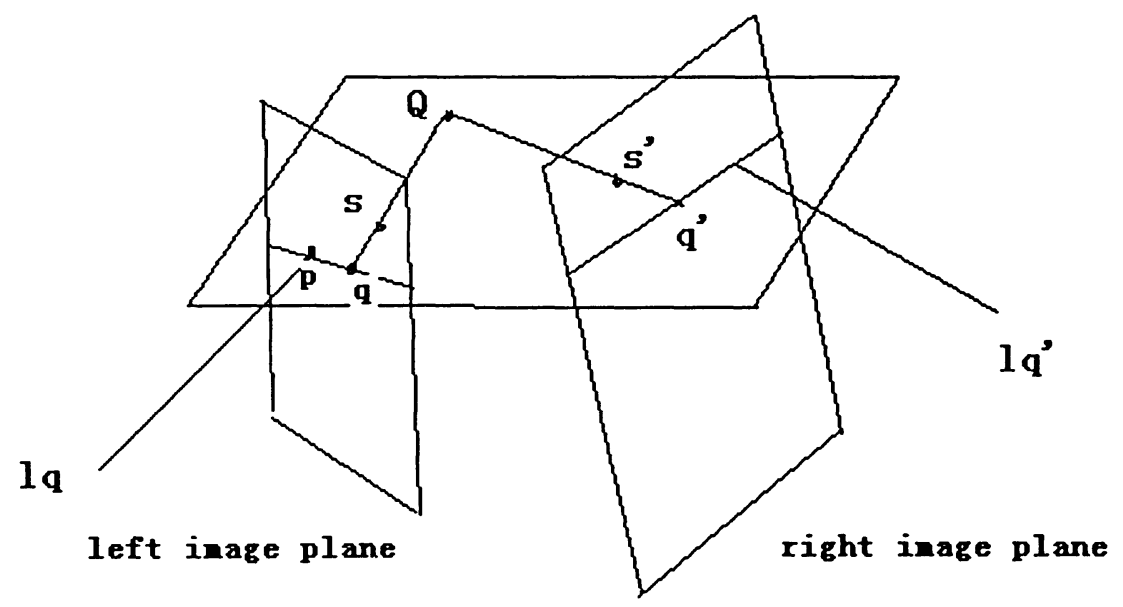

Figure 3-6 Epipolar geometry.

Using these properties, the original images can be transformed so that the epipolar lines coincide with (typically) image row direction. Then, the conjugate search can be confined only in the row direction. Through epipolar resampling, the problem of $2 \mathrm{D}$ conjugate search can be reduced to 1D. In fact, this method is one of the most popular techniques for stereo matching perspective images (Pollard, et al., 1985). However, 
according to the findings on the linear pushbroom image geometry, it is not theoretically possible for linear pushbroom images to be perfectly epipolar resampled (Kim, 2000). This rather disappointing fact is originated from the uniqueness of the linear pushbroom image geometry, in which, one point in the left image is not projected to the right image by straight lines but hyperbola-like curves (Gupta and Hartley, 1997). Moreover, all points on one epipolar curve in the left image do not mapped to identical epipolar curve in the right image.

As mentioned earlier, true epipolar resampling for linear pushbroom images is not feasible. If the linear pushbroom images are regarded as perspective images and perform epipolar resampling, the resampled images will possess not only $\mathrm{x}$ - but also $y$-parallex. However, if the scale of resampled images is reduced until y-parallex becomes negligible, it can be assumed that the reduced images are same as true epipolar images (Cheng and Toutin, 1997). At the reduced scale, a 1D conjugate search method can be applied. At higher scales, the match results at reduced scales can be used to get refined match results. At each scale, the match result by $1 \mathrm{D}$ search can be refined using least-squares correlation. This 'pseudo epipolar resampling coupled with a pyramidal approach and/or 2D search by least-squares correlation may potentially overcome the errors induced by epipolar resampling linear pushbroom images compulsively.

The PCI Geomatica OrthoEngine software provides the capability of automatic image matching technique, used to extract the elevation parallax. Once the GCPs are collected, 
and the geometric model is computed for each image of the stereo pair, the next step is to generate quasi-epipolar curve images from the stereo pair. An area based automated image matching procedure is then used to extract the elevation parallax, and produce the DEM through a comparison of the respective gray values on each of these images. This procedure utilizes a mean normalized cross-correlation matching method with a multi-scale strategy to match the image using the statistics collected in defined windows. Matching is performed by considering the neighborhood surrounding a given pixel in the left quasi-epipolar image (thus forming a template) and moving this template within a search area on the right epipolar image until a position is reached which gives the best match. The actual matching method employed with PCI Geomatica OrthEngine software generates correlation coefficients between 0 and 1 for each match pixel, where 0 represents a total mismatch and 1 represents a perfect match. A $2^{\text {nd }}$-order surface is then fitted to the maximum correlation coefficients to find the match position to sub-pixel accuracy. The difference in location between the center of the template and the best matched pixel position gives the disparity or parallax arising from the terrain relief, from which the absolute elevation value is then computed. The advantage of using this procedure is that the search for the matching pixels is effectively limited to the epipolar line, thus significantly improving the algorithm's efficiency and accuracy. A further advantage arising from the matching method used is that it tolerates any spatially invariant, linear radiometric relationship between the two images. 


\subsection{DEMs Generation and Editing}

The basic principle for DEM generation is based on image matching. Following the matching process, conjugate points undergo an intersection procedure to derive the ground coordinates of the corresponding object points. The RFM or the $3 \mathrm{D}$ physical model now could be used for such computation. The ground coordinates can be derived through a least-squares adjustment procedure. So far, the ground coordinates of the matched interest points, which passed the consistency check, are derived through space intersection. These points are irregularly distributed and are not dense enough to represent the object space. Therefore, they need to be interpolated.

The output elevations are not computed for the pixels where the image matching fails to find the corresponding pixel in the reference image, resulting into some failure areas. In the case of small and scattered failures the software uses interpolation and computes the most probable values for these points.

DEMs may contain pixels with failed or incorrect values. This is why the DEM editing is needed to smooth out the irregularities and create a more visually pleasing DEM. The purpose of this procedure is to remove blunders, to fill in the mismatched area, and to smooth the DEM. Editing DEM requires an understanding of the desired results combined with insightful artistry to achieve the desired results. Every DEM presents a variety of problematic situations. 


\section{RESULTS AND DISCUSSION}

The first step towards automatic extraction of DEMs in this study is to collect GCPs. The description of the GCP selection and measurements are included in Section 4.1. The second step is the 3D reconstruction using the RFM the 3D physical model, respectively. The results and the accuracy discussion of the 3D reconstruction using different sensor models are given in Section 4.2. Based on the 3D reconstruction results from Section 4.2, the third step, automatic image matching and DEMs generation, are provide in Section 4.3. In Section 4.4, the result of the forth step, DEMs editing, is presented. And Section 4.5 gives a summary of this chapter.

\subsection{GCP Collection}

A total of $63 \mathrm{GCPs}$ and their corresponding image coordinates on both the left and the right image of the IKONOS stereo pair were measured in this study. Some of these points were selected at landmarks and the others were at intersections between sidewalks and roads. All of the GCPs were located on the surface of the terrain. Figure 4-1 shows an example of how a GCP was determined and chosen. The measurement error of the image coordinates of GCPs is less than 0.5 pixel. Figure $4-2$ illustrates the distribution of the GCPs measured on the central subsection of the IKONOS stereo images. Table 4-1 lists these GCPs with their 3D ground image coordinates. The GCPs are in the UTM projection, Zone 17, WGS84 ellipsoid, which is as same as the 
IKONOS stereo images. The elevations are referenced to the ellipsoid.

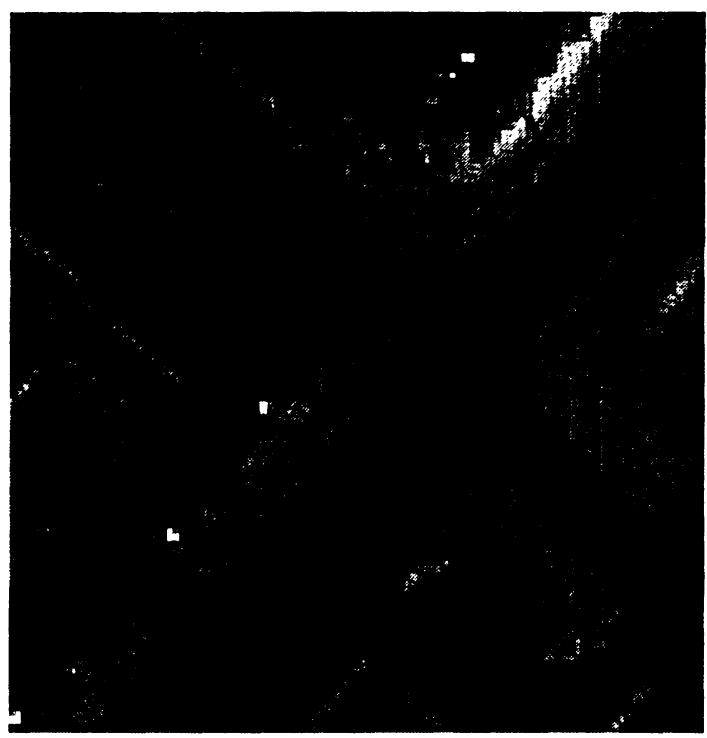

Figure 4-1 An example of the selection of one GCP.

Table 4-1 GCPs with their 3D ground coordinates and 2D image coordinates

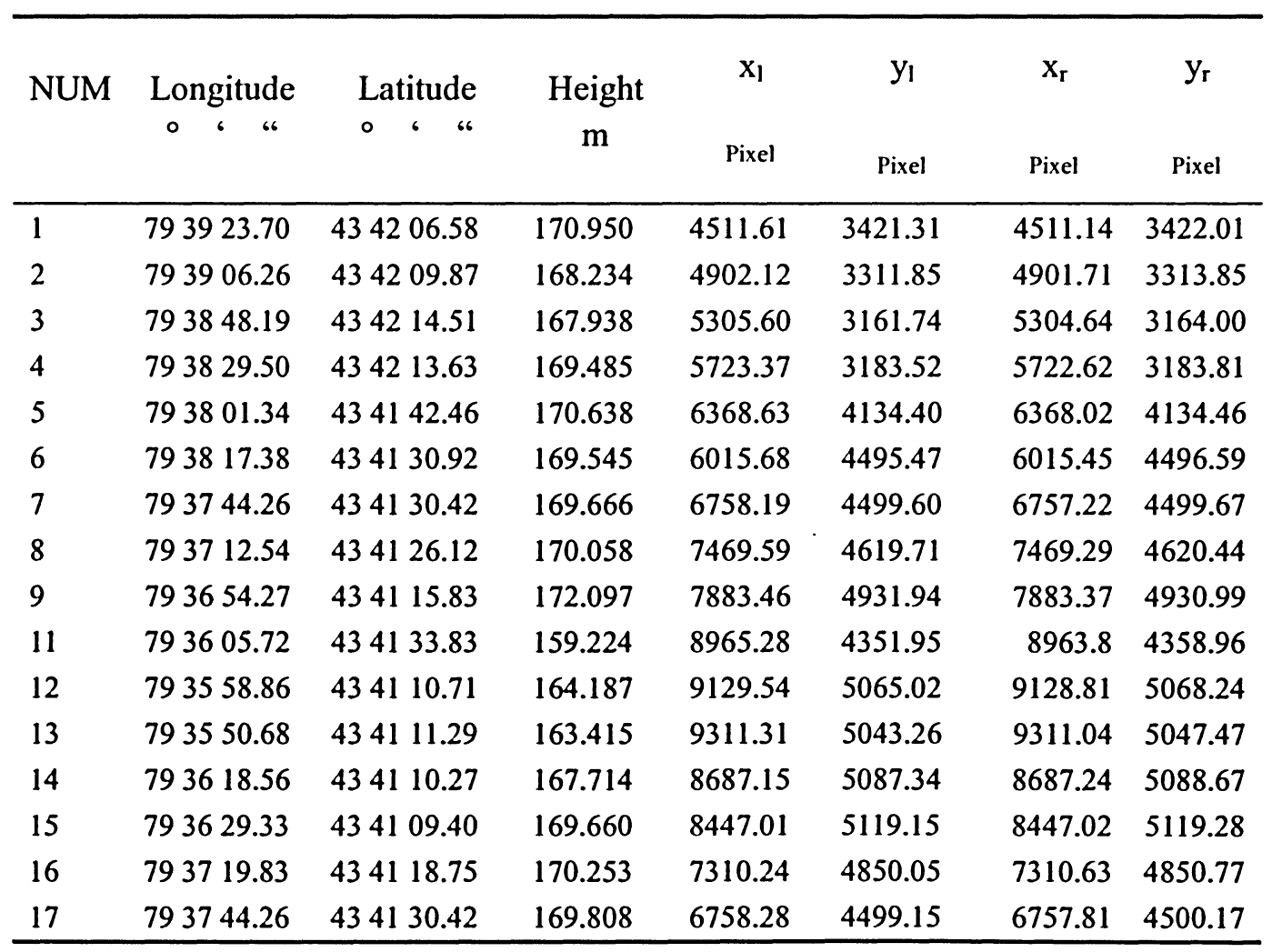




\begin{tabular}{|c|c|c|c|c|c|c|c|}
\hline 18 & 793753.00 & 434136.95 & 171.076 & 6557.97 & 4301.06 & 6557.44 & 4300.83 \\
\hline 19 & 793834.52 & 434133.69 & 169.438 & 5630.66 & 4416.16 & 5630.58 & 4417.58 \\
\hline 20 & 793823.45 & 434123.06 & 169.177 & 5883.67 & 4741.62 & 5883.99 & 4742.75 \\
\hline 21 & 793933.62 & 434131.75 & 170.067 & 4307.94 & 4498.59 & 4307.81 & 4499.02 \\
\hline 22 & 793941.84 & 434137.88 & 171.705 & 4119.98 & 4313.18 & 4119.64 & 4313.32 \\
\hline 23 & 793934.61 & 434144.38 & 172.289 & 4278.20 & 4111.04 & 4277.91 & 4109.55 \\
\hline 24 & 793957.79 & 434135.54 & 172.916 & 3763.24 & 4391.96 & 3764.31 & 4389.92 \\
\hline 25 & 794009.68 & 434111.62 & 175.209 & 3510.10 & 5134.41 & 3510.64 & 5132.01 \\
\hline 26 & 794002.52 & 434102.19 & 174.589 & 3673.26 & 5423.00 & 3673.76 & 5421.34 \\
\hline 27 & 794014.38 & 434052.43 & 175.109 & 3413.00 & 5727.97 & 3413.58 & 5725.94 \\
\hline 28 & 794008.21 & 434047.51 & 174.596 & 3555.13 & 5878.25 & 3555.45 & 5876.07 \\
\hline 29 & 793948.03 & 434027.78 & 171.153 & 4016.30 & 6477.68 & 4016.41 & 6477.79 \\
\hline 30 & 793836.56 & 434029.77 & 159.396 & 5620.24 & 6387.05 & 5618.8 & 6393.28 \\
\hline 31 & 793754.40 & 434010.68 & 166.314 & 6571.03 & 6961.56 & 6570.87 & 6963.81 \\
\hline 32 & 793805.33 & 434019.22 & 166.703 & 6322.68 & 6702.38 & 6322.48 & 6704.82 \\
\hline 33 & 793818.71 & 434030.68 & 165.023 & 6017.45 & 6353.32 & 6017.13 & 6356.31 \\
\hline 34 & 793808.81 & 434036.00 & 169.726 & 6235.92 & 6186.32 & 6235.82 & 6187.66 \\
\hline 35 & 793816.90 & 434132.66 & 169.560 & 6026.49 & 4442.66 & 6025.57 & 4443.03 \\
\hline 36 & 793824.83 & 434124.11 & 169.281 & 5852.57 & 4709.00 & 5852.46 & 4710.03 \\
\hline 37 & 793807.74 & 434112.55 & 168.089 & 6240.94 & 5058.25 & 6240.11 & 5059.81 \\
\hline 38 & 793757.67 & 434200.20 & 171.623 & & & & \\
\hline 39 & 793757.07 & 434148.40 & 170.671 & 6460.99 & 3949.23 & 6461.1 & 3949.24 \\
\hline 40 & 793742.37 & 434136.64 & 171.519 & 6796.72 & 4306.94 & 6796.33 & 4306.76 \\
\hline 41 & 793724.36 & 434123.83 & 171.019 & 7205.87 & 4695.87 & 7206.49 & 4695.68 \\
\hline 42 & 793522.07 & 433958.43 & 159.543 & 9992.14 & 7279.17 & 9990.38 & 7285.5 \\
\hline 43 & 793539.30 & 433954.72 & 160.957 & 9607.89 & 7401.27 & 9605.67 & 7406.58 \\
\hline 44 & 793555.68 & 433945.92 & 157.784 & 9247.09 & 7678.12 & 9244.67 & 7685.71 \\
\hline 45 & 793627.72 & 433939.79 & 160.367 & 8532.05 & 7879.72 & 8530.1 & 7885.58 \\
\hline 46 & 793641.33 & 433925.69 & & 8234.80 & 8319.39 & 8232.88 & 8326.05 \\
\hline 47 & & & & & & 7781.6 & \\
\hline 49 & 793813.13 & 433842.87 & 159.822 & 6198.27 & 9673.54 & 6197.78 & 9680.34 \\
\hline 50 & 793844.02 & 433912.29 & 164.963 & 5490.57 & 8780.41 & 5489.22 & 8783.32 \\
\hline 51 & 793822.19 & 433946.48 & 161.538 & 5962.47 & 7716.23 & 5961.7 & 7722.22 \\
\hline 52 & 793725.93 & 433940.26 & 160.879 & 7226.61 & 7887.51 & 7225.48 & 7892.82 \\
\hline 53 & 793710.68 & 433950.24 & 162.011 & 7563.43 & 7573.74 & 7562.04 & 7578.68 \\
\hline 54 & 793654.13 & 434015.57 & 166.031 & 7918.77 & 6788.42 & 7918.71 & 6791.22 \\
\hline 55 & 793552.21 & 434044.79 & 165.910 & 9289.55 & 5862.33 & 9289.42 & 5865.47 \\
\hline 56 & 793606.66 & 434034.24 & 165.410 & 8971.14 & 6193.00 & 8971.33 & 6195.72 \\
\hline 57 & 793526.59 & 434033.72 & 160.878 & 9870.64 & 6191.16 & 9868.87 & 6196.52 \\
\hline 58 & 793838.28 & 433955.49 & 165.836 & 5596.52 & 7446.50 & 5595.56 & 7448.69 \\
\hline 59 & 793804.42 & 434019.87 & 166.725 & 6342.43 & 6682.30 & 6342.47 & 6683.94 \\
\hline 60 & 793721.18 & 434035.51 & 172.033 & 7300.88 & 6186.37 & 7301.53 & 6185.38 \\
\hline 61 & 793806.39 & 434057.19 & 169.058 & 6278.92 & 5531.88 & 6278.73 & 5532.23 \\
\hline 62 & 794001.39 & 433959.58 & 170.543 & 3731.74 & 7351.56 & 3731.56 & 7352.24 \\
\hline
\end{tabular}




\begin{tabular}{llllllll}
\hline 63 & 793934.36 & 433945.25 & 173.55 & 4343.61 & 7786.10 & 4343.68 & 7784.35 \\
64 & 793905.97 & 434001.03 & 169.955 & 4971.82 & 7288.15 & 4972.93 & 7288.08 \\
65 & 793649.53 & 434056.33 & 183.663 & 7996.32 & 5536.25 & 7997.88 & 5529.07 \\
\hline
\end{tabular}

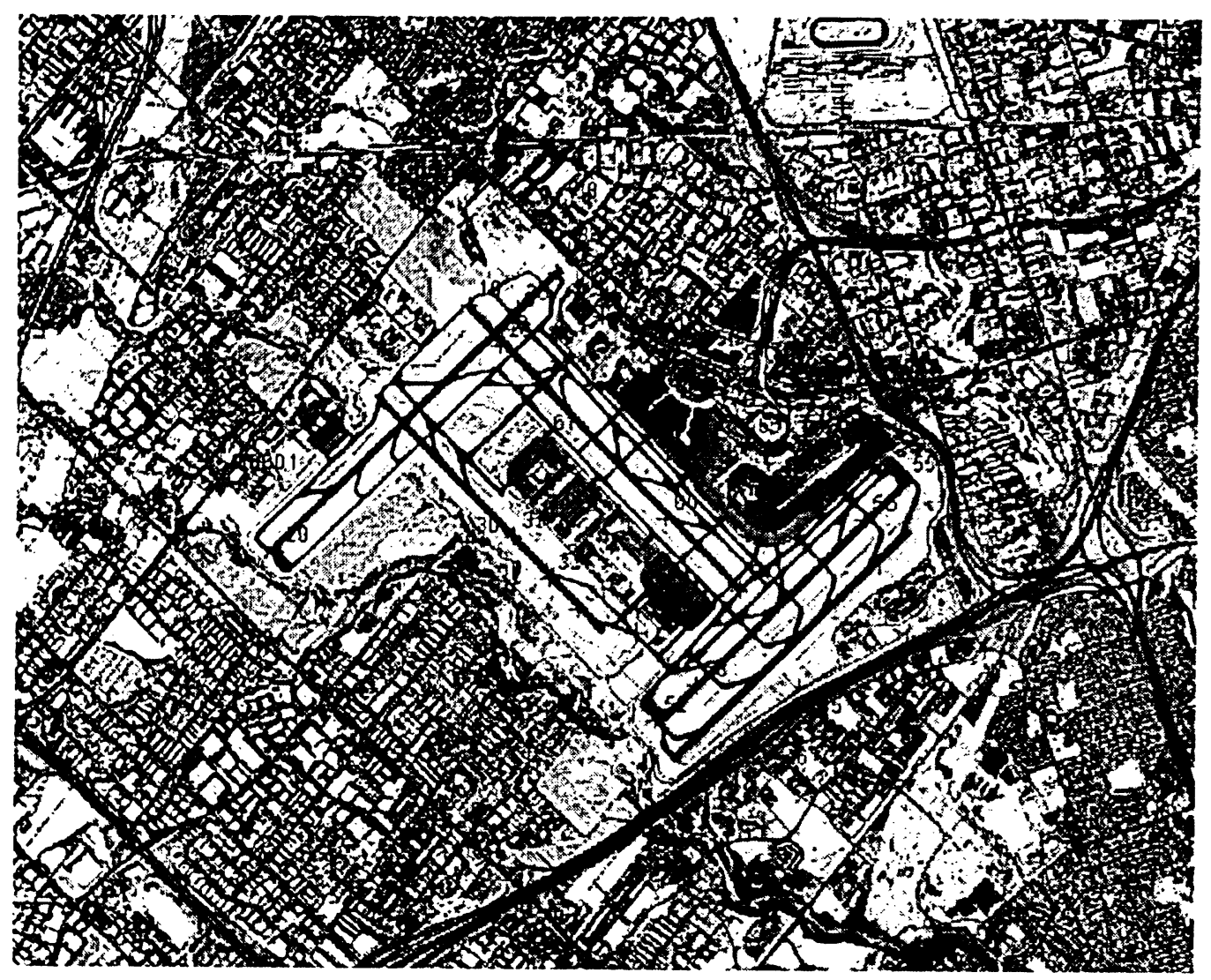

Figure 4-2 GCPs distributed on the central subsection of the left image of the

IKONIOS stereo pair.

\subsection{D Reconstruction}

$3 \mathrm{D}$ reconstruction using different sensor models is one of the most important steps in automatic extraction of DEMs from IKONOS stereo images. The selection and accuracy of the sensor model will directly affect the accuracy of the final DEMs derived. Therefore, many researchers focus their efforts on the sensor models. In this section, the results from the calculation of the RFM and the $3 \mathrm{D}$ physical model are presented and 
discussed. The methods used to evaluate sensor models are mainly based on the RMSE of the GCPs and ICPs from the stereo bundle adjustment results. The residuals in the GCPs reflect the input error of the stereo bundle adjustment. However, unbiased validation of the positioning accuracy has to be realized with ICPs, which were not used in the 3D parametric model calculation. Different GCPs and ICPs configurations were used to evaluate the sensor models and to find the relationship between the $3 \mathrm{D}$ position accuracy and the number and distribution of the GCPs.

\subsubsection{D Reconstruction Using RFM}

As mentioned in Section 3.4.1, 3D reconstruction could be achieved using the vendor-supplied RPCs. To assess the accuracy and error distribution of the results using the original RPCs, all the 63 ground points were used as checkpoints. The RMSE on the 63 checkpoints and the STDE of the errors were calculated and listed in Table 4-2. Figure 4-3 shows the planimetric and elevation error vectors at each checkpoint, respectively.

Table 4-2 RMSE and STDE on the ICPs without GCP

\begin{tabular}{ccccc}
\hline 63 ICPs & RMSE $(\mathrm{m})$ & STDE $(\mathrm{m})$ & Max Error $(\mathrm{m})$ & Min Error $(\mathbf{m})$ \\
\hline $\mathbf{X}$ & 2.1 & 0.68 & 3.7 & 0.3 \\
$\mathrm{Y}$ & 4.9 & 0.70 & 6.3 & 2.7 \\
$\mathrm{Z}$ & 12.3 & 0.75 & 13.6 & 10.3 \\
\hline
\end{tabular}




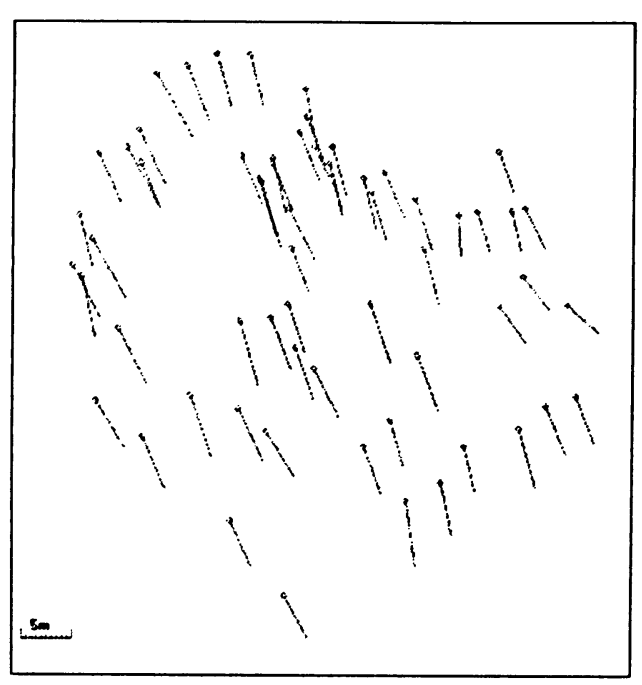

(a)

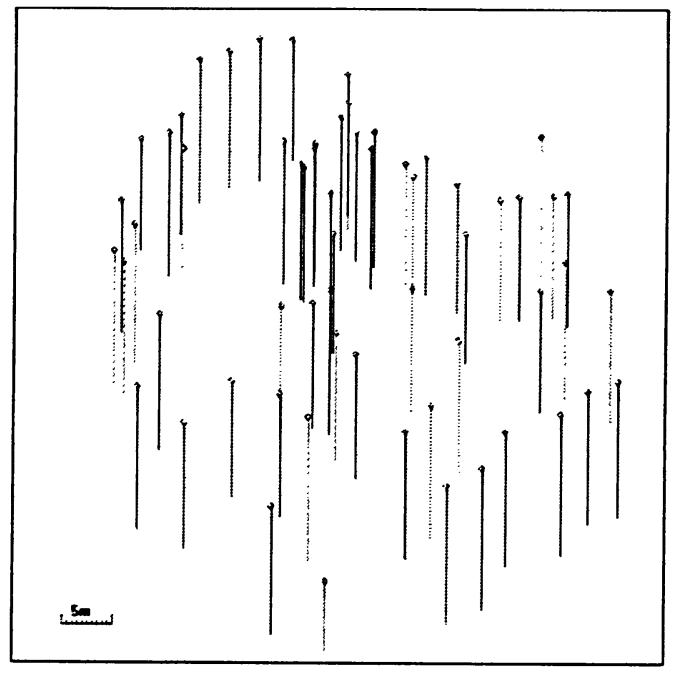

(b)

Figure 4-3 Error vectors of (a) planimetry and (b) elevation at ICPs, calculated using vendor-supplied RPCs.

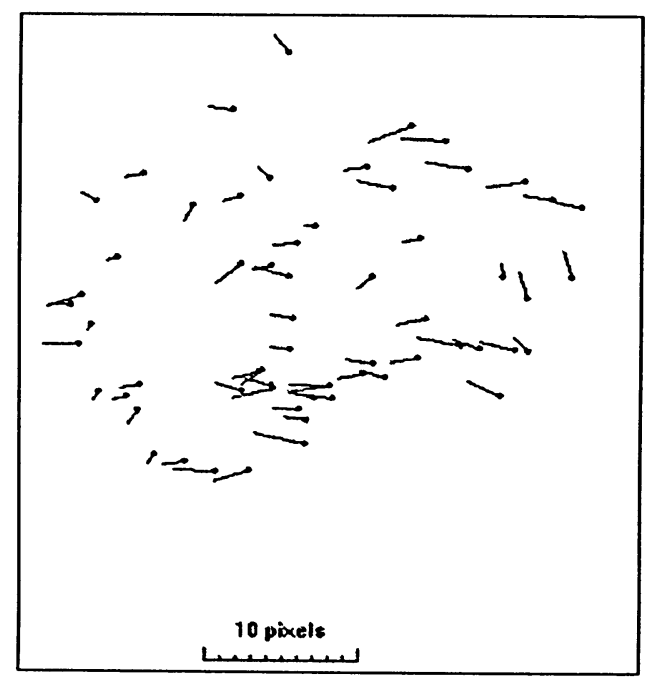

(a)

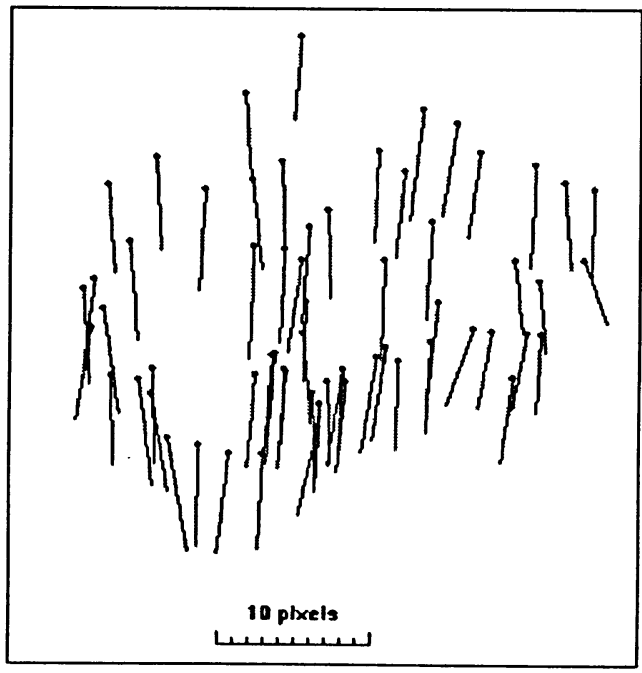

(b)

Figure 4-4 Bias vectors of image coordinates of (a) left and (b) right image, calculated using vendor-supplied RPCs.

The RMSE values without GCP were approximately $2 \mathrm{~m}$ in X, $4 \mathrm{~m}$ in $\mathrm{Y}$, and $12 \mathrm{~m}$ in Z 
direction, respectively, which present 3D reconstruction accuracy of the IKONOS in-track stereo images without the use of GCPs. As shown in Figure 4-3 and Table 4-2, although the RMSE is significant in $\mathrm{X}, \mathrm{Y}$ and $\mathrm{Z}$ directions, the STDE values are much smaller than the RMSE values. This indicates that a high degree of invariance of the ground point biases is inherent in RPCs derived results without the aid of GCPs. These biases could also be seen in the image domain as showed in Figure 4-4.

To improve the geometric accuracy of 3D reconstruction and to find the characteristic of the bias and its relationship to the number and distribution of GCPs, a set of tests were conducted and the results are discussed below. Two methods were selected for this purpose: (1) to use the vendor-supplied RPCs as the initial values to recalculate the RPCs with ground control information, and (2) to refine the vendor-supplied RPCs by compensating for the bias as calculated from a few number of GCPs or by removing the bias after the 3D reconstruction.

\section{(1) Recalculation of RPCs}

There is an apparent bias in the result of 3D reconstruction performed by the vender-supplied RPCs. To improve the accuracy, one method that could be used involves the recalculation of the RPCs used in the 3D reconstruction. A large number of GCPs are required to recalculate the 78 coefficients (20 in the numerator and 19 in the denominator for $x$ and $y$, respectively) in the rational function of each image of the IKONOS stereo pair. By using the method mentioned in Section 3.4.1, each stereo GCP 
will provide four error equations as defined in Equation 3-21. That means at least 39 GCPs are required to calculate the total 78 RFCs in both of the stereo images. In this study, 45 points were used as GCPs to calculate the new coefficients of each image. The remaining 18 points were used as ICPs. The RMSE of the GCPs and ICPs are listed in Table 4-3. Figure 4-5 shows the distribution of the GCPs and ICPs and the errors associated with them.

Table 4-3 RMSE at the GCPs and ICPs using the new calculated RPCs

\begin{tabular}{cccc}
\hline & \multicolumn{3}{c}{ RMSE of Ground Points (m) } \\
\hline Point Type & $\mathrm{X}$ & $\mathrm{Y}$ & $\mathrm{Z}$ \\
GCPs & 0.3 & 0.4 & 0.6 \\
ICPs & 0.7 & 0.4 & 0.8 \\
\hline
\end{tabular}

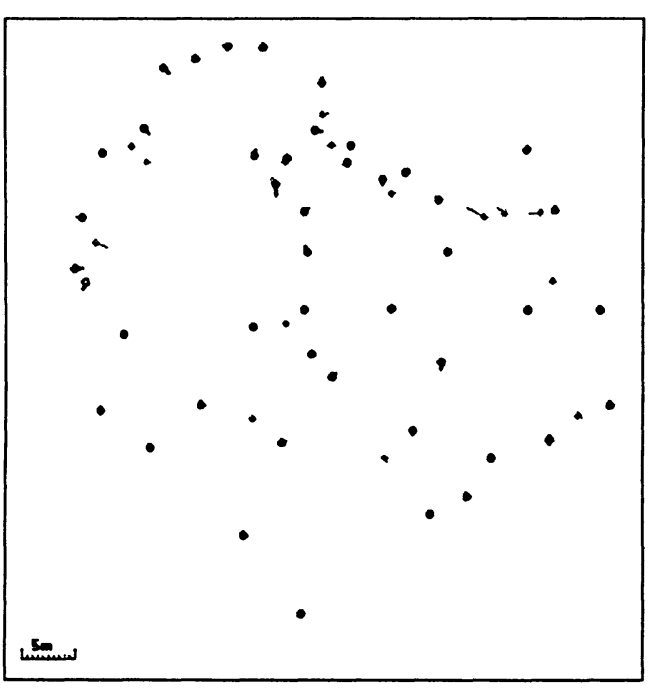

(a)

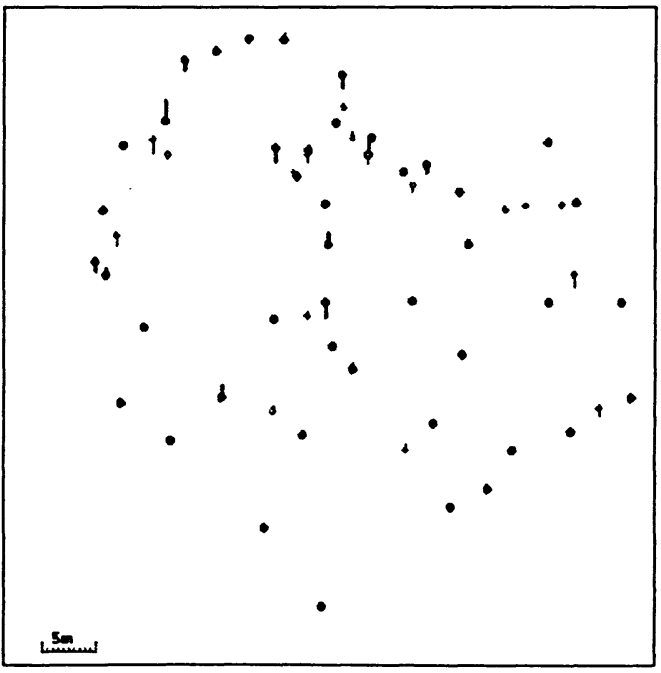

(b)

Figure 4-5 Error vectors in (a) planimetry and (b) elevation of GCPs (marked in black) and ICPs (marked in gray) using calculated RPCs. 
The results obtained after using recalculated RPCs look good and the RMSE on ICPs is less than $1 \mathrm{~m}$. Also, the calculation is stable when using the vendor-supplied RPCs as the initial values to recalculate the RPCs in the numerator and denominator separately. The newly calculated coefficients are at the same order of magnitude as the original ones. The above results were obtained after two iterations. The disadvantage of this method is that a large number of GCPs is needed, which may not be available all the time. To this end, another method called bias compensation was used, which could reduce the number of the GCPs required while achieving the same accuracy level of $3 D$ reconstruction.

\section{(2) Bias Compensation in RFM}

As mentioned in Section 3.4.1, the bias compensation could be achieved both in the image domain and ground domain. Several tests were done to determine how many GCPs are needed to remove the bias. And the advantage and disadvantage of the approach to bias compensation in image domain and ground domain are also discussed.

In the test of bias compensation in image domain, the selection of one GCP in the centre, three GCPs at the edge and four GCPs at the comers of the point distribution of the ground points are used to test the effect of the quantity and distribution of GCPs on the $3 D$ reconstruction results. Figure 4-6 shows the case of bias compensation using only one GCP in the centre. The error vectors of the checkpoints are plotted in gray and the selected GCP is marked in black. 


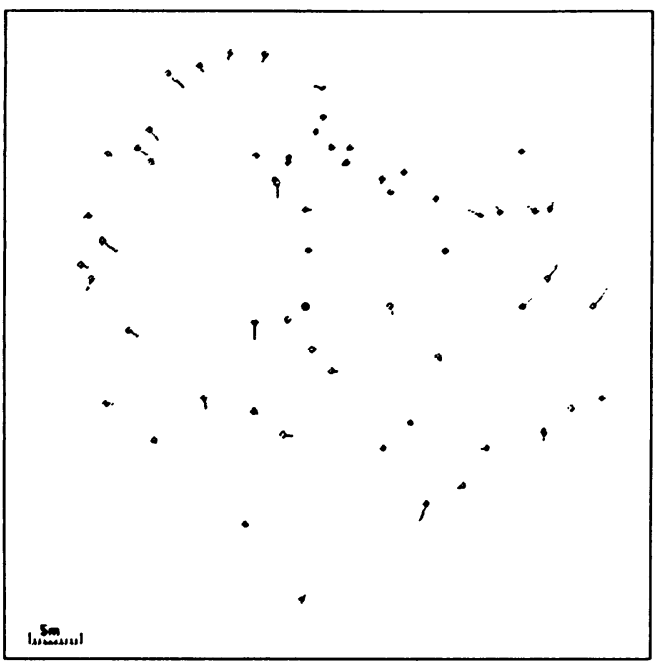

(a)

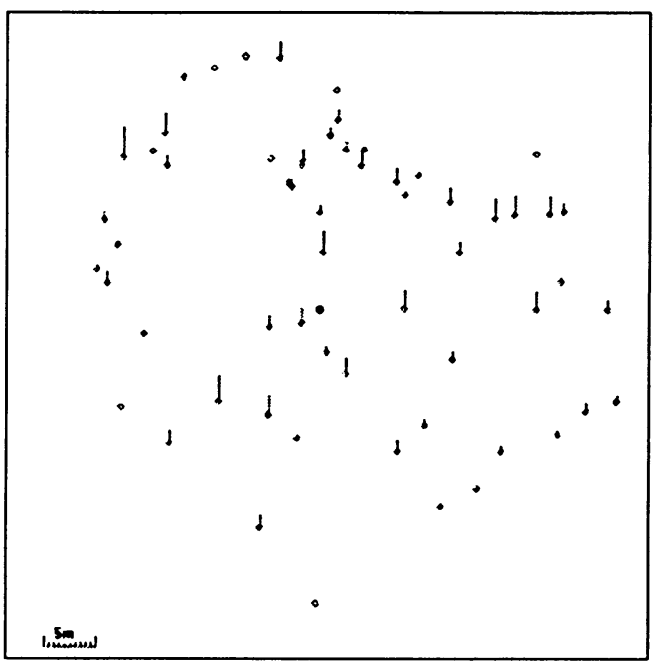

(b)

Figure 4-6 Error vectors in (a) planimetry and (b) elevation of ICPs using 1 GCP in the centre to remove the bias in image domain.

From the plot of the error vectors, it could be found that with only one GCP in the centre, both the planimetry and elevation accuracy on the ICPs have been greatly improved. Compared to the original results without GCP, the bias has been compensated well. The direction of the error vector of each checkpoint does not point to the same direction as they were shown in Figure 4-3. As illustrated in Table 4-4, the RMSE on the ICPs in $\mathrm{X}$ and $\mathrm{Y}$ direction has been improved to submetre level and around $1 \mathrm{~m} \mathrm{in} \mathrm{Z}$ direction, which showed larger bias in the previous experience.

In the case of one GCP was used to calculate the bias compensation coefficients, only the coefficients $a_{0}$ and $b_{0}$ in the Equation 3-23 could be calculated since one GCP can form two equations and, thus, calculate two coefficients. It can also be said that the coefficients $a_{S}, a_{L}, b_{S}, b_{L}$ are not considered in the above performance. Although the 
result seems quite good with one GCP, further tests are still needed to find the significance of the coefficients $a_{S}, a_{L}, b_{S}, b_{L}$. And also with only one GCP, the user may take the risk that this used GCP may contain significant errors.

The following two tests were then performed to find whether the result will be improved more if more GCPs are used and the effect of the coefficients $a_{S}, a_{L}, b_{S}, b_{L}$ is also considered.

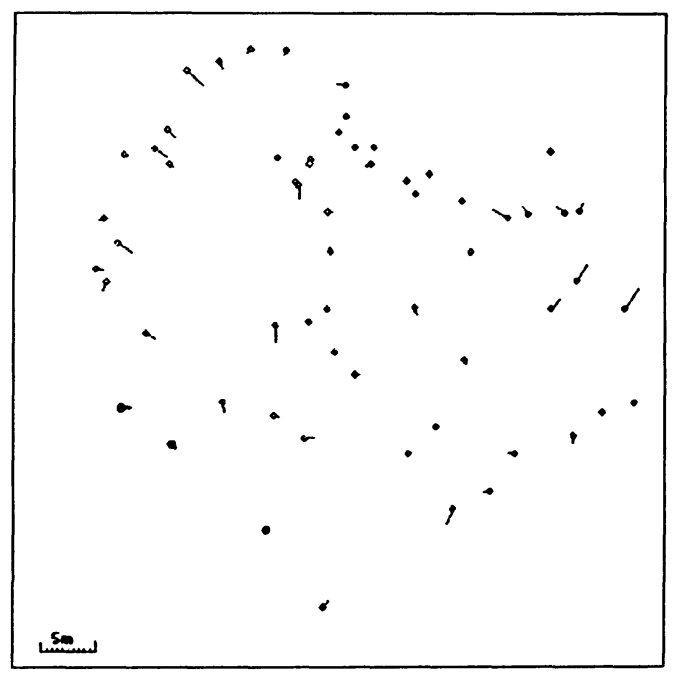

(a)

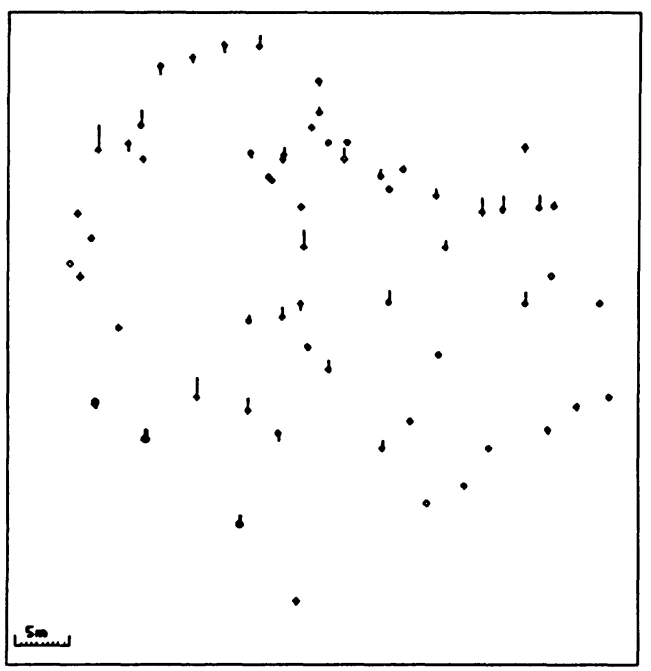

(b)

Figure 4-7 Error vectors in (a) planimetry and (b) elevation of ICPs using 3 GCPs at the edge to remove the bias in image domain.

First, 3 GCPs were used to calculate all the six coefficients in bias compensation described by Equation 3-23. These 3 GCPs were selected at the edge of the entire ground control distributed area. From the results shown in Figure 4-7 and the RMSE values on the checkpoints listed in Table 4-4, it is clear that the use of 3 GCPs could 
also achieve very good results in bias removing. The RMSE values are of the same order of magnitude as in the case of using one GCP. It also shows that the bad distribution of the GCPs does not affect the final RMSE values on the ICPs, which means that the coefficients $a_{S}, a_{L}, b_{S}, b_{L}$ contribute little to the bias calculation and the calculation of the biases is not sensitive to the distribution of the GCPs.

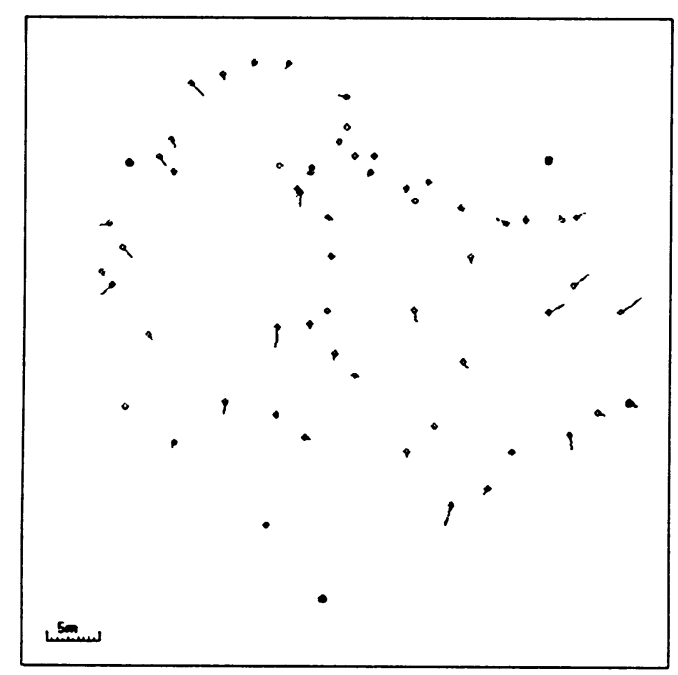

(a)

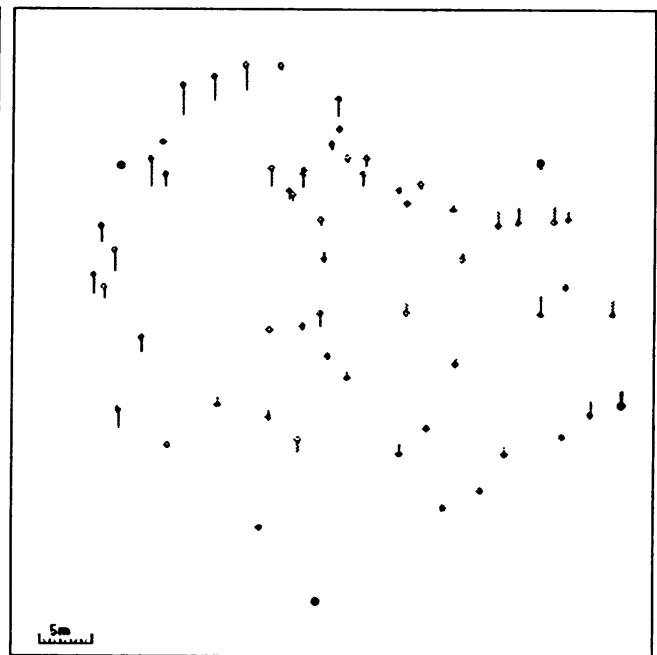

(b)

Figure 4-8 Error vectors in (a) planimetry and (b) elevation of ICPs using 4 GCPs at the corner to remove the bias in image domain.

To further test the effect of the GCP distribution, 4 GCPs with very good distribution (at the corner of the entire extent of the GCPs) were used to calculate the bias coefficients. By using these $4 \mathrm{GCPs}$, one redundant point is available, which will make the calculation more reliable.

From error vectors plot shown in Figure 4-8 and the RMSE values listed in Table 4-5, 
the result generated under the control of 4 GCPs is very similar to the other two tests mentioned above. The results demonstrate that, the distribution of GCPs leads to little effect, and contribute little on the coefficients $a_{S}, a_{L}, b_{S}, b_{L}$, which could be eliminated. Consequently, only a few GCPs are needed in the bias compensation in the image domain. This is very important to the end users who need good 3D reconstruction accuracy but have only small number of GCPs available.

Table 4-4 RMSE values of ICPs using different selection of GCPs to remove the bias in image domain

No. of

GCPs/ICPs

$1 / 62$

$3 / 60$

$4 / 59$
Model Used

RMSE of Checkpoints (m)

0.7

0.7

1.2

$$
\Delta y=b_{0}
$$

$\Delta x=a_{0}+a_{s} \cdot$ Sample $+a_{L} \cdot$ Line

$\Delta y=b_{0}+b_{s} \cdot$ Sample $+b_{L} \cdot$ Line

0.7

0.7

0.8

0.7

0.8

Finally, after the bias $\Delta x, \Delta y$ has been calculated by considering only the coefficients $a_{0}, b_{0}$ in Equation 3-24, the original RPCs could be refined using Equation 3-26. And these refined coefficients can be then used in the 3D reconstruction to obtain the 3D ground coordinates without bias, which are needed to generate a DEM in the later 
processing stage.

Further experiments of the bias calculation using the different numbers of GCPs were also performed and the results of the calculated biases $\Delta x, \Delta y$ in the stereo pair and the corresponding RMSE on the results are listed in Table 4-6. The biases in $x$ and $y$ directions using different numbers of GCPs are of the same order of magnitude respectively. However, the RMSE on the ICPs is not improved greatly if more GCPs are involved in the biases calculation. This has further proved the results achieved in the previous experiments.

Table 4-5 Computed image biases and RMSE of the ICPs

\begin{tabular}{|c|c|c|c|c|c|c|}
\hline \multirow{4}{*}{$\begin{array}{c}\text { No. of } \\
\text { GCPs/ICPs }\end{array}$} & \multirow{4}{*}{$\begin{array}{c}\text { Stereo } \\
\text { Pair }\end{array}$} & \multirow{3}{*}{\multicolumn{2}{|c|}{$\begin{array}{l}\text { Computed Image } \\
\text { Coordinates Biases } \\
\text { (pixels) }\end{array}$}} & \multicolumn{3}{|c|}{ RMSE of Checkpoints (m) } \\
\hline & & & & \multirow[b]{3}{*}{$X$} & \multirow[b]{3}{*}{$\mathrm{Y}$} & \multirow[b]{3}{*}{ Z } \\
\hline & & & & & & \\
\hline & & $\Delta x$ & $\Delta y$ & & & \\
\hline \multirow[t]{2}{*}{$2 / 61$} & Left & -1.9 & 0.4 & 0.7 & 0.8 & 0.8 \\
\hline & Right & -0.2 & -6.5 & & & \\
\hline \multirow[t]{2}{*}{$3 / 60$} & Left & -1.7 & 0.2 & 0.7 & 0.7 & 0.8 \\
\hline & Right & -0.0 & -6.6 & & & \\
\hline \multirow[t]{2}{*}{$4 / 59$} & Left & -1.6 & 0.3 & 0.7 & 0.7 & 0.8 \\
\hline & Right & 0.1 & -6.6 & & & \\
\hline \multirow[t]{2}{*}{$5 / 58$} & Left & -1.7 & 0.4 & 0.7 & 0.8 & 0.8 \\
\hline & Right & -0.1 & -6.5 & & & \\
\hline \multirow[t]{2}{*}{$7 / 56$} & Left & -1.4 & 0.4 & 0.7 & 0.8 & 0.7 \\
\hline & Right & -0.1 & -6.4 & & & \\
\hline \multirow[t]{2}{*}{$10 / 53$} & Left & -1.4 & 0.2 & 0.7 & 0.7 & 0.7 \\
\hline & Right & 0.0 & -6.7 & & & \\
\hline
\end{tabular}


Finally, a set of refined RPCs were calculated using 3 GCPs (No. 3, 20 and 42). The difference between the numerator of the refined and original RPCs are shown in Figures 4-9 - 4-12. From these four figures, it is clear that the biggest correction of the RPCs was done to the first coefficient in the numerator, which is the constant part in the RFM. As such, the error on this coefficient is the main source of these biases.

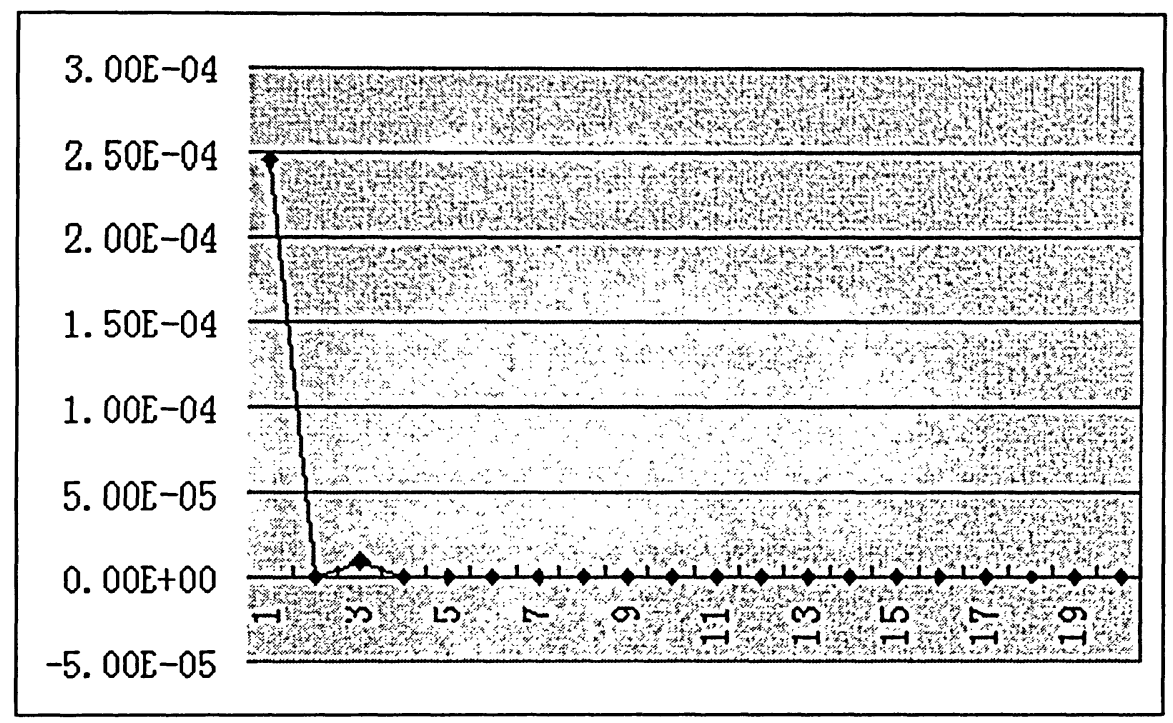

Figure 4-9 Coefficient differences between the refined and original RPCs of the $x$ numerator in the left image. 


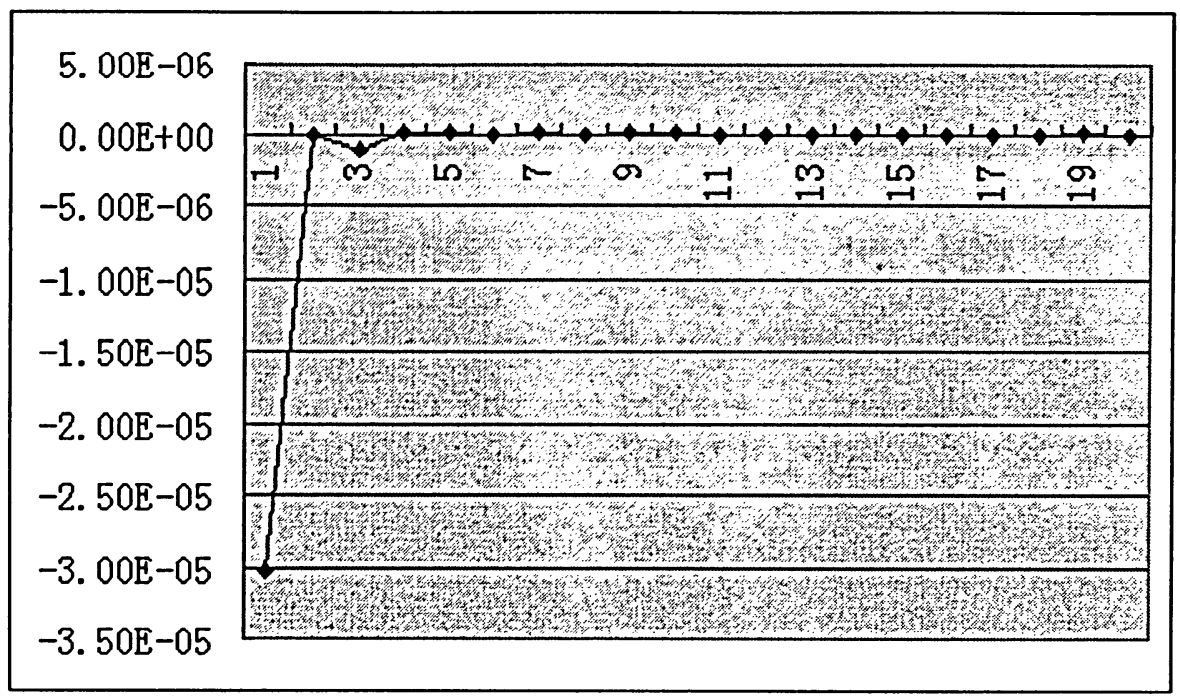

Figure 4-10 Coefficient differences between the refined and original RPCs of the $y$ numerator in the left image.

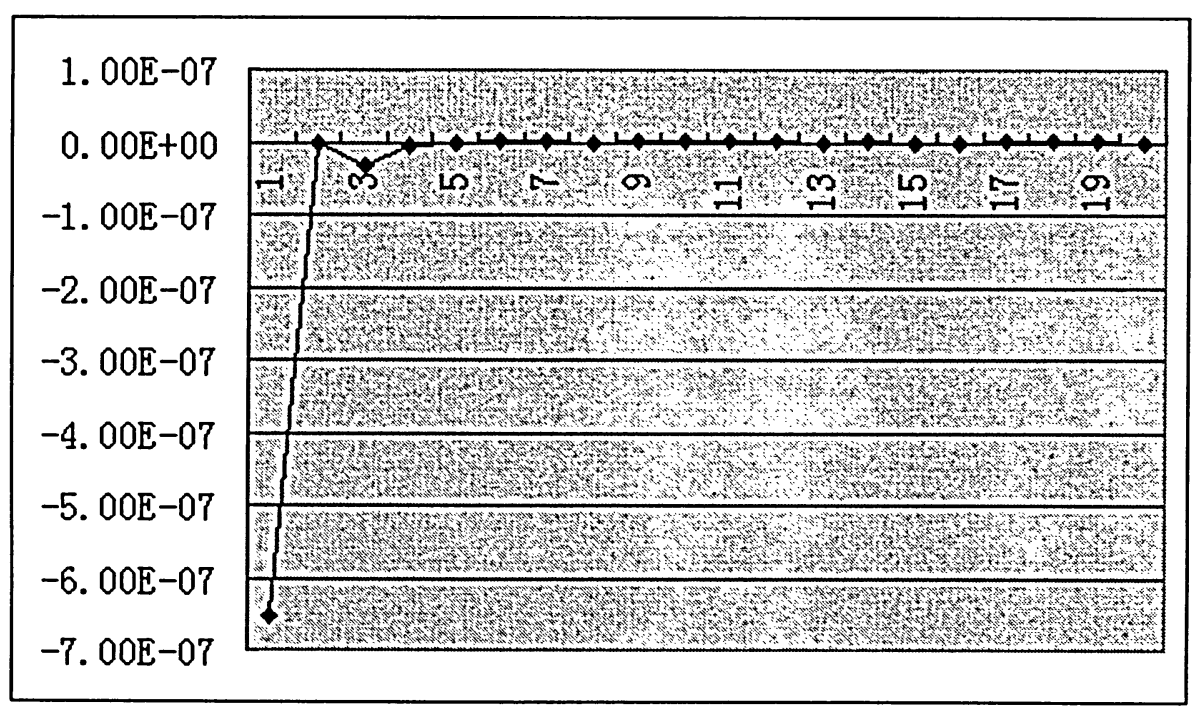

Figure 4-11 Coefficient differences between the refined and original RPCs of the $x$ numerator in the right image. 


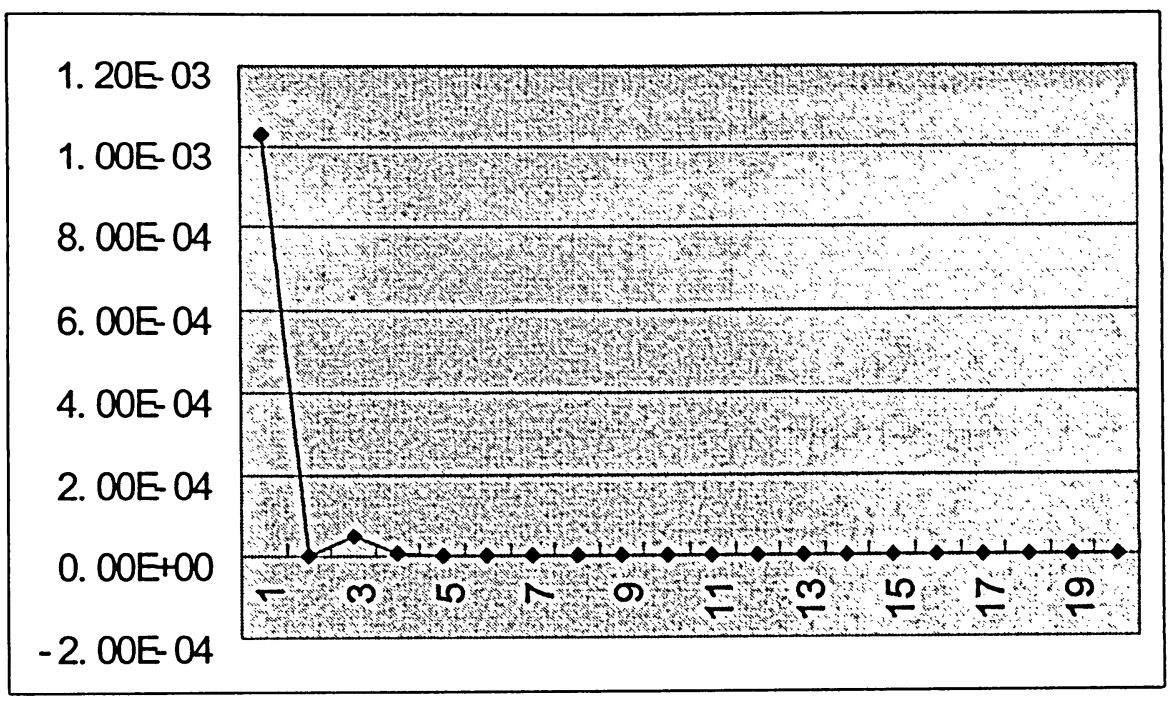

Figure 4-12 Coefficient differences between the refined and original RPCs of the $y$ numerator in the right image.

The similar experiment was also performed in the ground domain to find out in which domain the bias compensation could achieve a better result. In this experiment, one, three and four GCPs used in the previous tests were selected again. From the error vectors shown in Figures 4-13-4-15 and the RMSE values listed in Table 4-6, it is clear that the same accuracy level was achieved in ground domain. The main coefficients that contribute to the bias are $a_{0}, b_{0}$ and $c_{0}$, the effect of the other coefficients could be eliminated if there are a small number of GCPs available. The results are also not sensitive to the distribution of the GCPs as the case in image domain. The difference is that more coefficients are required to calculate in ground domain, which means more GCPs are needed. It is same when only the accuracy is considered during the biases compensation processing. But the bias compensation in image domain could be fulfilled by changing the RPCs only after the biases are calculated, which contains fewer calculations than the way in ground domain since the bias compensation has to be 
performed for each of the point after the $3 \mathrm{D}$ reconstruction.

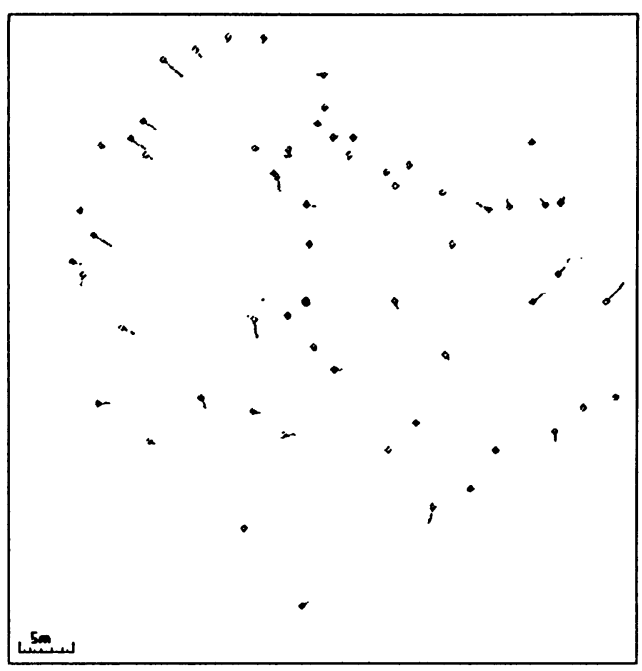

(a)

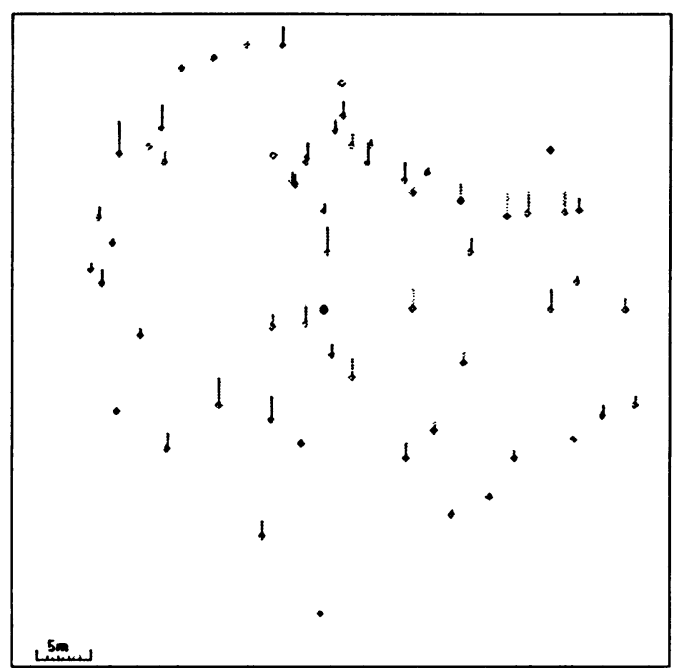

(b)

Figure 4-13 Error vectors in (a) planimetry and (b) elevation of ICPs using 1 GCP in the centre to remove the bias in ground domain.

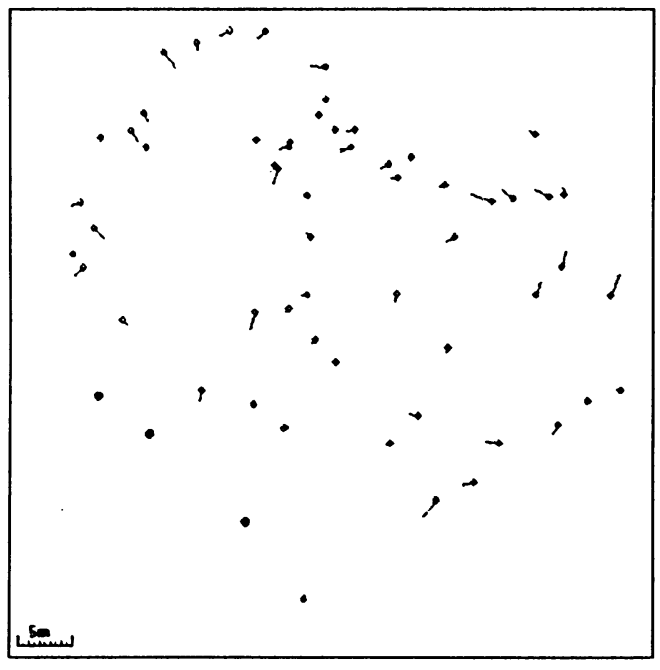

(a)

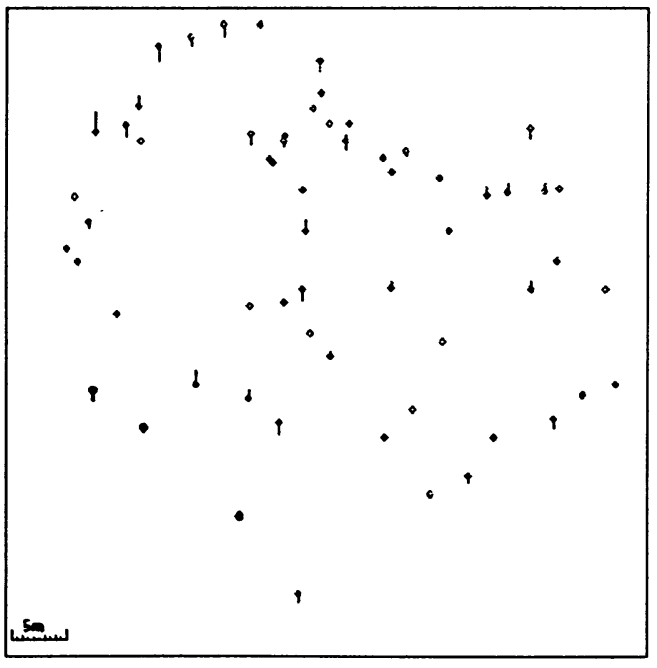

(b)

Figure 4-14 Error vectors in (a) planimetry and (b) elevation of ICPs using 3 GCPs at the edge to remove the bias in ground domain. 


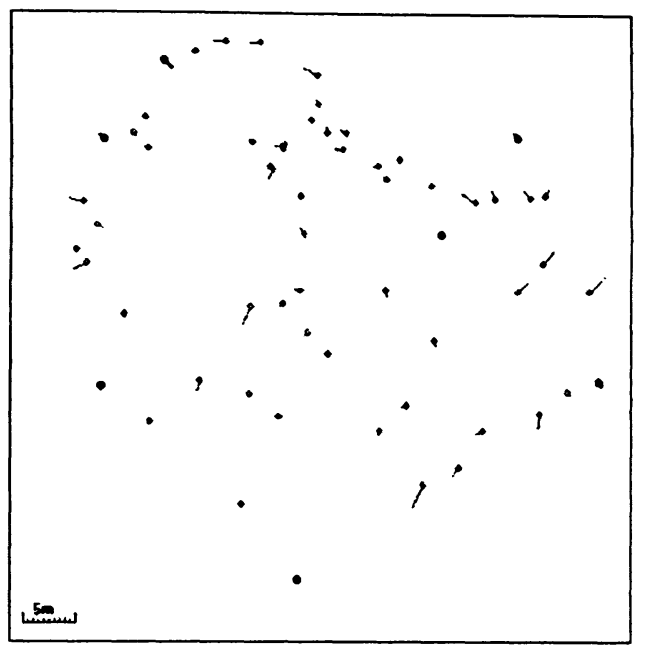

(a)

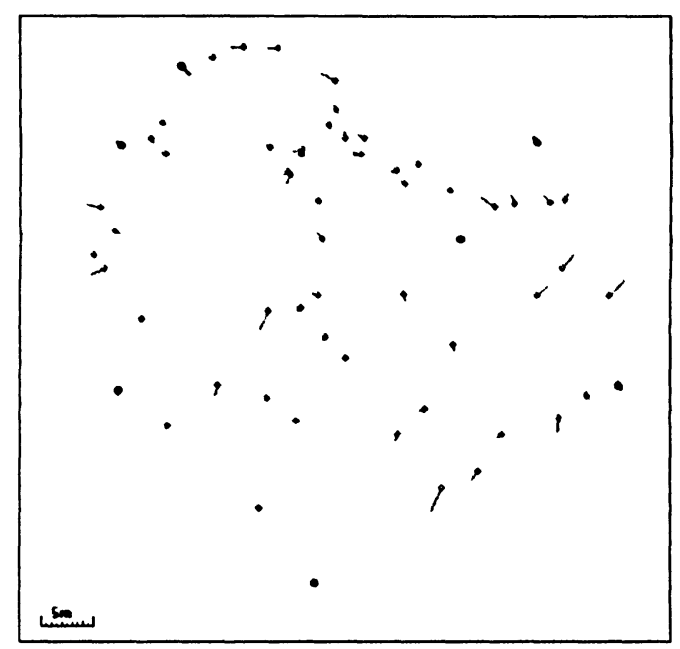

(b)

Figure 4-15 Error vectors in (a) planimetry and (b) elevation of ICPs using 7 GCPs at the corner to remove the bias in ground domain.

Table 4-6 RMSE of checkpoints using different selection of GCPs to remove the biases in ground domain

No. of

GCPs/ICPs

$1 / 62$

$3 / 60$
Model Used

$\mathbf{X}$

0.8

$$
L=b_{0}
$$$$
H=c_{0}
$$

\section{RMSE of Checkpoints (m)}

Y

Z

0.7

1.4

$$
\begin{aligned}
& P=a_{0}+a_{1} P_{R F}+a_{2} L_{R F} \quad 0.8 \quad 0.7 \\
& L=b_{0}+b_{1} P_{R F}+b_{2} L_{R F} \\
& H=c_{0}+c_{1} P_{R F}+c_{2} L_{R F}
\end{aligned}
$$


$7 / 56$

$$
\begin{aligned}
& P=a_{0}+a_{1} P_{R F}+a_{2} L_{R F}+a_{3} H_{R F} \\
& L=b_{0}+b_{1} P_{R F}+b_{2} L_{R F}+b_{3} H_{R F} \\
& H=c_{0}+c_{1} P_{R F}+c_{2} L_{R F}+c_{3} H_{R F}
\end{aligned}
$$$$
0.8
$$

0.7

\subsubsection{D Reconstruction using the 3D physical Model}

Another well-known mathematical model for 3D reconstruction of IKONOS imagery is the 3D physical model developed at the CCRS. This model has been integrated into the PCI Geomatica OrthoEngine software package. The math model calculates the position and orientation of the sensor at the time when the image was taken. As mentioned in chapter 3, the 3D physical model is based on the co-linearity condition, which represents the physical law of transformation between the image space and the ground space. It uses the principles related to photogrammetry, geodesy, and cartography (PCI Geomatics, 2001).

Several tests were performed to test the $3 \mathrm{D}$ reconstruction accuracy by using the $3 \mathrm{D}$ physical model. Six GCPs is the theoretical minimum for the 3D physical model. The use of redundant GCPs in the least-squares bundle adjustment reduced the effect of different input data errors. And these input errors are reflected in the residuals of the GCPs. Due to the large redundancy in the adjustments, the RMSE residuals of the GCPs are of the same order of magnitude as the input data errors. In this study, the number of GCPs used was $6,7,8,10,15,20,30,40,50$ and 60 to find the relationship between the 
number of the GCPs and the accuracy of the derived DEMs. The accuracy was evaluated by the RMSE values on the remaining ICPs. The selection of the GCPs used was based upon the best distribution of the GCPs. Figure 4-16 shows the relationship of the number of GCPs and the RMSE in the $\mathrm{X}$ and $\mathrm{Y}$ direction of the ICPs.

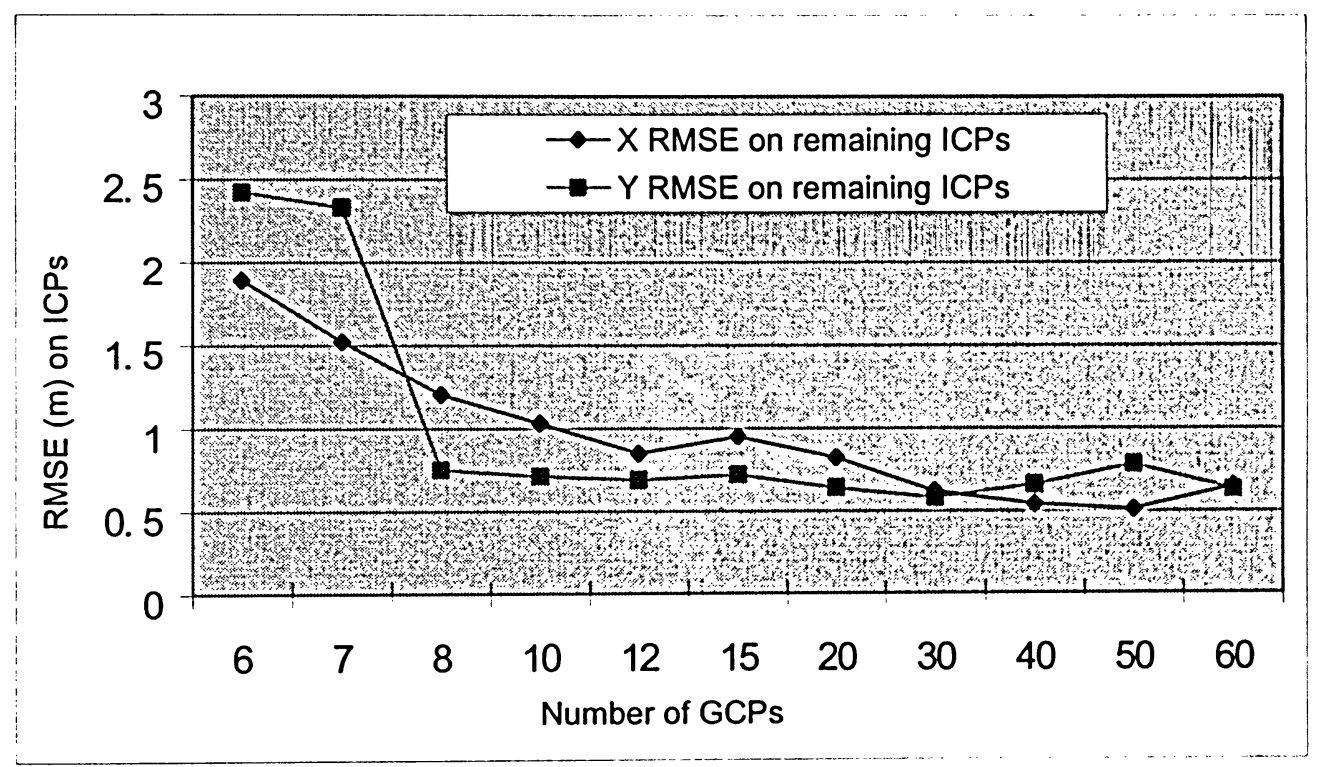

Figure 4-16 RMSE values for ICPs from the least-squares bundle adjustment computed with GCPs varying from 6-60 for the study area.

It can be seen clearly that when 12 GCPs are used, the RMSE values on both X and Y direction of the ICPs are in the order of submetre. There is no significant improvement if more GCPs used in the bundle adjustment. When 8-10 or less GCPs are used, the input errors propagated more into the $3 \mathrm{D}$ physical model because of a low degree of freedom in the least-squares adjustment. Consequently, 12 to 15 GCPs are a relative good comprise to maintain submetre level accuracy on the ICPs. So in this study, 12 well-distributed ground points were selected as the GCPs to perform the bundle 
adjustment and the other 51 points were used as ICPs. The RMSE of the GCPs residuals and the ICPs of this test are listed in Table 4-7. Figure 4-17 shows the distribution of the GCPs and ICPs. The GCPs are marked with black crosses and ICPs are marked with gray crosses.

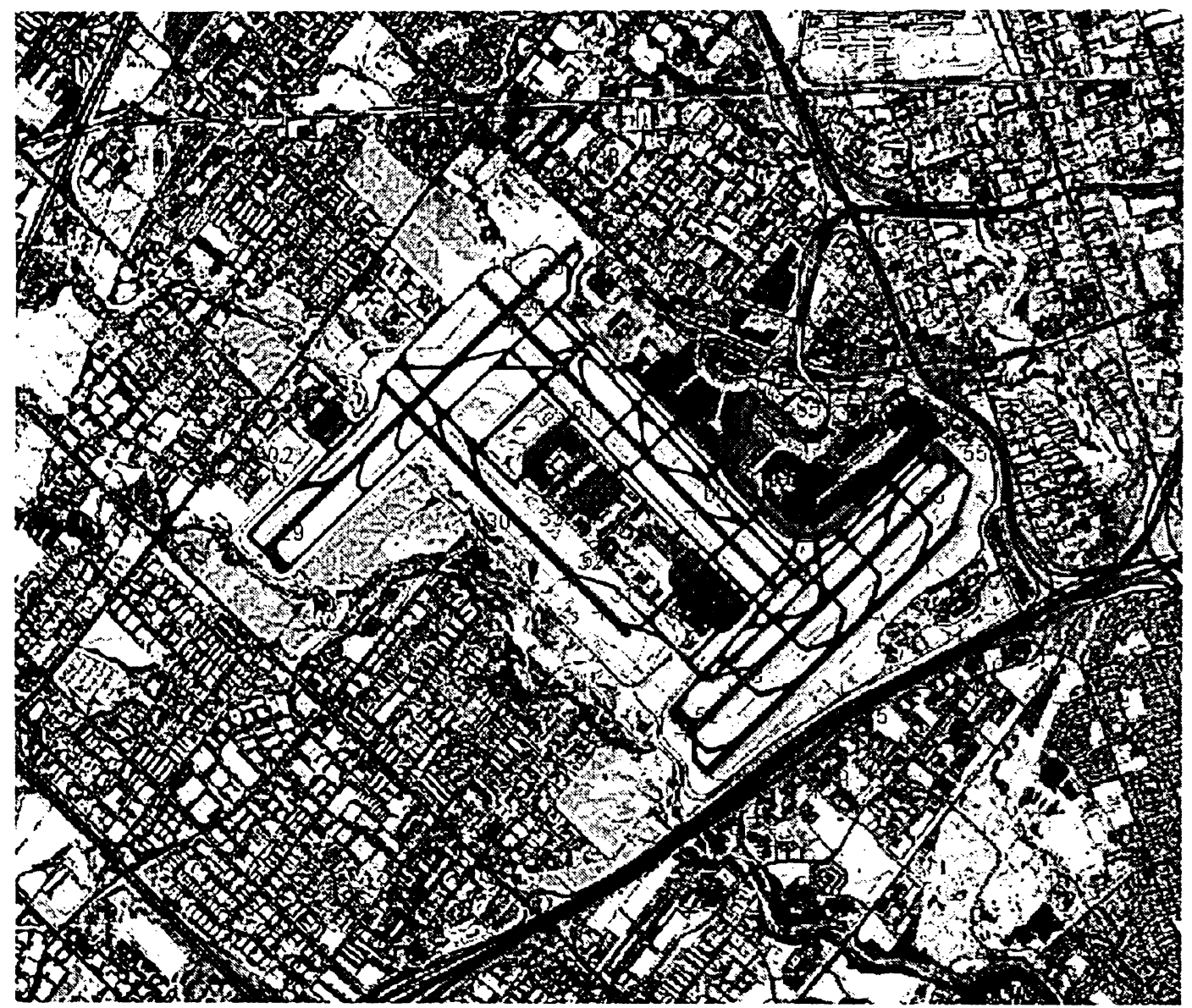

Figure 4-17 Distribution of the GCPs and ICPs used in the 3D physical model bundle adjustment. 
Table 4-7 RMSE, average and maximum errors of GCPs and ICPs calculated using the $3 \mathrm{D}$ physical model

\begin{tabular}{lcccccc}
\hline & \multicolumn{3}{c}{ GCPs Residuals (m) } & \multicolumn{3}{c}{ ICPs errors (m) } \\
& $\mathrm{X}$ & $\mathrm{Y}$ & $\mathrm{Z}$ & $\mathrm{X}$ & $\mathrm{Y}$ & $\mathrm{Z}$ \\
\hline RMSE & 0.4 & 0.5 & 0.5 & 0.9 & 0.7 & 0.7 \\
Average & -0.2 & 0.1 & 0.0 & 0.4 & -0.5 & -0.4 \\
Max & 1.0 & 1.0 & 1.4 & 2.6 & 3.3 & 1.5 \\
\hline
\end{tabular}

\subsection{Epipolar Images and DEM Generation}

The next step of DEM generation after the math model calculation is the epipolar images generation. The epipolar images are stereo pairs that are reprojected so that the left and right images have a common orientation, and matching features between the images appear along a common $x$ axis. Using epipolar images increased the speed of the correlation process and reduced the possibility of incorrect matches. The following two Figures 4-18 and 4-19 are the epiporlar projected image pair of the study area generated using PCI Geomatica OrthoEngine. 


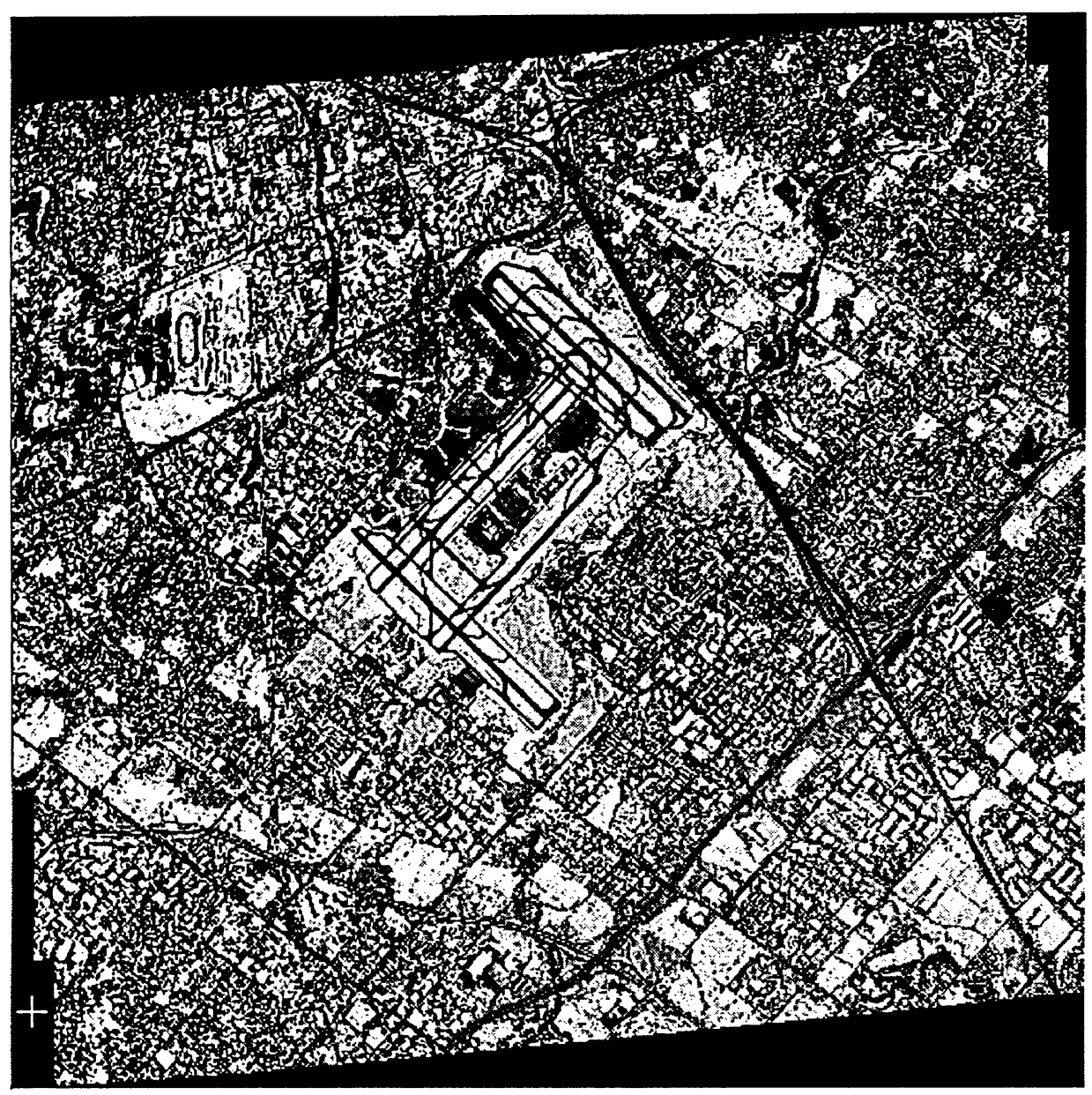

Figure 4-18 Left epiporlar image of the IKONOS in-track stereo images, north Toronto,

Ontario, Canada, 20 January 2004. 


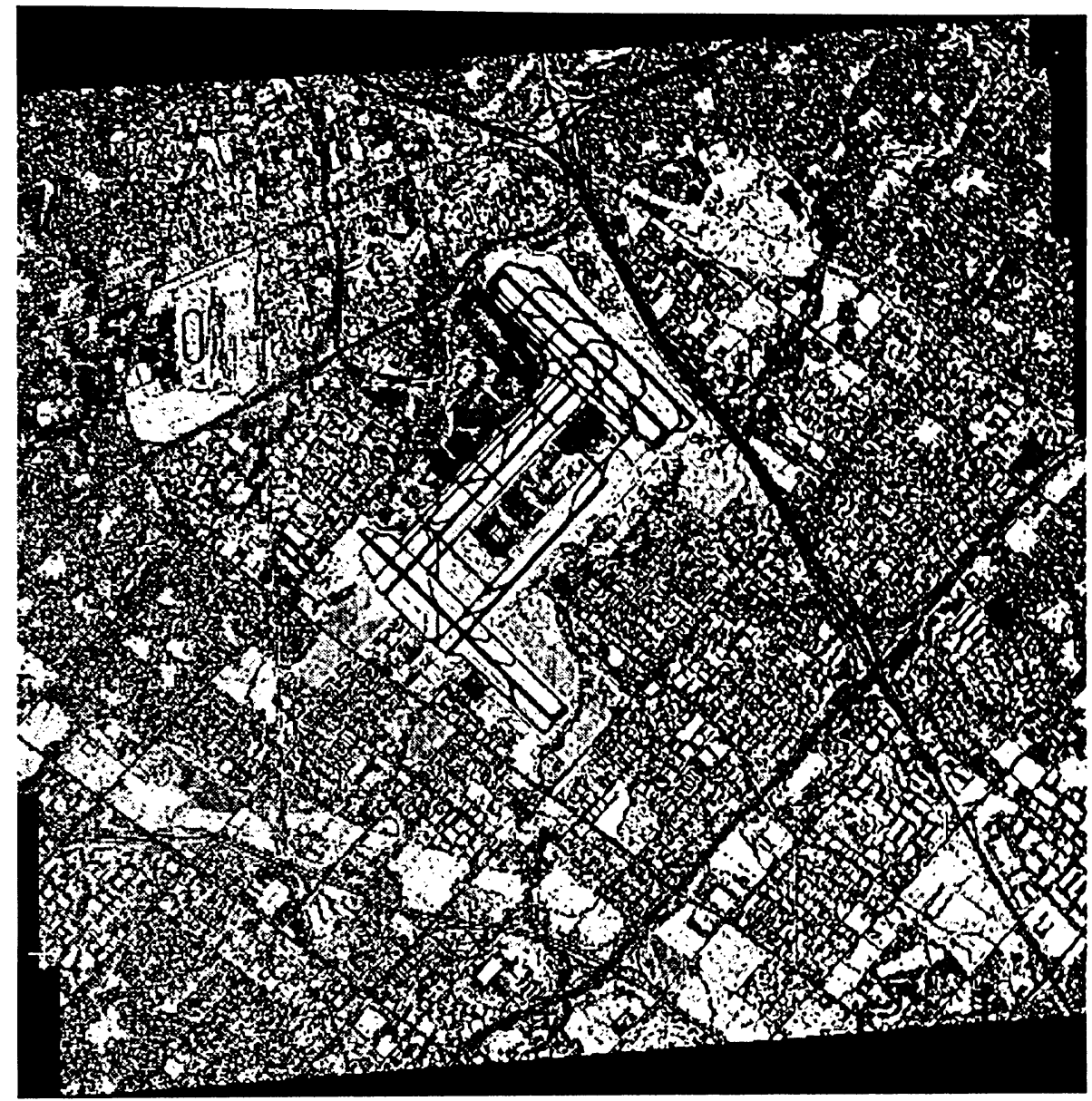

Figure 4-19 Right epiporlar image of the IKONOS in-track stereo images, north Toronto,

Ontario, Canada, 20 January 2004.

It can be seen from the epipolar pair that both images seem to be rotated $90^{\circ}$. This is due to the fact that the method used to find the conjugate points from the stereo pair used in PCI Geomatica OrthoEngine is along the $x$ axis. But the elevation parallax in the IKONOS in-track stereo images is mainly in $y$ direction. So the images are rotated in the epipolar image generation process to match the method of searching conjugate points along the $x$ axis direction.

After the epipolar image pair was generated, the next step is to find the conjugate points 
in the stereo pair and thus generate the epipolar DEM first. Figures 4-20 and 4-21 show the epipolar DEMs generated by using the refined RFM and the $3 \mathrm{D}$ physical model, respectively. The elevation at each point is described using the gray level of the pixel. The brighter the pixel, the higher of the elevation at that point. The elevation of the failed points is set to $-100 \mathrm{~m}$ and the elevation of the background is set to $-150 \mathrm{~m}$. Although the images are taken in winter time, the matching result is quite good. The percentage of successfully matched points is more than $99 \%$ in both cases of the refined RFM and the 3D physical model, which can also be seen from the epipolar DEMs showed below. The black dots are the failed matching area, which were given the elevation value of $-100 \mathrm{~m}$ and shown as black in the epipolar DEM image.

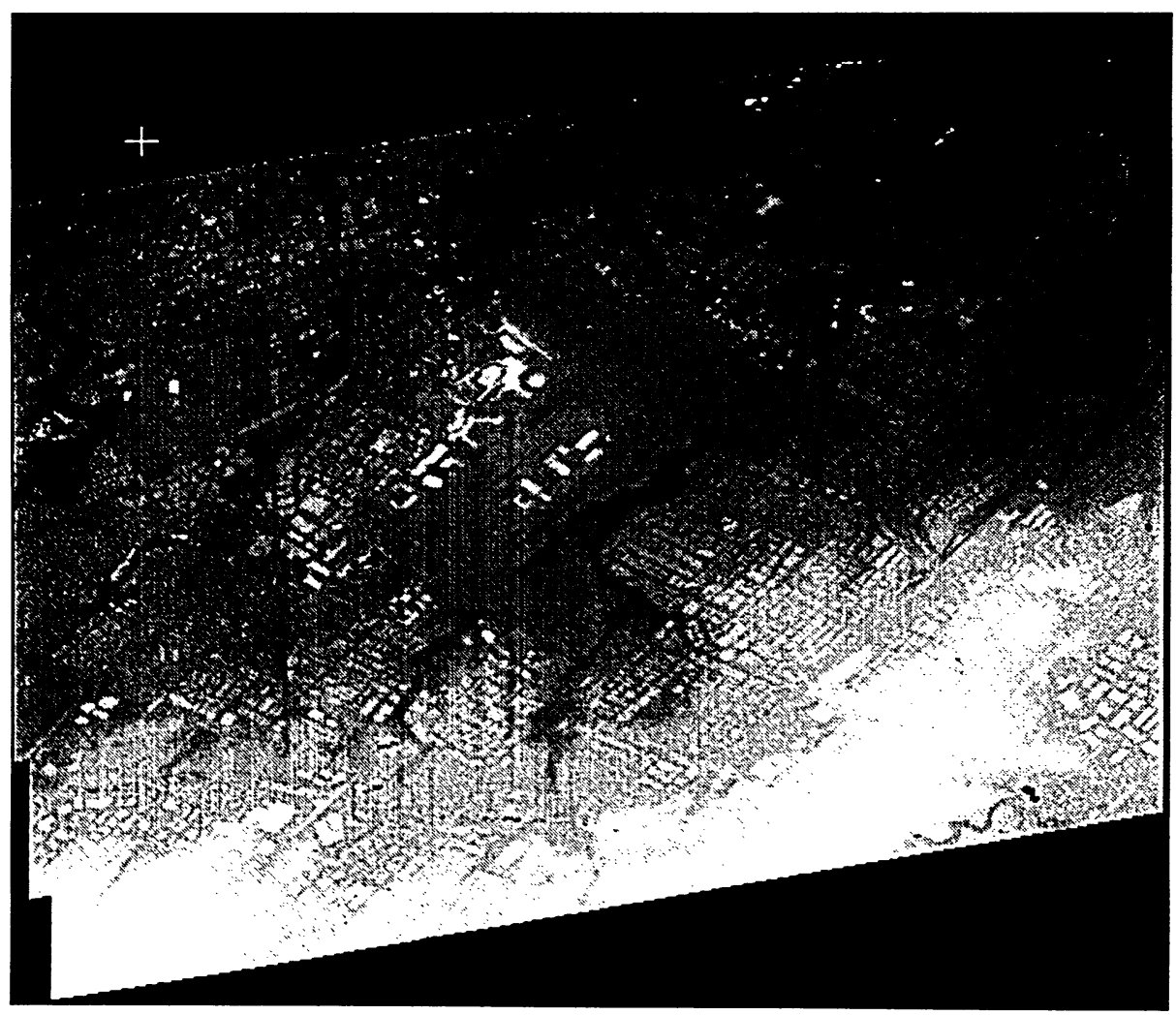

Figure 4-20 Epipolar DEM generated using the refined RFM. 


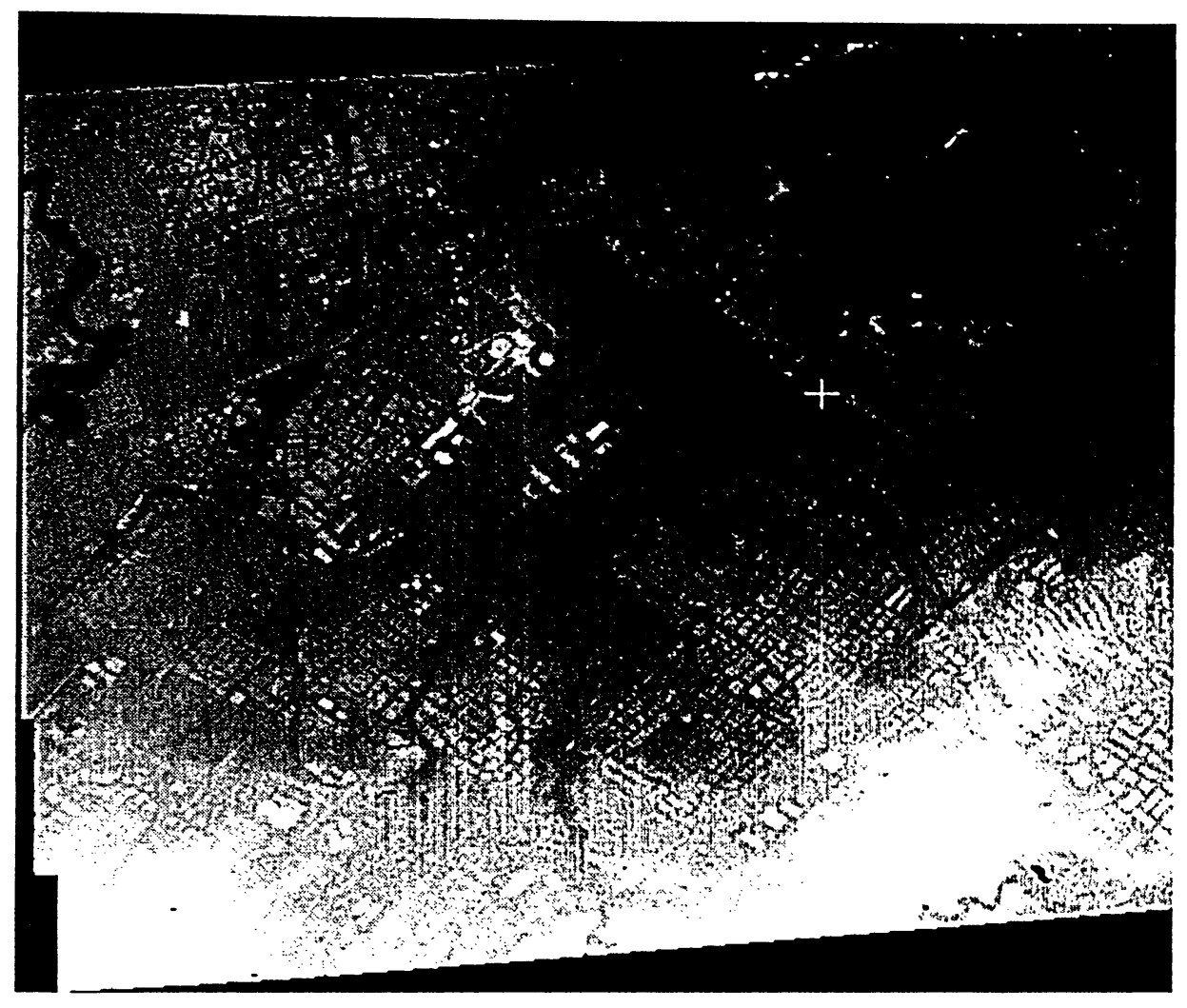

Figure 4-21 Epipolar DEM generated using the 3D physical model.

Based on the epipolar DEMs, the geocoded DEMs could be achieved. In this study, only the part under the control of the GCPs of the entire area was geocoded. The geocoded DEMs were generated by 2-pixel spacing with $3 \mathrm{~m}$ pixel resolution and the UTM projection, Zone 17 and NAD27 datum. The geo-coordinates of the DEM at the upper left corner are $606422.000 \mathrm{~N}, 4840119.000 \mathrm{E}$ and $614258.000 \mathrm{~N}, 4833084.000 \mathrm{E}$ at the lower right corner. The elevations are referenced to MSL. The size of the generated DEMs is 2612 pixels by 2345 pixels.

Figures 4-22 and 4-23 are the geocoded DEMs by using the refined RFM and the 3D physical model, respectively. Another DEM was also generated using the 
vendor-supplied RPCs, which is not shown in the following figures since the processing steps is similar to the DEM extracted using the refined RFM.

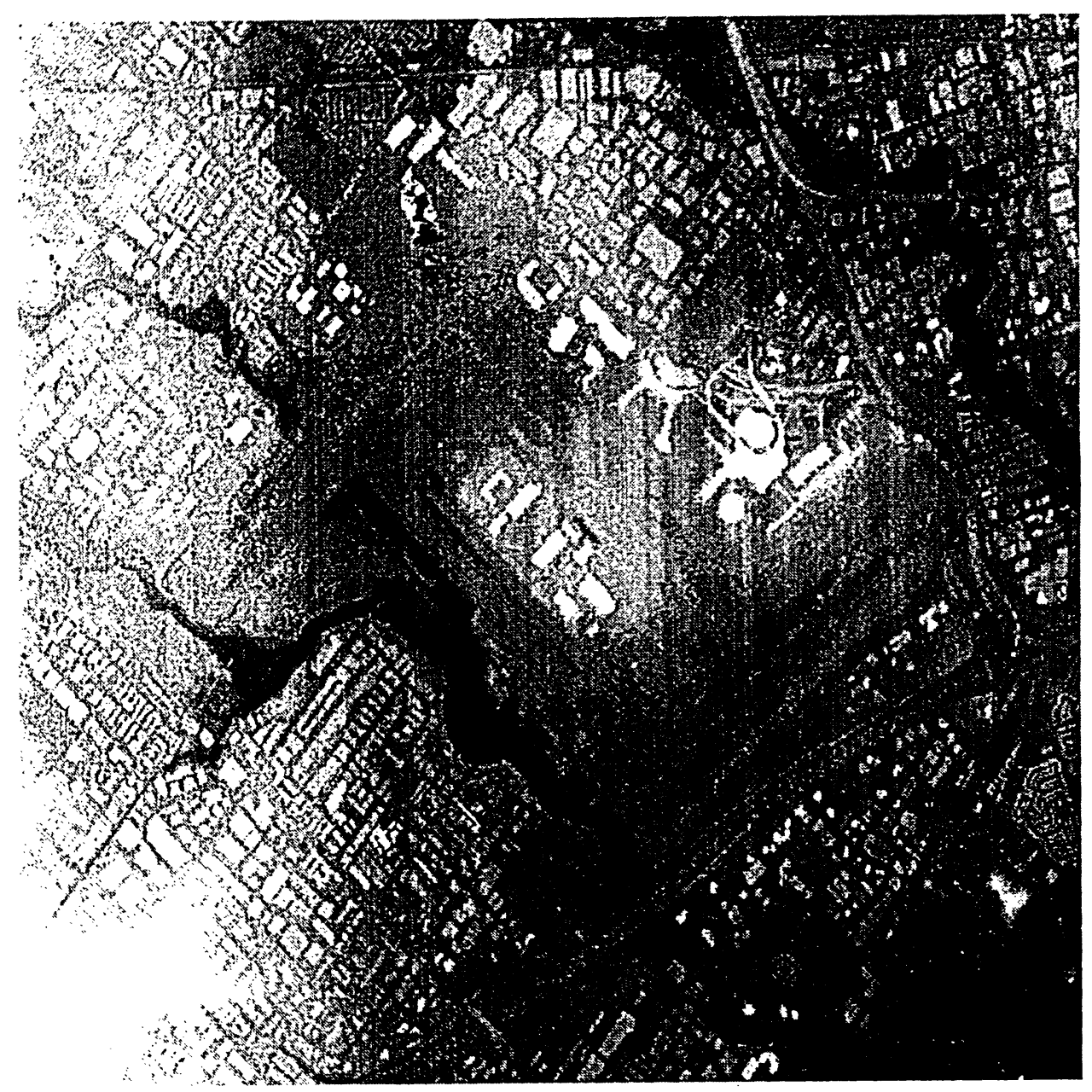

Figure 4-22 Geocoded DEM derived using the RFM model. 


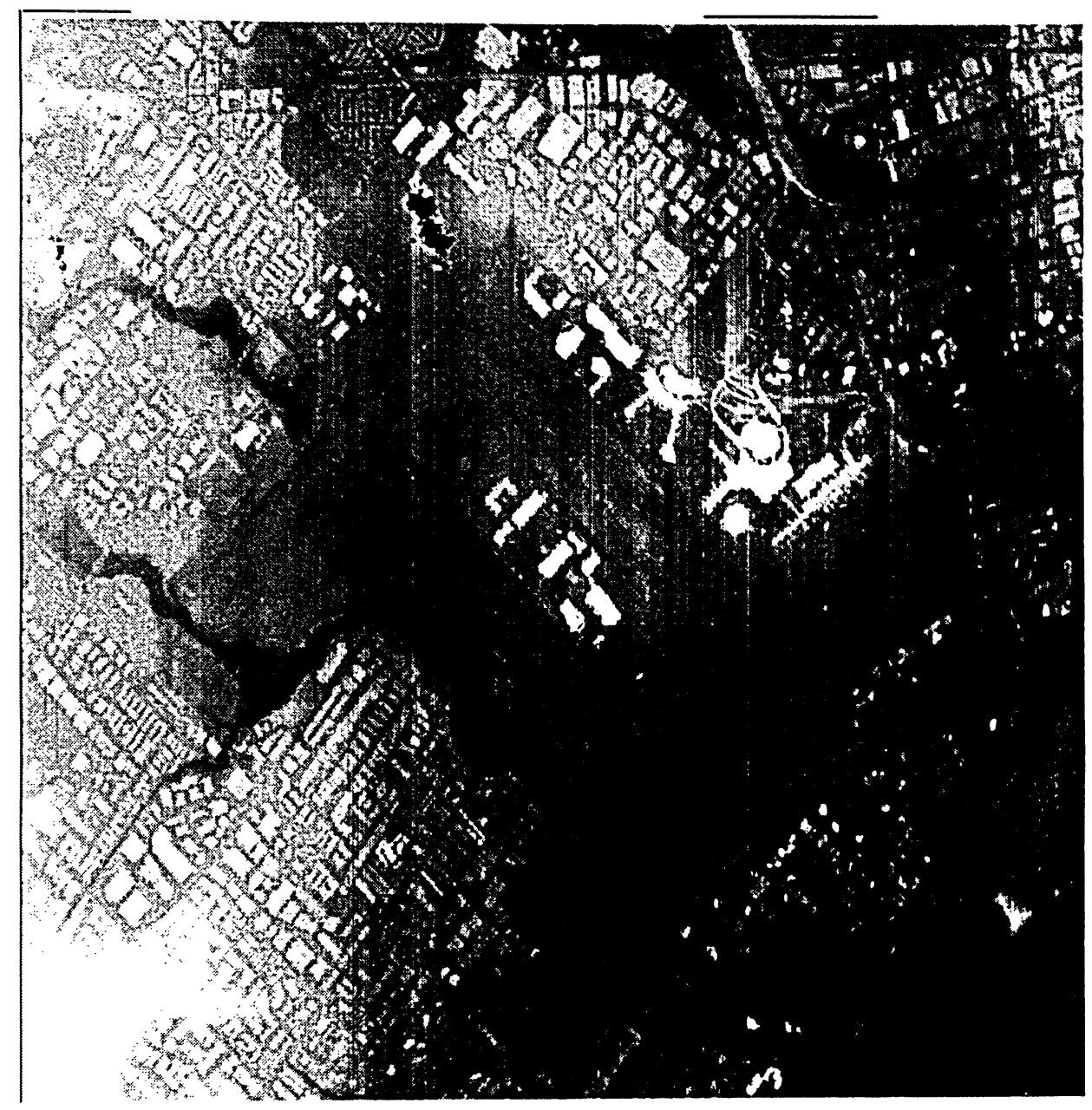

Figure 4-23 Geocoded DEM derived using the 3D physical model.

\subsection{DEM Editing}

From the geocoded DEMs shown in Figures 4-22 and 4-23, the derived DEMs look smooth except there are some mismatched areas. To fix the failed area and also to find some incorrect values, a series of post-editing has been done to the geocoded DEMs including the noise removal, the interpolation and the smoothing.

Noise refers to pixels containing distorted or failed values. Since pixels adjacent to 
failed matching points tend to contain incorrect values as well. The Noise Removal function in the PCI Geomatica OrthoEngine uses two filters to identify failed pixel values and their surrounding pixels. The first filter calculates the average and variance of the eight elevation values immediately surrounding each pixel, excluding failed and background pixels. If the center pixel is more than two standard deviations away from the average, it is replaced with the failed value. The second filter counts the number of failed values immediately surrounding each pixel. If five or more failed pixels border the center pixel, then the center pixel is also set to the failed value. Figure 4-24 shows the result of noise removal processing. There are some incorrect values have been set to failed values by using the two filters, which are marked with dark points in the DEM.

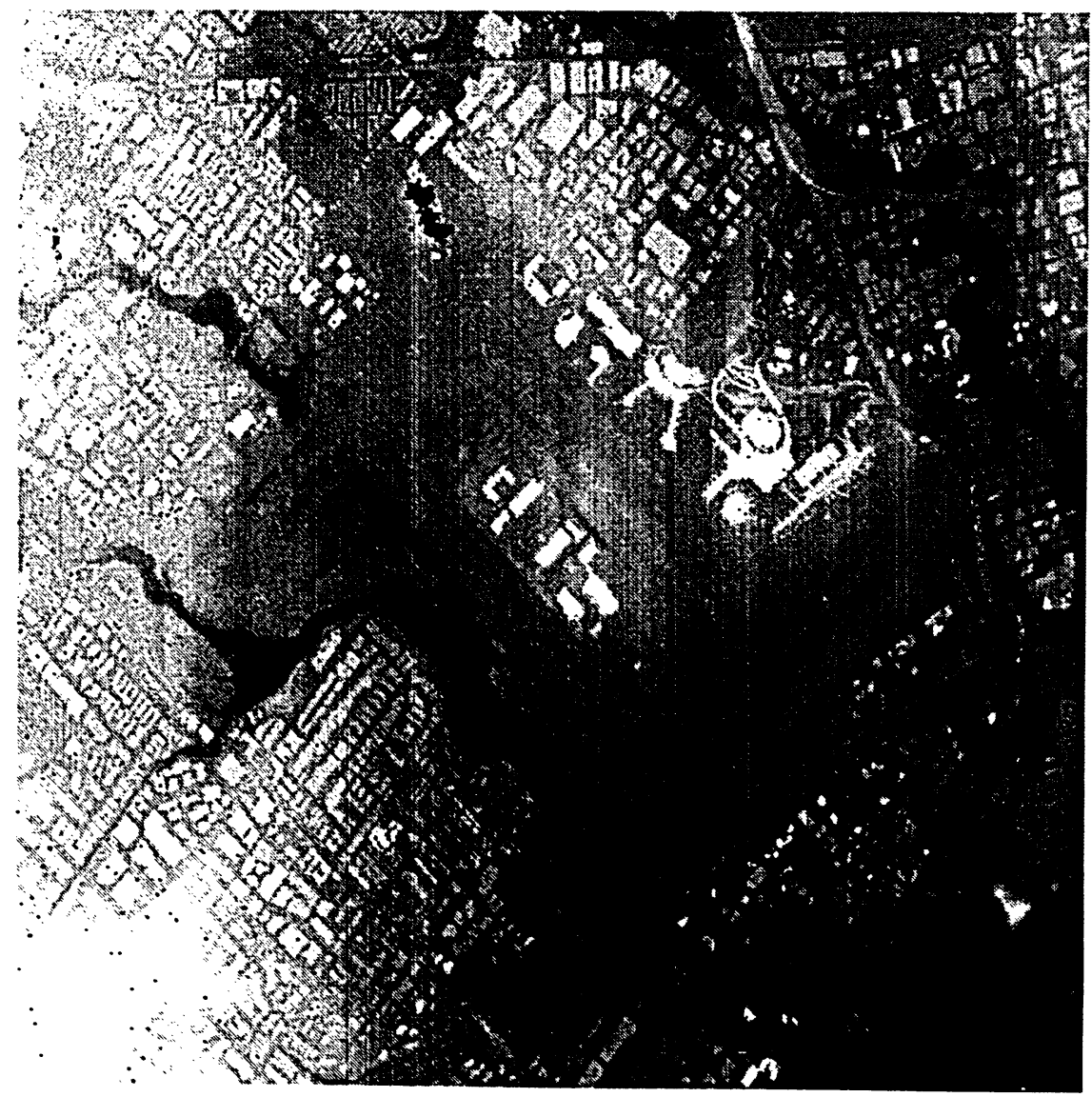

Figure 4-24 DEM derived using the 3D physical model after noise removal processing. 
The next step is to replace failed values with correct ones. Here the method of the interpolation was used. The interpolation filter replaces failed values with an estimate weighted by distance calculated from the valid pixels surrounding the failed pixels. Figure 4-25 shows the DEM after interpolation processing from the result of noise removal. The failed values found in the last step now have been replaced by the new values calculated from the surrounding pixels.

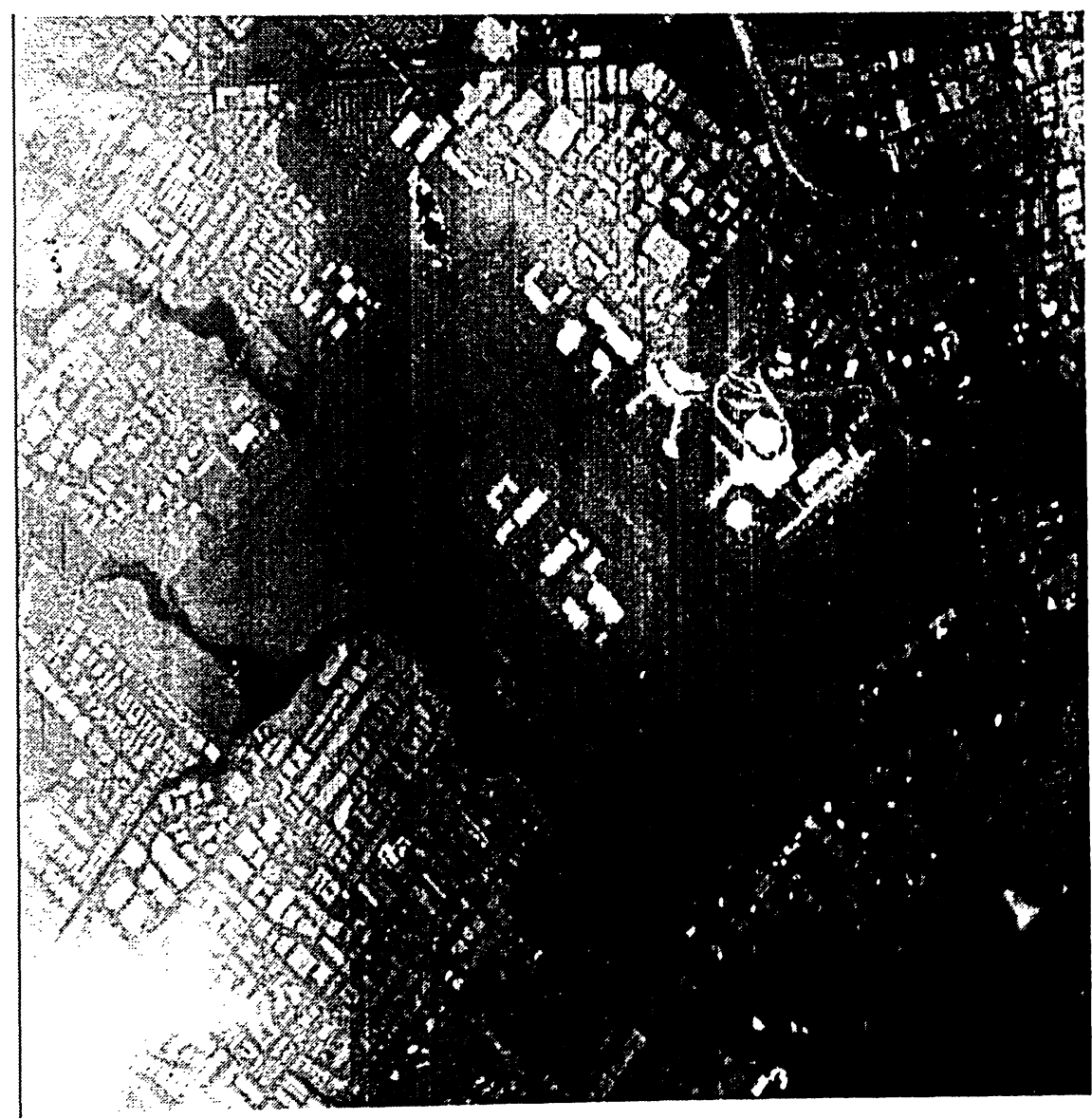

Figure 4-25 DEM derived using the 3D physical model after interpolation.

The smoothing process was then performed to the DEMs to produce a smooth result. The smooth filter used here is a Gaussian filter that calculates the weighted sum of all 
the pixels in a three by three pixel frame and assigns the value to the center pixel in the frame. Failed and background pixel values are not replaced by the filter and are not used in the Gaussian calculation. The result showed in the Figure 4-26 shows the DEM after smoothing processing.

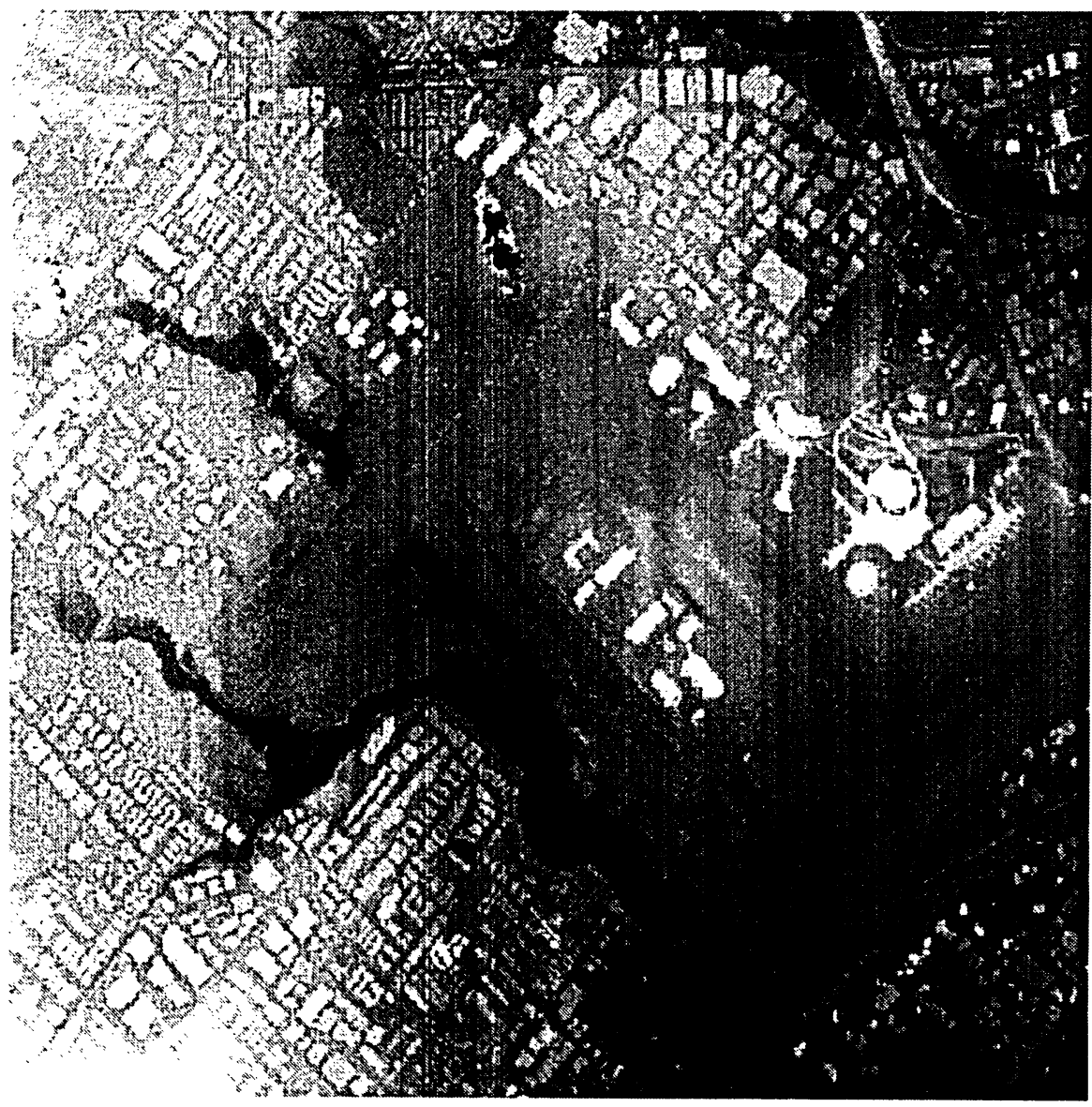

Figure 4-26 DEM derived using the 3D physical model after smoothing.

The edited result of the DEM generated from the Rational Function model is similar to the DEM from the 3D physical model. After the post editing of the geocoded DEM, the final DEM looks quite good, no failed points and the whole DEM is smooth. But to evaluate the quality of the generated DEM, some further evaluation of the accuracy is still needed. 


\subsection{Chapter Summary}

Based on the methods mentioned in chapter 3, five steps were performed in this chapter. The first step is the data preparation of study area. It includes acquisition and preprocessing of the IKONOS stereo data (involving the image pair and metadata including the RPC files) and the collection of GCPs from the orthoimage and DEM in the study area. The second step is 3D reconstruction using two different models (the RFM and the 3D physical model). For the RFM model, the biases in the vendor-supplied RPCs were calculated using GCPs and a new set of refined RPCs are then generated. For the 3D physical model, the influence of the required number of GCPs was discussed and a set of 12 GCPs with good distribution was selected to perform the $3 \mathrm{D}$ reconstruction in the $3 \mathrm{D}$ physical model. Then the $3 \mathrm{D}$ reconstruction accuracy of both of these two mathematical models was evaluated. The third step is the generation of DEM, which includes epipolar images generation, automatically conjugate points matching and DEM geocoding. The next step is DEM Editing. Through noise reduction, DEM interpolation and smoothing, most mismatched areas are fixed in this step. 


\section{DEM ACCURACY EVALUATION}

This chapter addresses the accuracy evaluation of the DEMs generated from IKONOS in-track stereo images. The evaluation of the DEMs in this study includes three parts. The first is to find the errors of the elevations in the geocoded DEMs on both ICPs and GCPs (see Section 5.1). The second is to find the difference between the DEMs extracted using the 3D physical model and the RFM with vendor-supplied and refined RPCs, respectively (see Section 5.2). The third is to compare the generated DEMs with the "ground truth" DEM that is manually edited from aerial photographs, this DEM is also used for the GCPs elevation collection. The different types of DEMs are compared in a desktop ERDAS Imagine V8.7 environment using its Spatial Modeler function (sec Section 5.3). Before such comparisons, the elevation reference datum of the DEMs extracted using the RFM has to be converted from ellipsoid to MSL and all the derived DEMs should be exported in the ERDAS "*.img” format, so that the Spatial Modeler of the ERDAS Imagine V8.7 could be used for DEM accuracy evaluation.

\subsection{Elevation Errors on ICPs and GCPs}

The elevation errors of the geocoded DEMs on both GCPs and ICPs, which are calculated using the RFM with the vendor-supplied RPCs and the refined RPCs, and the 3D physical model, respectively, are listed in Table 5-1. The GCPs are marked in gray since different methods using different sets of GCPs. 
Table 5-1 Comparison of elevation differences using different 3D stereo models.

\begin{tabular}{|c|c|c|c|}
\hline $\begin{array}{c}\text { Point } \\
\text { Number }\end{array}$ & $\begin{array}{c}\text { Elevation } \\
\text { Difference using } \\
\text { the vendor-supplied } \\
\text { RPCs }(\mathrm{m}) \\
\end{array}$ & $\begin{array}{c}\text { Elevation } \\
\text { Difference } \\
\text { using the refined } \\
\text { RPCs }(\mathrm{m}) \\
\end{array}$ & $\begin{array}{c}\text { Elevation } \\
\text { Difference using } \\
\text { the } 3 \mathrm{D} \text { physical } \\
\text { model (m) }\end{array}$ \\
\hline 1 & -11.5 & 0.2 & 0.1 \\
\hline 2 & -12.0 & 0.0 & 0.0 \\
\hline 3 & -11.5 & -0.2 & 0.4 \\
\hline 4 & -12.1 & -0.2 & 0.4 \\
\hline 5 & -11.5 & 0.3 & -0.5 \\
\hline 6 & -11.1 & 0.6 & -0.7 \\
\hline 7 & -11.0 & 0.3 & -0.1 \\
\hline 8 & -11.2 & 0.6 & -0.1 \\
\hline 9 & -9.8 & 2.2 & 1.0 \\
\hline 11 & -10.8 & 0.8 & 0.3 \\
\hline 12 & -11.8 & 0.1 & 0.4 \\
\hline 13 & -11.1 & 0.8 & 0.7 \\
\hline 14 & -11.2 & 0.8 & 0.5 \\
\hline 15 & -10.7 & 0.6 & 0.4 \\
\hline 16 & -10.7 & 0.7 & 0.0 \\
\hline 17 & -10.9 & 1.5 & -0.3 \\
\hline 18 & -11.2 & 0.2 & -0.3 \\
\hline 19 & -11.5 & -0.2 & -0.6 \\
\hline 20 & -11.1 & -0.1 & -0.6 \\
\hline 21 & -11.5 & 0.2 & 0.0 \\
\hline 22 & -11.1 & 0.4 & 0.2 \\
\hline 23 & -10.7 & 0.8 & 0.1 \\
\hline 24 & -10.6 & 0.9 & 1.6 \\
\hline 25 & -9.7 & 2.1 & -0.1 \\
\hline 26 & -10.9 & 1.1 & -0.6 \\
\hline 27 & -11.3 & 0.4 & -0.5 \\
\hline 28 & -11.6 & 0.2 & -0.8 \\
\hline 29 & -11.4 & 0.3 & -0.4 \\
\hline 30 & -10.8 & 0.3 & -0.9 \\
\hline 31 & -11.1 & 0.6 & -1.3 \\
\hline 32 & -11.0 & 0.4 & -1.5 \\
\hline 33 & -10.8 & 0.8 & -0.4 \\
\hline 34 & -10.7 & 1.3 & -1.4 \\
\hline 35 & -11.6 & 0.0 & -0.4 \\
\hline 36 & -10.9 & 0.4 & -0.7 \\
\hline 37 & -11.3 & 0.2 & -0.3 \\
\hline 38 & -11.2 & 0.4 & 0.1 \\
\hline 39 & -11.4 & -0.1 & 0.1 \\
\hline 40 & -11.3 & 0.3 & 0.1 \\
\hline 41 & -10.6 & 0.5 & 0.7 \\
\hline 42 & -10.4 & 0.8 & 0.3 \\
\hline 43 & -11.7 & 0.2 & -0.5 \\
\hline 44 & -11.5 & -0.1 & 0.2 \\
\hline 45 & -12.1 & -0.2 & -0.4 \\
\hline 46 & -12.0 & -0.2 & -0.2 \\
\hline 47 & -12.3 & -0.3 & 0.0 \\
\hline 49 & -11.7 & -0.1 & 1.0 \\
\hline
\end{tabular}




\begin{tabular}{|c|c|c|c|}
\hline 50 & -12.2 & 0.1 & 0.1 \\
\hline 51 & -11.1 & 0.5 & -0.6 \\
\hline 52 & -11.5 & 0.1 & -1.5 \\
\hline 53 & -11.7 & 0.3 & -1.1 \\
\hline 54 & -11.7 & 0.0 & -1.3 \\
\hline 55 & -12.0 & -0.5 & 0.0 \\
\hline 56 & -11.6 & 0.1 & -0.7 \\
\hline 57 & -11.6 & 0.5 & -0.3 \\
\hline 58 & -12.0 & 0.1 & -0.7 \\
\hline 59 & -11.0 & 0.7 & -1.2 \\
\hline 60 & -11.0 & 0.6 & -1.2 \\
\hline 61 & -11.5 & 0.8 & -0.7 \\
\hline 62 & -11.4 & -0.2 & -0.1 \\
\hline 63 & -11.4 & 0.5 & 0.1 \\
\hline 64 & -11.2 & 0.5 & -0.6 \\
\hline 65 & -11.0 & 0.8 & 0.6 \\
\hline $\begin{array}{c}\text { RMSE } \\
\text { on ICPs }\end{array}$ & 11.3 & 0.7 & 0.7 \\
\hline Max & & & \\
$\begin{array}{c}\text { Error on } \\
\text { ICPs }\end{array}$ & -12.3 & 2.2 & 1.5 \\
\hline $\begin{array}{c}\text { Average } \\
\text { Error on } \\
\text { ICPs }\end{array}$ & & & -0.3 \\
\hline
\end{tabular}

As shown in Table 5-1, the results have been improved greatly by using the refined RPCs instead of the vendor-supplied one in the RFM. The 3D physical model and the RFM with refined RPCs could achieve the same accuracy level ICPs. But the RFM requires less number of GCPs as mentioned in Section 4.2.1.

\subsection{Comparison of the DEMs Extracted Using Different Sensor Models}

By calculating the elevation difference between each point in the DEMs, the difference DEM were then formed using ERDAS Imagine V8.7 Spatial Modeler function. The processing strategy of this comparison method is shown in Figure 5-1. Figure 5-2 shows the difference DEM by comparing the DEMs from the RFM with vendor-supplied RPCs and the 3D physical model. Figure 5-3 shows the difference DEM from between the 
DEM derived using the refined RFM and the DEM derived using the 3D physical model.

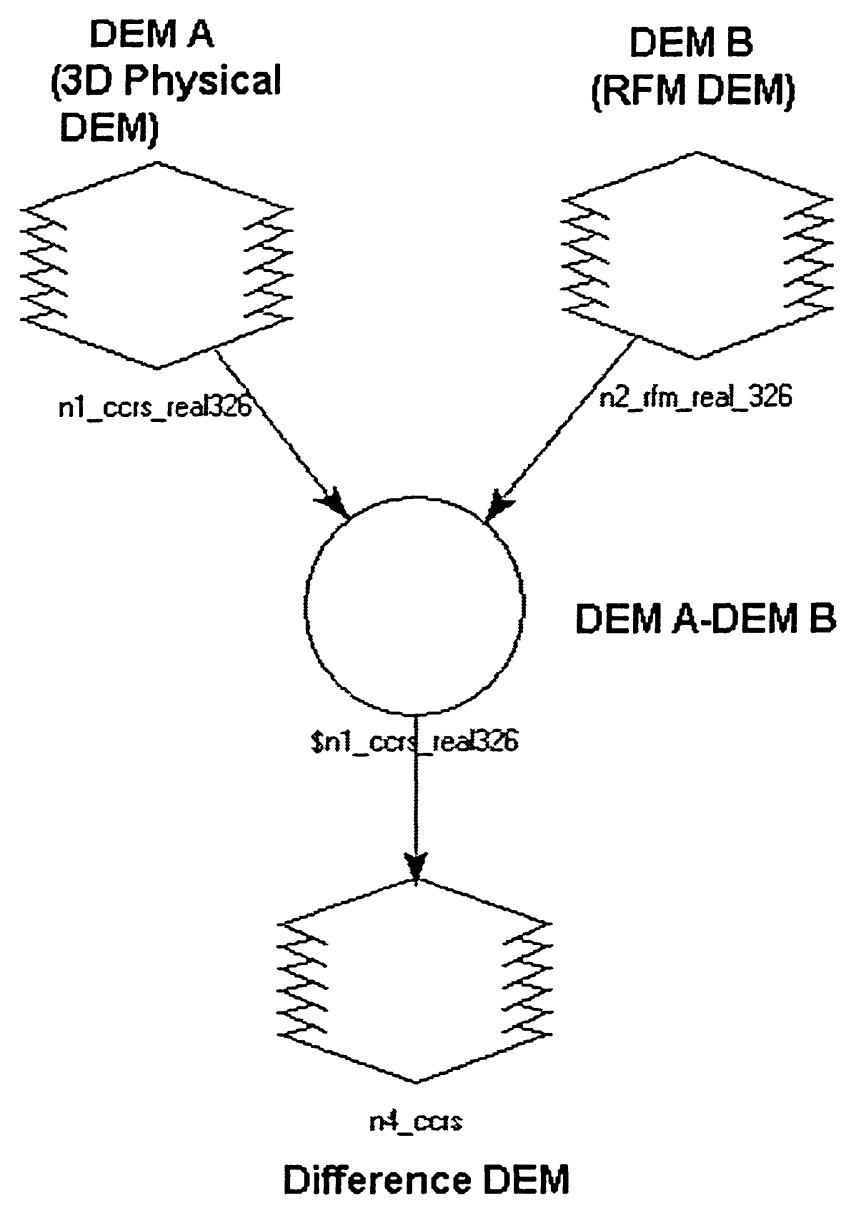

Figure 5-1 Processing strategy of the generation of difference DEM using ERDAS Imagine V8.7 Spatial Modeler. 


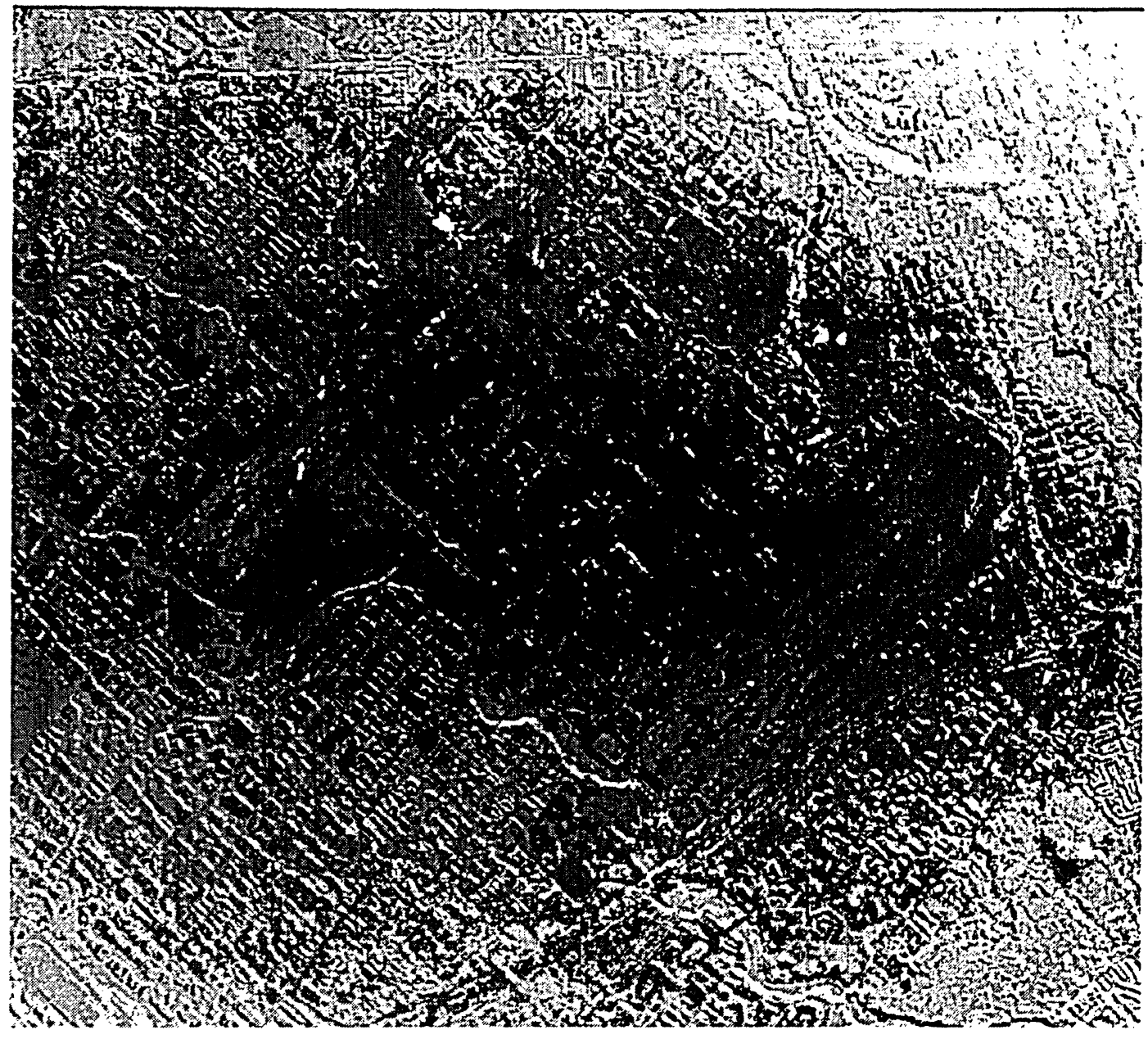

Figure 5-2 Difference DEM between the DEM extracted using the RFM with vendorsupplied RPCs and the DEM extracted using the 3D physical model. 


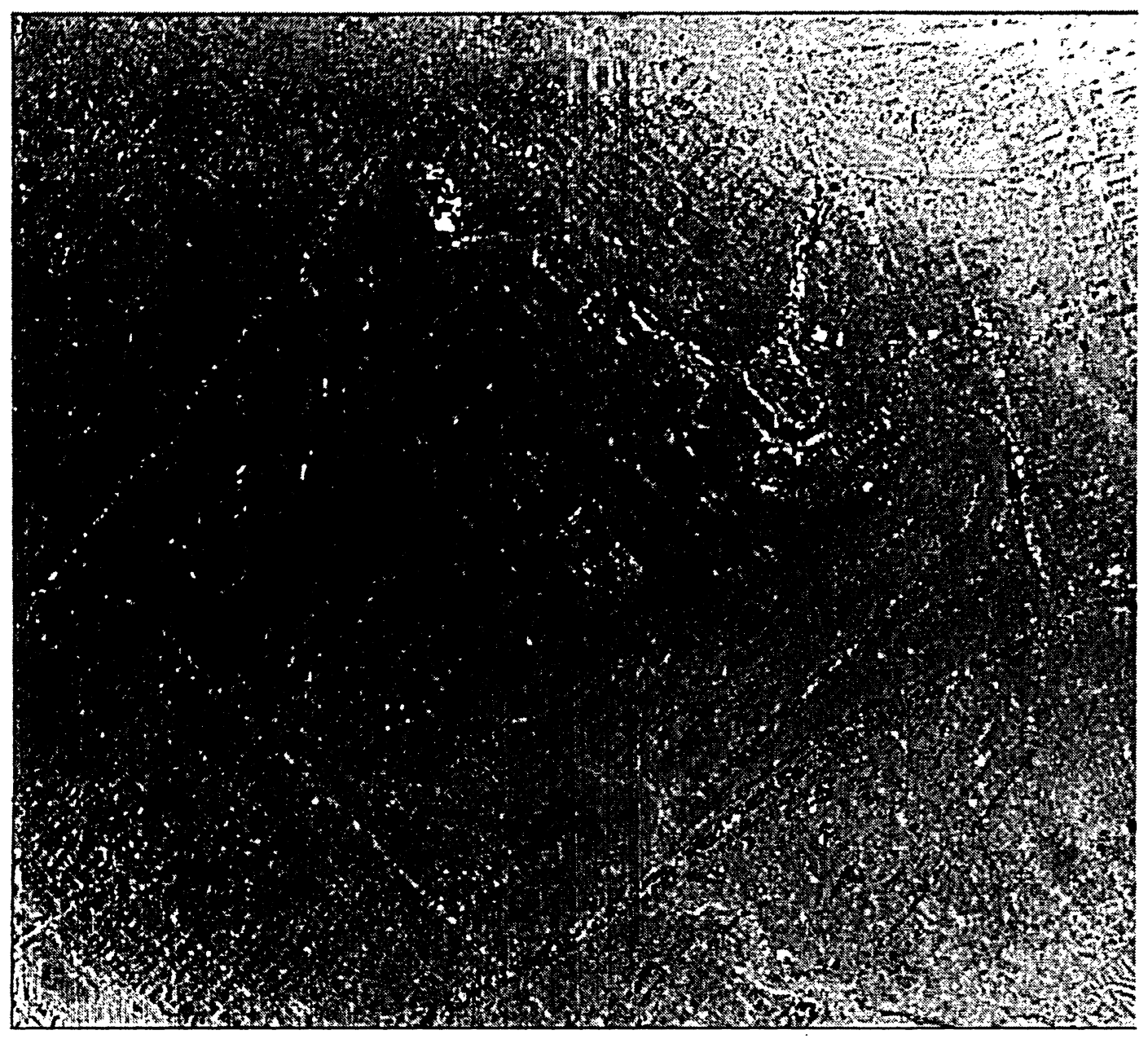

Figure 5-3 Difference DEM between the DEM extracted using the refined RFM and the DEM extracted using the $3 \mathrm{D}$ physical model.

It can be seen that the elevation difference distribute evenly and some important statistic results of the difference DEMs are listed in Table 5-2. A total of 6,125,140 points were used in the statistical analysis of the entire difference DEM. 
Table 5-2 Statistic results of the two sets of difference DEMs on ICPs

\begin{tabular}{|l|c|c|c|c|c|c|}
\hline $\begin{array}{c}\text { Source of the } \\
\text { Difference DEM }\end{array}$ & $\begin{array}{c}\text { Min } \\
(\mathrm{m})\end{array}$ & $\begin{array}{c}\text { Max } \\
(\mathrm{m})\end{array}$ & $\begin{array}{c}\text { Median } \\
(\mathrm{m})\end{array}$ & $\begin{array}{c}\text { Mode } \\
(\mathrm{m})\end{array}$ & $\begin{array}{c}\text { Mean } \\
(\mathrm{m})\end{array}$ & $\begin{array}{c}\text { STDE } \\
(\mathrm{m})\end{array}$ \\
\hline $\begin{array}{l}\text { 3D Physical Model } \\
\text { "minus" RFM (with } \\
\text { vendor-supplied } \\
\text { RPCs) }\end{array}$ & -15.5 & 37.8 & 11.2 & 10.8 & 11.5 & 1.9 \\
\hline $\begin{array}{c}\text { 3D Physical Model } \\
\text { "minus" RFM } \\
\text { (with refined RPCs) }\end{array}$ & -31.7 & 24.8 & -1.6 & -1.9 & -1.0 & 1.5 \\
\hline
\end{tabular}

As shown in Table 5-2, the DEM extracted using the RFM with the refined RPCs and the DEM extracted using the 3D physical model demonstrate a very good consistency at individual DEM grid points, as most of the DEM differences on ICPs are less than $2 \mathrm{~m}$. The larger elevation differences occur only at a few points when comparing with the number of points used in the statistical analysis. The distributions of the elevations in the difference DEMs are shown in Figures 5-4 and 5-5. As illustrated in Figure 5-4, most of the elevation differences $(6,067,262$ points) range from $-4.9 \mathrm{~m}$ to $2.6 \mathrm{~m}$, counting up to $99.06 \%$ of the elevation points selected from the entire DEM. As mentioned in Section 4.3, the percentage of the successfully matched points using these two mathematical models are more than $99 \%$, the points with large elevation difference could mainly be the mismatched points. The case in the second set of difference DEM is similar to the first set. Since some systematic errors are contained in the vender-supplied RPCs, elevation differences in this case range from $7.9 \mathrm{~m}$ to $17.9 \mathrm{~m}$ (see Figure 5-5), counting up to $99.05 \%$. Most of the values are around $11 \mathrm{~m}$, which is of the same magnitude order of the systematic error in elevations mentioned in Section 4.2.1. From the analysis of these two sets of difference DEMs, it could be further proved that the 
biases in the vendor-supplied RPCs have been compensated very well to the entire DEM with the method mentioned in Section 3.4.1.

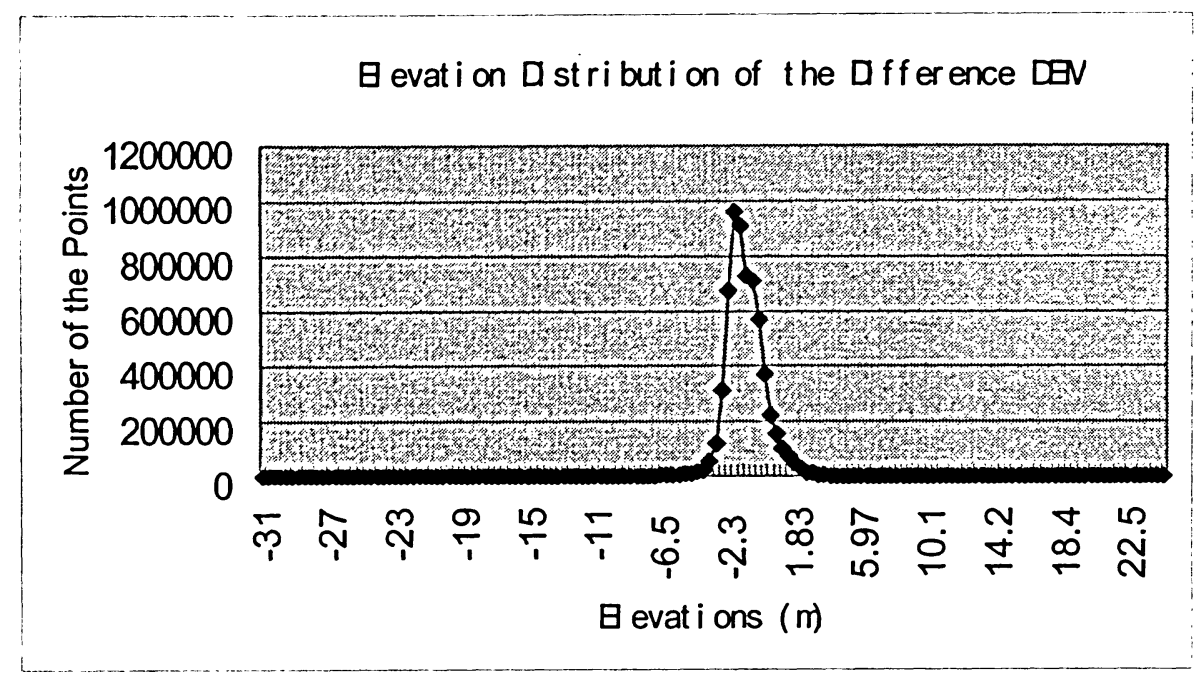

Figure 5-4 Elevation distribution of the difference DEM between the DEM derived using the 3D physical model and the DEM derived using the refined RFM.

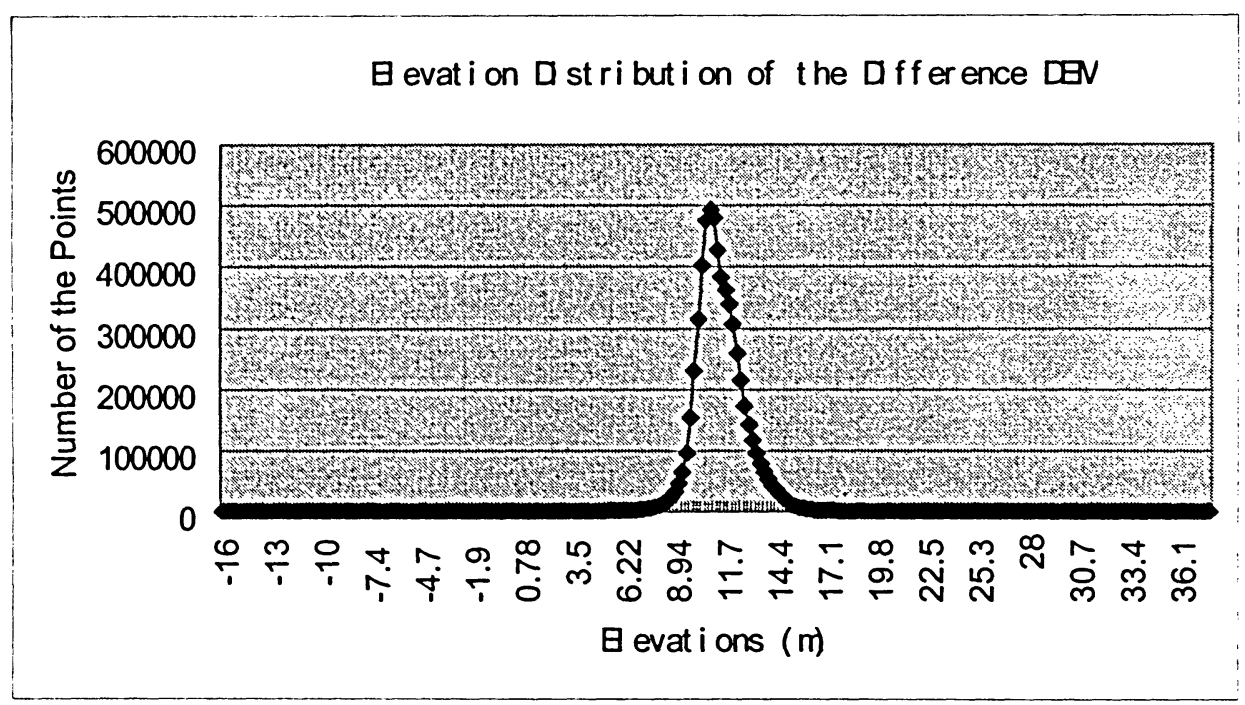

Figure 5-5 Elevation distribution of the difference DEM between the DEM derived using the 3D physical model and the DEM derived using the RFM with vendor-supplied RPCs. 


\subsection{Comparison of Extracted DEMs with "True" Terrain}

To further test the accuracy of the extracted DEMs, the DEM generated using the 3D physical model and the DEM generated using the RFM are then compared to a high accuracy DEM which was manually edited from aerial photographs. The elevation accuracy of such a "ground truth" DEM used in this study is $0.5 \mathrm{~m}$, and it has the same pixel size $(3 \mathrm{~m})$ and projection as the DEMs extracted from IKONOS in-track stereo images. Figure 5-6 shows the DEM used as a "true" terrain for the comparison purpose. Since the DEMs generated in this study are actually DSM, the compare area selected for the comparison is an area with small part of buildings, which will be edited later. Figures 5-7 and 5-8 show the DEM generated using the 3D physical model and the DEM generated using the RFM, respectively. It can be seen that, the DEMs generated in this study contain the elevations of the buildings, which was not shown in the "true" terrain depicted in Figure 5-6. 


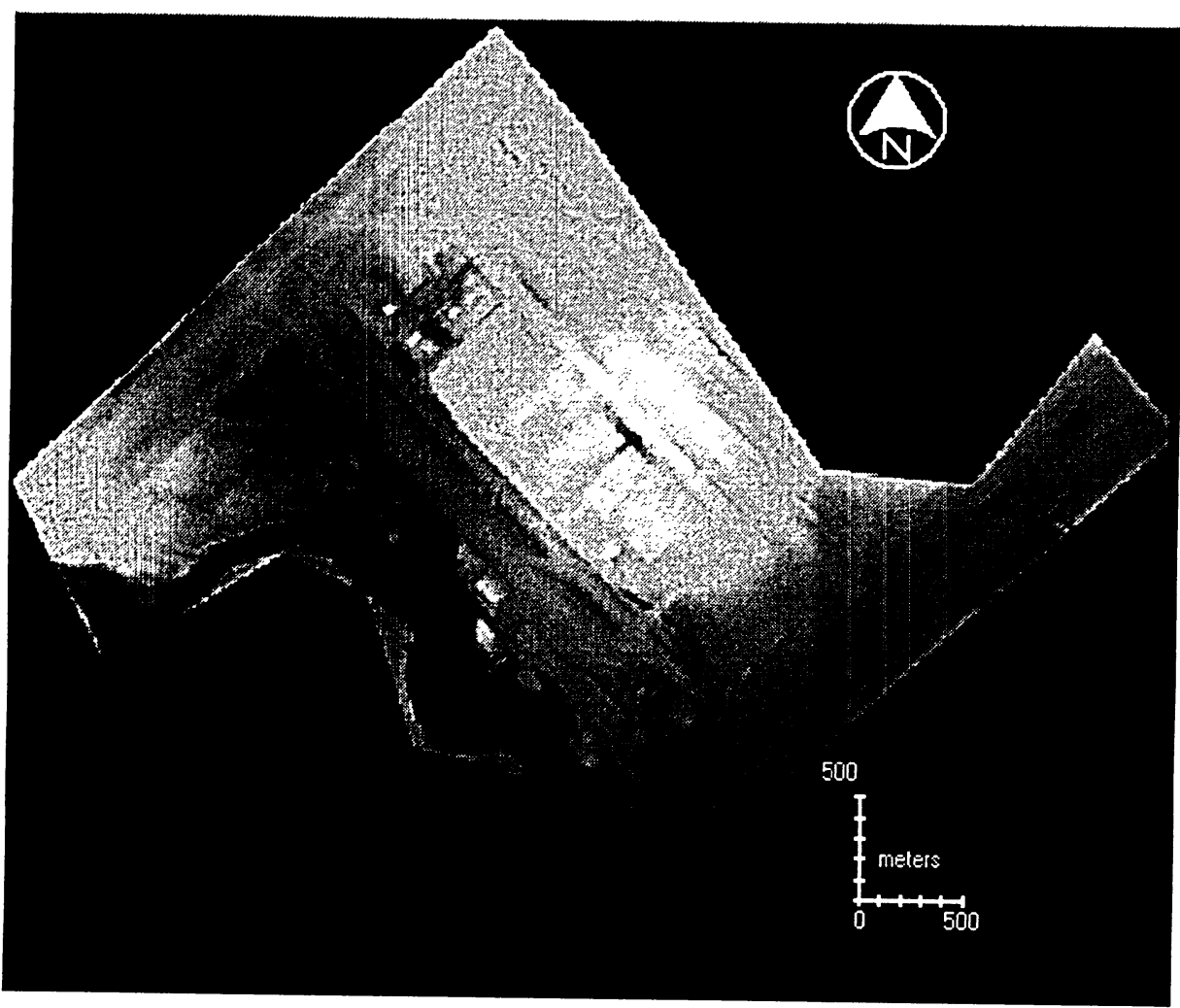

Figure 5-6 "Ground truth" DEM manually edited from aerial photographs.

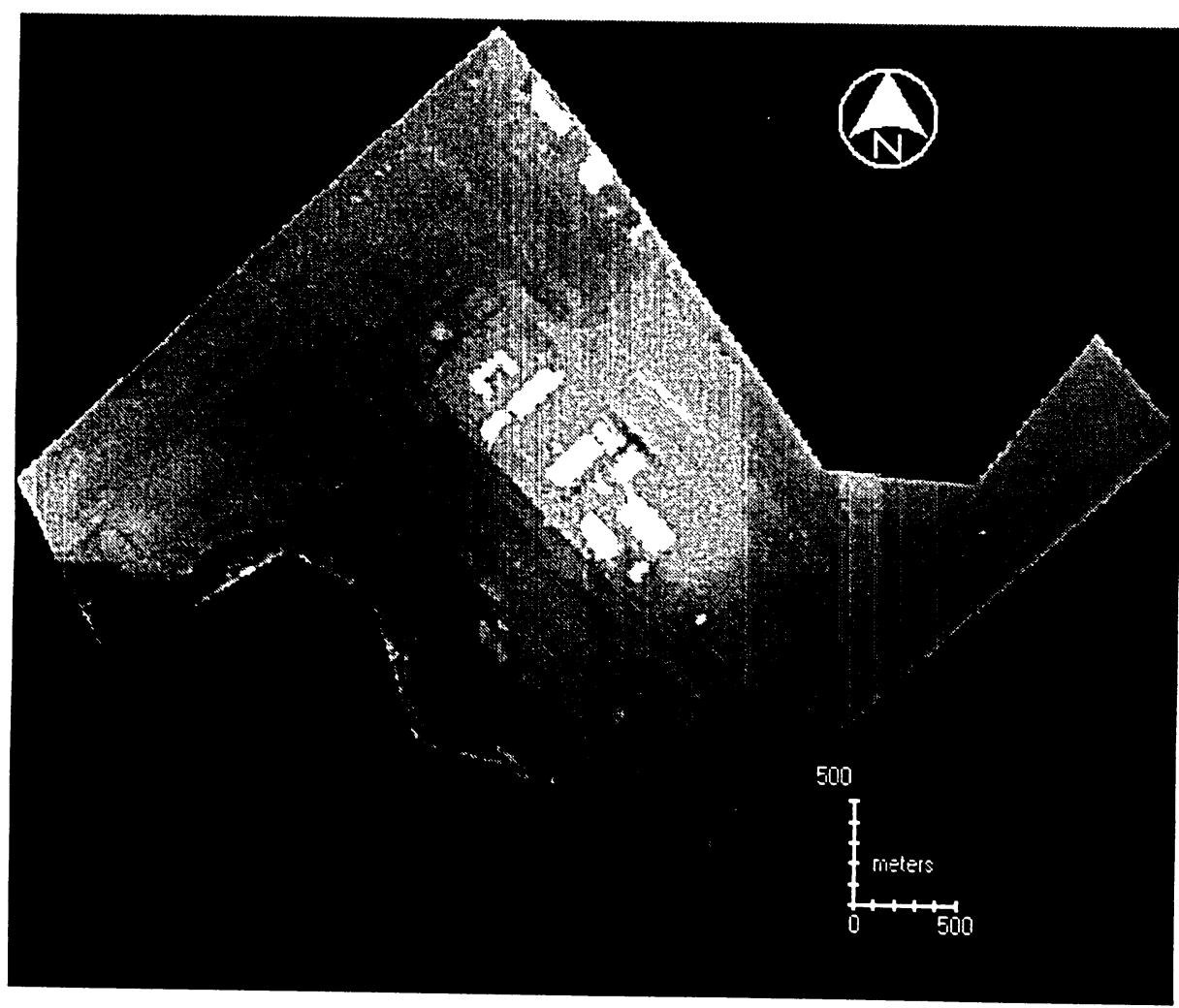

Figure 5-7 DEM generated using the 3D physical model. 


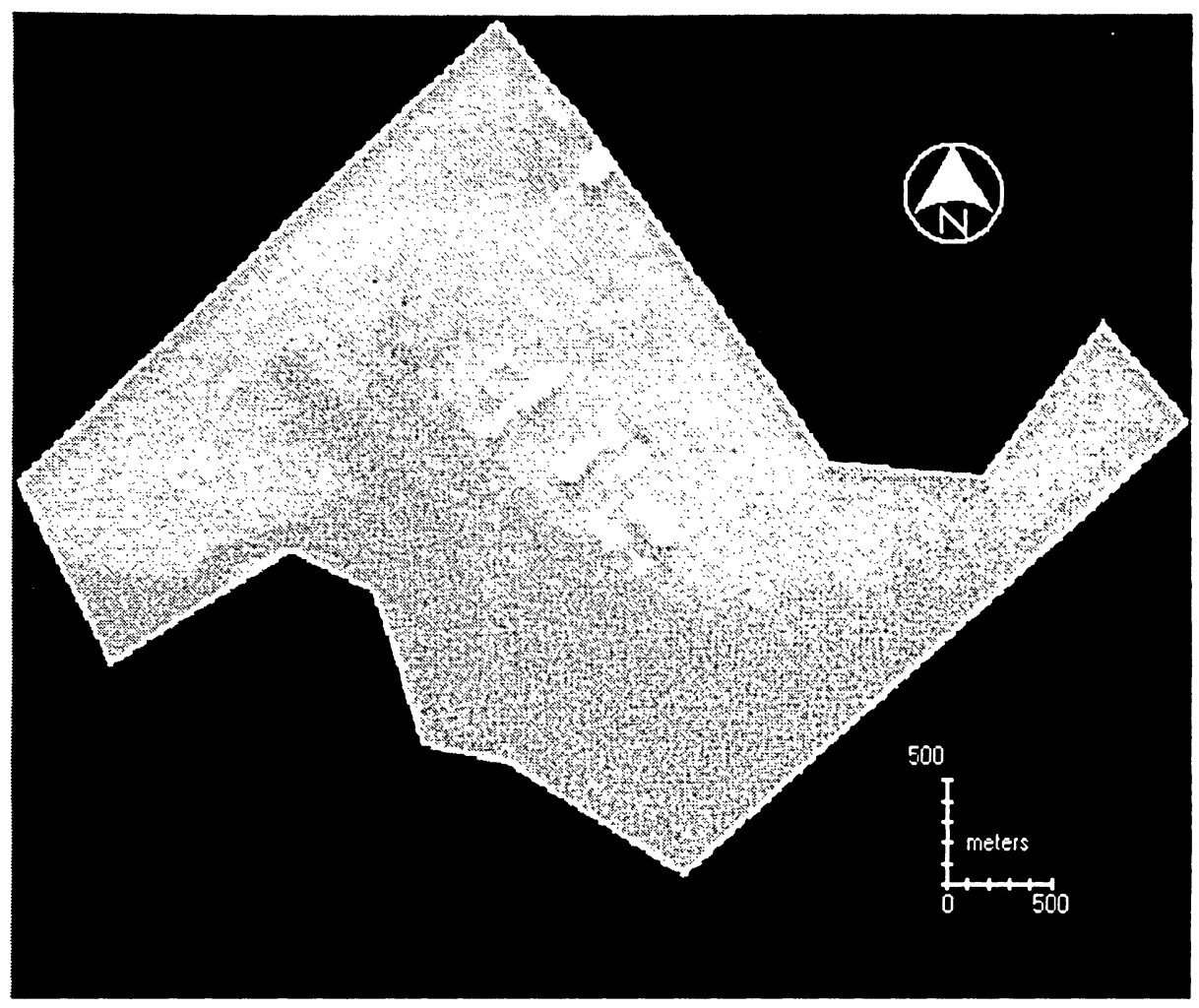

Figure 5-8 DEM generated using the RFM.

To eliminate the influence of the buildings, several masks were set over the buildings to replace the elevations in the building area with the surrounded elevations using a $1^{\text {st }}$ order polynomial function. Figure 5-9 shows the masks over the buildings and also the results of the interpolation. It can be seen that area with buildings does not show any higher elevation values than the surrounding area. 


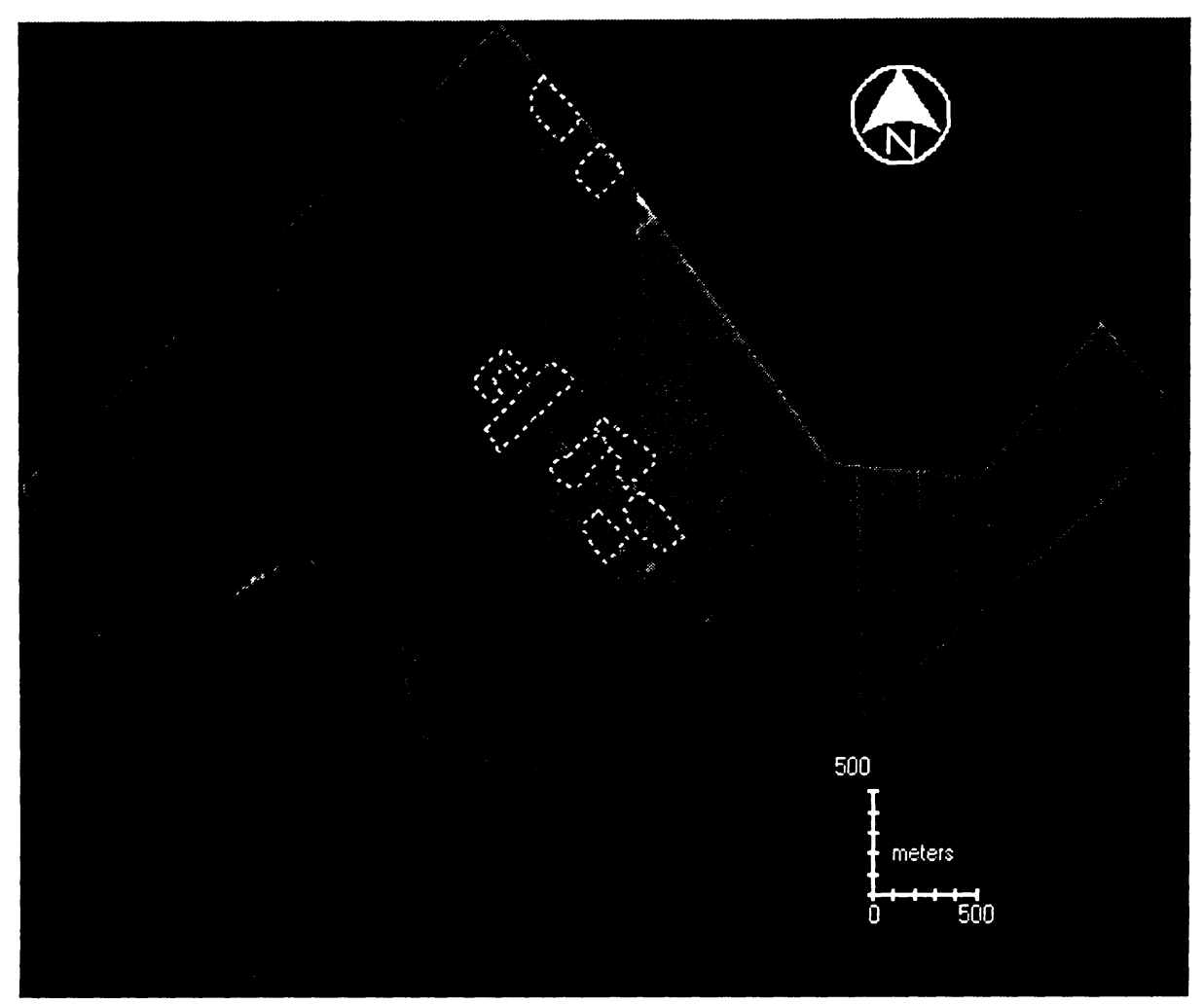

Figure 5-9 The DEM after the interpolation processing of the building area.

After removing all buildings, the two DEMs generated from the 3D physical model and the RFM, respectively, are compared to the "true" terrain. Figures 5-10 and 5-11 show the difference DEMs got from this performance. The elevation of the difference DEMs are also expressed in the level of gray. The darker the area shows the small difference of the two DEMs compared. And Figures 5-12 and 5-13 show the elevation distribution the two sets of difference DEMs. 


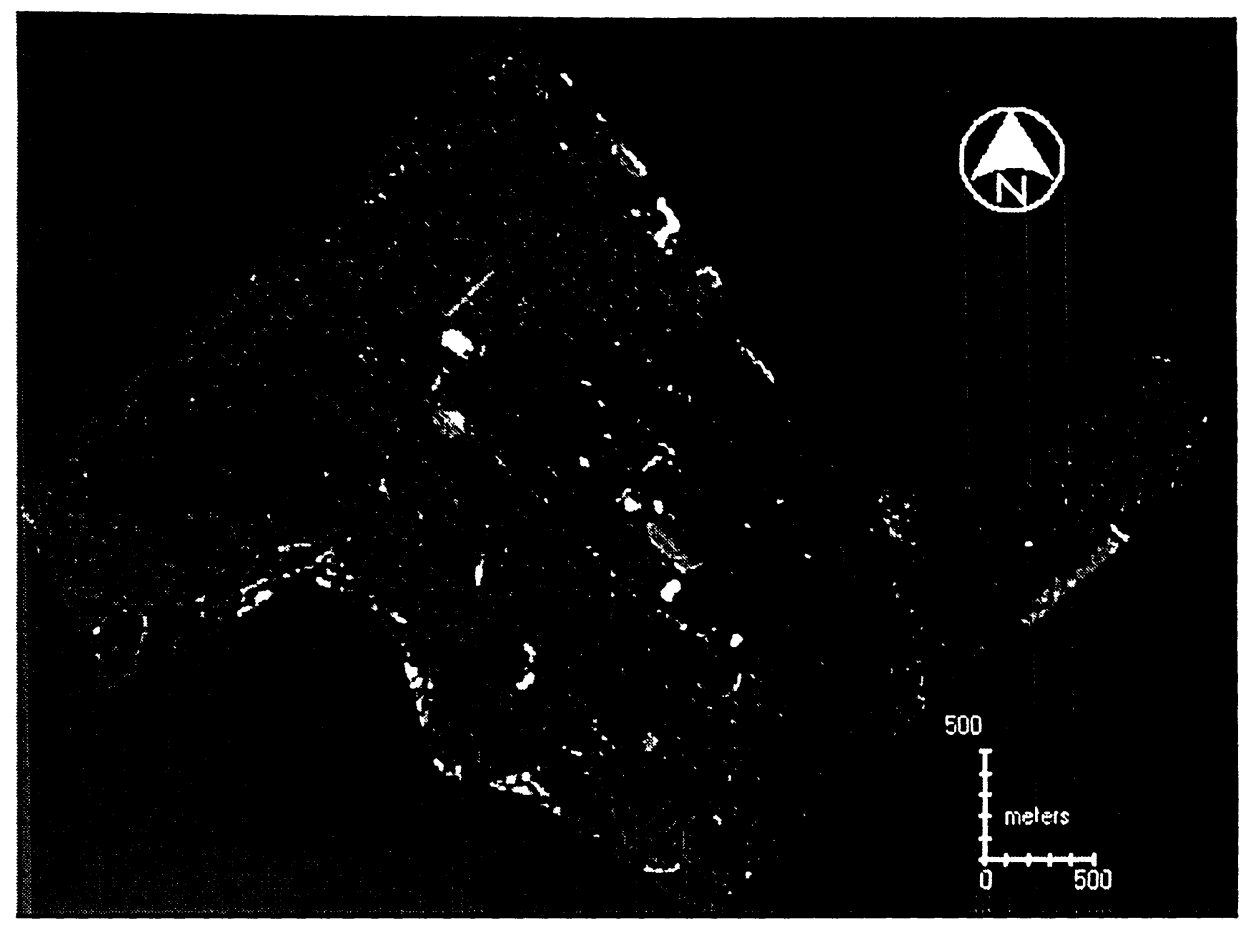

Figure 5-10 Difference DEM between the DEM extracted using the 3D physical model and the "true" terrain.

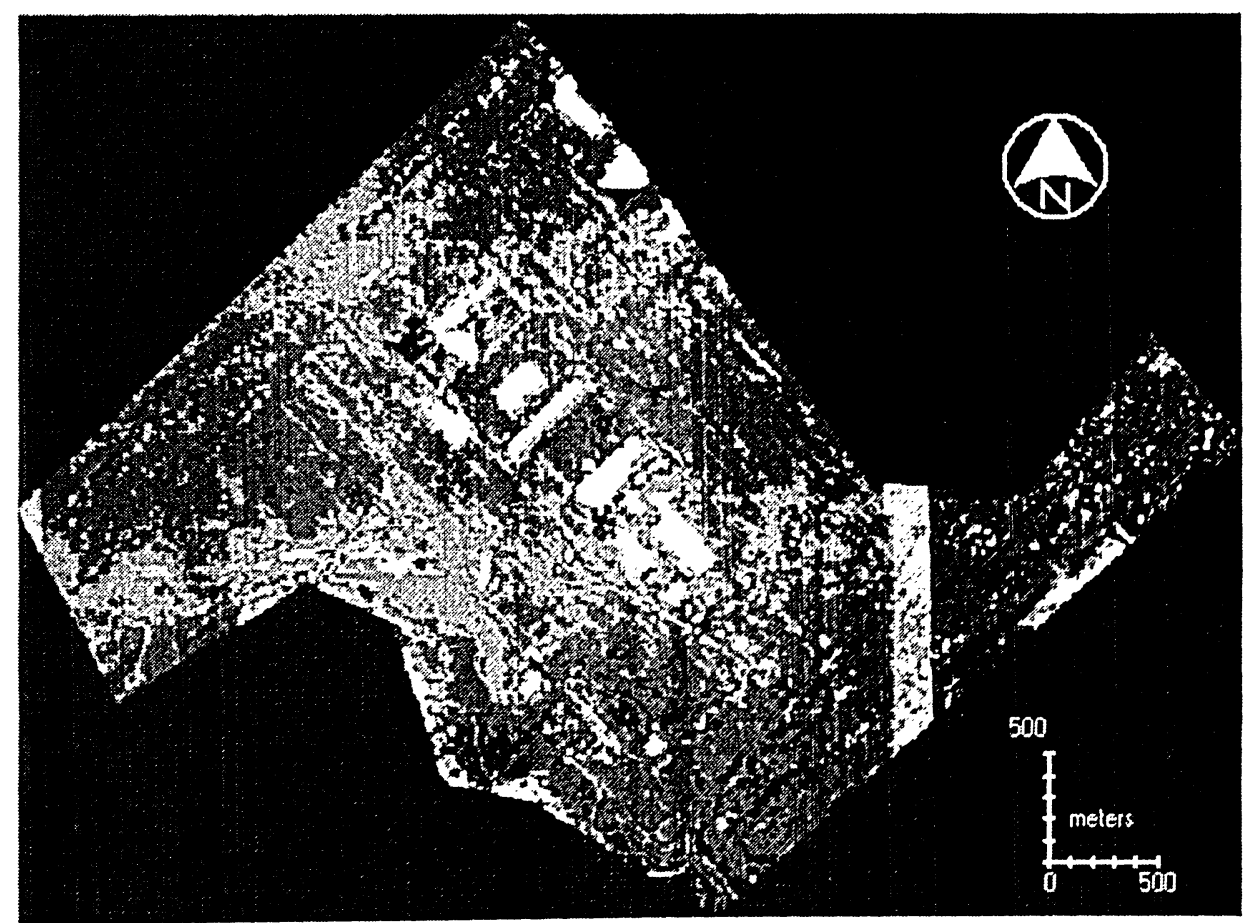

Figure 5-11 Difference DEM between the DEM extracted using the RFM and the

$$
\text { "true" terrain. }
$$




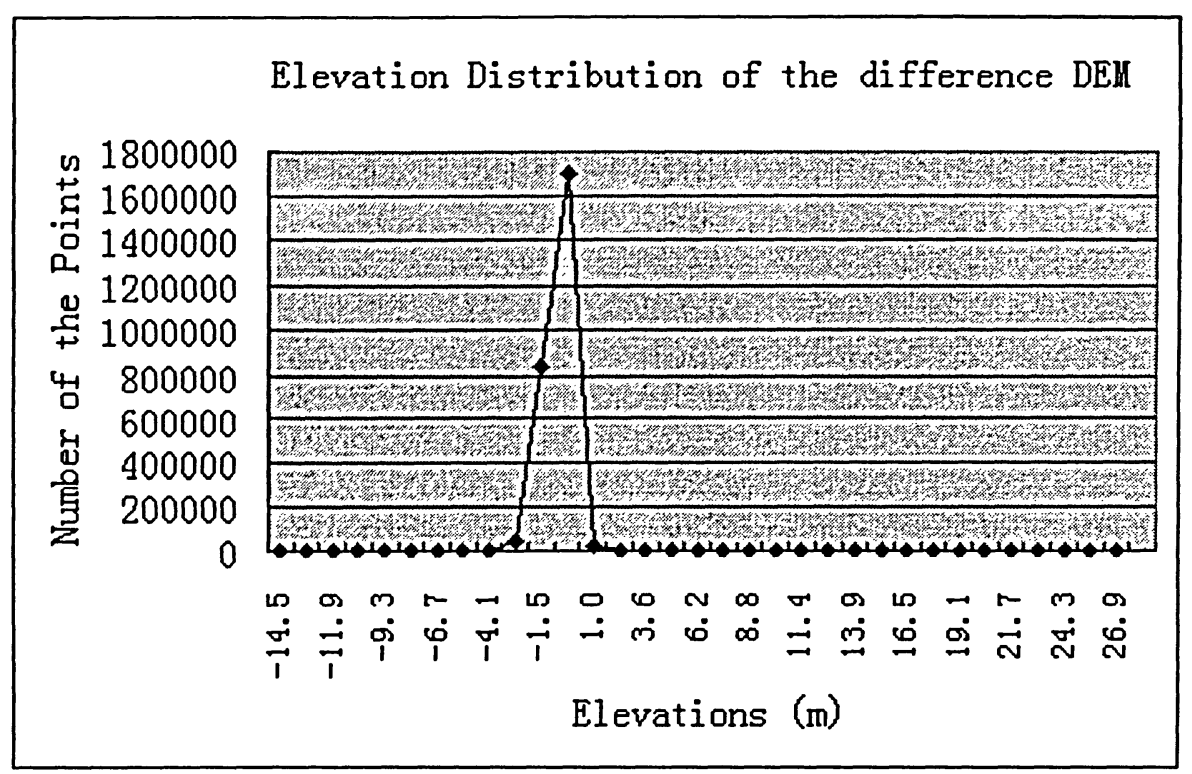

Figure 5-12 Elevation distribution of the difference DEM between the DEM extracted using the 3D physical model and the "true" terrain.

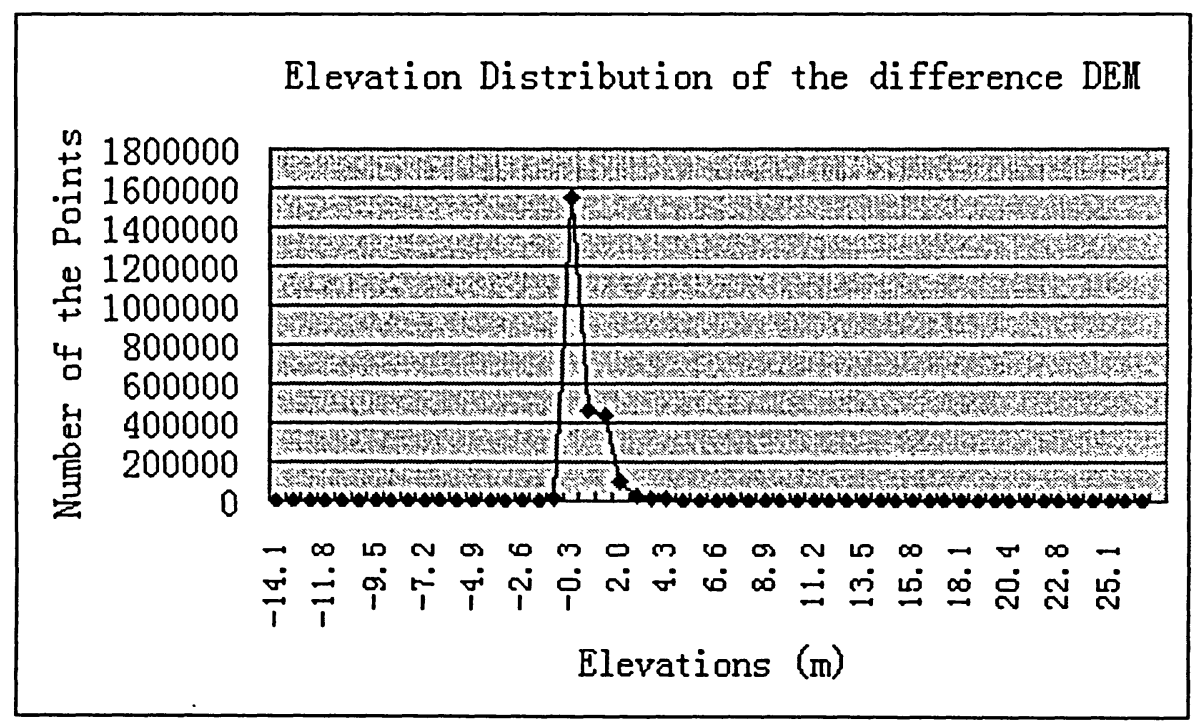

Figure 5-13 Elevation distribution of the difference DEM between the DEM extracted using the RFM and the "true" terrain.

From the above figures, it can be seen that both the DEMs generated from the 3D physical model and the RFM has small difference to the "true" terrain. Most of the 
elevation differences are around $-2 \mathrm{~m}$ to $2 \mathrm{~m}$, which can be seen from Table 5-3. There are totally $2,627,741$ points used for comparison study. At $97.5 \%$ points for the RFM and $97.8 \%$ points for the $3 \mathrm{D}$ physical model of points, elevation differences range from $-2 \mathrm{~m}$ to $2 \mathrm{~m}$, respectively.

Table 5-3 Statistical results of the difference between the generated DEMs and the "true" terrain

\begin{tabular}{|c|c|c|c|c|c|c|}
\hline $\begin{array}{c}\text { Source of the } \\
\text { Difference DEM }\end{array}$ & $\begin{array}{c}\text { Min } \\
(\mathrm{m})\end{array}$ & $\begin{array}{c}\text { Max } \\
(\mathrm{m})\end{array}$ & $\begin{array}{c}\text { Median } \\
(\mathrm{m})\end{array}$ & $\begin{array}{c}\text { Mode } \\
(\mathrm{m})\end{array}$ & $\begin{array}{c}\text { Mean } \\
(\mathrm{m})\end{array}$ & $\begin{array}{c}\text { STDE } \\
(\mathrm{m})\end{array}$ \\
\hline $\begin{array}{c}\text { 3D Physical Model } \\
\text { "minus" "True" } \\
\text { Terrain }\end{array}$ & -14.5 & 24.5 & -0.3 & -0.3 & -0.3 & 1.31 \\
\hline $\begin{array}{c}\text { RFM "minus" } \\
\text { "True" Terrain }\end{array}$ & -14.1 & 26.6 & -0.3 & -0.3 & 0.6 & 1.10 \\
\hline
\end{tabular}

\subsection{Chapter Summary}

The elevation accuracy of the extracted DEMs was evaluated in this chapter. Three methods are used to perform the evaluation. The first is to calculate the elevation errors of ICPs on geocoded DEMs. The DEMs generated using the refined RFM and the 3D physical model have the same level of accuracy on the ICPs, while the DEM generated using the RFM with the vendor-supplied RPC shows lower level of accuracy due to the exist of the systematic errors. The second is to compare the DEM extracted using the RFM with the DEM extracted using the 3D physical model. The difference between the DEM extracted using the refined RFM and the 3D physical model is small, more than $99 \%$ of the differences range from $-4.9 \mathrm{~m}$ to $2.6 \mathrm{~m}$. And the last is to compare the 
DEMs from the 3D physical model and the refined RFM to the "true" terrain. Both of the extracted DEMs show very good accuracy. More than $97 \%$ of the differences range from $-2 \mathrm{~m}$ to $2 \mathrm{~m}$. 


\section{CONCLUSIONS AND RECOMMENDATIONS}

In this study the accuracy potential of IKONOS in-track stereo images for DEM generation was investigated. In this chapter, the major achievements of the approach used to perform the DEMs generation are summarized in Section 6.1. Some limitations are discussed in Section 6.2. Based on the previous discussions, conclusions are drawn in Section 6.3. Finally, recommendations for future research are given in Section 6.4.

\subsection{Summary}

One major drawback to the efficient and appropriate use of IKONOS stereo data is the difficulty for the end users to geometrically process and extract 3D information because the vendor do not provide the rigorous sensor model of IKONOS imagery. This is also the major problem when extracting DEMs from IKONOS stereo images. In this study, IKONOS Reference level stereo images were used for DEM extraction, in which the major tasks performed include GCP collection, 3D reconstruction, automatic stereo image matching, DEM generation, and DEM editing. The satellite-derived DEMs using different mathematical models were then compared, and a quantitative evaluation on elevation accuracy of the final DEM was performed. Two kinds of mathematical models for IKONOS in-track stereo images were used for 3D reconstruction and DEM generation. The main discussion performed lies on the accuracy of the $3 \mathrm{D}$ reconstruction using the RFM and the 3D physical model developed at CCRS. The 
study aims at the investigation on the potential accuracy of the IKONOS stereo images, the metric quality of the IKONOS data, and the examining of practical means for 3D ground point determination from stereo images. Since there is no rigorous mathematical model of IKONOS imagery available for the end users, the traditional collinearity-based sensor orientation models are largely precluded. In order to achieve optimal geopositioning accuracy, the RFM approach, with modified vendor-supplied RPCs, was used to compensate the biases. And another popular mathematical model for IKONOS imagery, the 3D physical model developed at CCRS was also employed in this study. In order to improve upon the specified $25.4 \mathrm{~m} \mathrm{CE90} \mathrm{and} 11.8 \mathrm{~m}$ RMSE position accuracy of the IKONOS Reference level stereo images, it was mandatory that there are some GCPs with good quality available. Whereas a single GCP may be sometimes sufficient to compensate the bias in the vendor-supplied RPCs and yield submetre level accuracy of 3D geopositioning using the RFM, and a minimum of 6 GCPs are required for the 3D reconstruction using the $3 \mathrm{D}$ physical model. 3 GCPs were used in this study to compensate the bias when using the RFM and 12 GCPs were used for the $3 D$ reconstruction when using the $3 \mathrm{D}$ physical model.

\subsection{Limitations}

Although the objective of this study was the investigation on the use of IKONOS in-track stereo images for DEM generation, only the RFM and the 3D physical model were studied and implemented. Some limitations can be summarized as follows. 
First, although only two popularly used non-rigorous models, RFM provided by Space Imaging and 3D physical model developed at CCRS were used in this study for the photogrammetric processing of IKONOS stereo images, acceptable results could also be expected using other non-rigorous models such as direct linear transformation (DLT), self-calibration DLT (SDLT), and 3D affine transformation (Valadan and Sadeghian, 2003).

Second, from the vendor-supplied RPCs, it is apparent that some coefficients in the higher-order polynomial are so small comparing to the lower order polynomial coefficients. They may have little contribution to the $3 \mathrm{D}$ reconstruction results. If some coefficients could be eliminated, the number of GCPs needed to recalculate the vendor-supplied RPCs can be less than the number used in this study.

Third, because the GCPs collected from the existing high-accuracy DEM derived from aerial photographs cover only the central part of the entire IKONOS image frame, the accuracy of only this part of DEM excluding the remaining areas of the final DEM derived from IKONOS in-track stereo images were evaluated.

Last, the study area, located in north of Toronto nearby the Toronto Pearson International Airport, is relatively flat, so the effect of large elevation difference to the accuracy of the $3 \mathrm{D}$ reconstruction using different mathematical models was not investigated in this study. 


\subsection{Conclusions}

Rigorous models are not always available for satellite sensor orientation, especially for images from high resolution satellites such as IKONOS. Unlike the rigorous physical sensor model, non-rigorous models such as RFM, DLT, SDLT and 3D affine transformation need neither the knowledge of the sensor model nor of orbit ephemeris and platform orientation parameters. Applications of these models to stereo images acquired by IKONOS satellite indicate that relatively accurate geopositioning can be obtained through provision of ground control information.

This thesis has presented the method for the extraction of DEMs from IKONOS in-track stereo images using the RFM and the 3D physical model. Its application to the real case studies shows the accuracy and usefulness of these methods. Several findings and conclusions are summarized below:

- The experiments with the IKONOS stereo images using the derived equations of the RFM 3D reconstruction verified that the RFM can approximate the rigorous sensor models very accurately and can be used for the DEMs extraction.

- The accuracy of the 3D reconstruction performed using the vendor-supplied RPCs was significantly affected by a systematic error of up to $12 \mathrm{~m}$ in the $\mathrm{Z}$ direction. With a few number of GCPs, this systematic bias can be compensated to submetre level of RMSE on the ICPs.

- Three ways proved robust for improving the geopositioning accuracy of IKONOS 
stereo images with good quality GCPs by refining the vendor-supplied RPCs, refining the RFM derived ground coordinates, or by recalculating the vendor-supplied RPCs. The accuracy of IKONOS Reference level stereo products can then be improved to the Precision plus level products which has the $0.9 \mathrm{~m}$ RMSE of positional accuracy. Therefore, the RFM provides a cost-effective method for high accuracy DEM generation.

- From the three methods mentioned above, the method for bias compensation by refining the vendor-supplied RPCs is recommended since it is easy to perform and fewer GCPs are required.

The 3D physical model available in the PCI Geomatica OrthoEngine environment can be used for the accurate processing of IKONOS stereo images, and extracting 3D information. By providing approximate 12 to 15 high-accuracy GCPs, the submetre level accuracy of the 3D reconstruction could be achieved.

It can be concluded that IKONOS stereo imagery is clearly of very high metric quality, and it is a relatively straightforward matter to achieve high $3 \mathrm{D}$ geopositioning for the DEM generation with the Reference level of IKONOS stereo product.

\subsection{Recommendations for Future Research}

Based on the proposed methodology and the experimental results, some 
recommendations can be summarized in order to extend the research in the future.

- When using the refined RFM, the contribution of the individual vendor-supplied RPCs may vary to the 3D sensor model calculation. Those coefficients with smaller contribution which may lead to unstable recalculation of RPCs should be removed.

- Large errors may occur in areas shadowed due to hills, buildings, and trees (no leaves on deciduous trees in winter-acquired stereo images). Because the surface heights are included in terrain elevation, the elevation errors should be considered as a function of the land cover (bare surfaces, urban areas, deciduous, conifer, mixed, and spare forests). It is suggested that DEM accuracy may be evaluated by classifying the study area into different terrain types (e.g., hilly topography with different land cover classes).

- High-resolution satellite images increase the need for higher accuracy of data modeling. Other non-rigorous models such as DLT, SDLT and 3D affine transformation can be used to extract DEMs from IKONOS in-track stereo images to see if a better DEM can be expected. In order to accurately model the imaging geometry of high-resolution flexible pointing images such as IKONOS, a satellite orbital parameter model is useful. 


\section{BIBLIOGRAPHY}

Ager, T. P., 2003. Evaluation of the geometric accuracy of Ikonos imagery, Proceedings of SPIE 2003 AeroSense Conference, 21-25 April, Orlando, Florida.

Al-Rousan, N., P. Cheng, G. Petrie, T. Toutin, and M.J. Valadan Zoej, 1997. Automated DEM extraction and orthoimage generation from SPOT level 1B imagery, Photogrammetric Engineering \& Remote Sensing, 63 (8): 965-974.

Ayeni, O. O., 1982. Optimum sampling for digital terrain models: A trend towards automation, Photogrammetric Engineering \& Remote Sensing, 48(11): 1687 1694.

Baltsavias, E. P., and D. Stallmann,1992. Metric information extraction from SPOT images and the role of polynomial mapping functions, International Archives of Photogrammetry and Remote Sensing, 29(B4): 358-364.

Baltsavias, E., M. Pateaki and L. Zhang, 2001. Radiometric and geometric evaluation of IKONOS geo images and their use for 3D building modeling, Joint ISPRS Workingshop on High Resolution mapping from Space 2001, 19-21 September, Hannover, Germany.

Cheng, P. and T. Toutin, 1997. Urban planning using data fusion of satellite and aerial photo images, Proceedings of IGARSS'97, 2(2): 839-841.

Cheng, P., T. Toutin, Y. Zhang and M. Wood, 2003. Quickbird: geomatic correction, path and block processing and data fusion. Earth Observation Magazine, 12(3): 24-30.

Cheng, P., and T. Toutin, 2000. Orthorectification of IKONOS data using rational function, Proceedings of ASPRS Annual Convention, 22-26 May, Washington, DC.

Davis, C. H., and X. Wang, 2001. High resolution DEMs for urban applications from NAPP photography, Photogrammetric Engineering and Remote Sensing, 67(5): 585-592.

Davis, C. H., and W. Wang, 2001. Planimetric accuracy of IKONOS 1-m panchromatic image products, Proceedings of the ASPRS Annual Conference, 23- 27 April, St. Louis, CD-ROM, 14 p.

Dhond, U. R., and J. K. Aggarwal, 1989. Structure form stereo-a review, IEEE Transactions on System, Man and Cybernetics, 19(6): 1489-1510. 
Dial, G., 2000. IKONOS satellite mapping accuracy, Proceedings of the ASPRS Annual Conference, 22-26 May, Washington, DC, CD-ROM, 8 p.

Dial, G., 2001. IKONOS overview, Proceedings of the High-Spatial Resolution Commercial Imagery Workshop, March 19-22, Washington DC.

Dial, G., and J. Grodecki, 2002. Block adjustment with rational polynomial camera models, Proceedings of the ACSM-ASPRS Annual Conference/22 $2^{\text {nd }} F I G$ International Congress, April 19-26, Washington D.C., CD-ROM.

Dial, G. and J. Grodecki, 2002. IKONOS accuracy without ground control, International Archives of Photogrammetry, Remote Sensing and Spatial Information Sciences, 34(1).

Dial, G. and J. Grodecki, 2004. Satellite image block adjustment simulations with physical and RPC camera models, Proceedings of the ASPRS Annual Conference, 23-28 May, Denver, Colorado.

Dial, G. and J. Grodecki, 2004. RPC replacement camera models. International Archives of Photogrammetry, Remote Sensing and Spatial Information Sciences, 34(B1).

Digitalglode, 2001. QuickBird Imagery Products Product Guide, URL: http://www.digitalglobe.com/downloads/QuickBird Imagery Products - Product Guide.pdf. (Last date accessed: 8 April 2005).

Di, K., R. Ma, and R. Li, 2001. Deriving 3D shorelines form high resolution Ikonos satellite images with rational functions, Proceedings of ASPRS Annual Convention, 25-27 April, St. Louis, Missouri.

Di, K., R. Ma, and R. Li, 2003. Rational functions and potential for rigorous sensor model recovery. Photogrammetric Engineering \& Remote Sensing, 69(1): 33-41.

Di, K., R. Ma, and R. Li, 2003. Geometric processing of IKONOS stereo imagery for coastal mapping applications. Photogrammetric Engineering \& Remote Sensing, 69(8): 873-979.

Dowman, I., and C. V. Tao, 2002. An update on the use of rational functions for photogrammetric restitution, Highligths ISPRS, 7(3): 22-29.

Dowmann, I., and J. Dolloff, 2000, An evaluation of rational function for photogrammetric restitution, International Archives of Photogrammetry and Remote Sensing, 33 (B3): 254- 266. 
Elachi, C., 1998. Spaceborne Radar Remote Sensing: Applications and Techniques, IEEE Press, New York.

El-Manadili, Y., and K, Novak, 1996. Precison rectification of SPOT imagery using the direct linear transformation model, Photogrammetic Engineering \& Remote Sensing, 62(1): 67-72.

ERDAS, 2001, IKONOS Sensor Model Support Tour Guide. URL: http://www.unc.edu/atn/gis/arcview/pc_manuals_33/IKONOS.pdf. (Last date accessed: 8 April 2005).

Fraser, C., and J. Shao, 1996. Exterior orientation determination of MOMS-02 three-line imagery: Experiences with the Australian testified area, International Archives of Photogrammetry and Remote Sensing, 31(B3): 207-214.

Fraser, C. S., 2000. High-resolution satellite imagery: A review of metric aspects, International Archives of Photogrammetry and Remote Sensing, 33(PartB7): 452-459.

Fraser, C. S., H. B. Hanley and T. Yamakawa, 2001. Sub-metre geo-positioning with IKONOS Geo imagery. Proceedings of Joint ISPRS Workshop on High Resolution Mapping from Space, 19-21 September, Hannove, Germany, CD-ROM, pp. 61-68.

Fraser, C. S., H. B. Hanley, and T. Yamakawa, 2002, Three-dimensional geopositioning accuracy of IKONOS imagery, Photogrammetric Record, 17(99): 465-479.

Fraser, C. S., E. Baltsavias, and A. Gruen, 2002. Processing of IKONOS imagery for sub-metre3D positioning and building extraction, ISPRS Journal of Photogrammetry and Remote Sensing, 56(3): 177-194.

Fraser, C.S., H. B. Hanley. and T. Yamakawa, 2002. High precision geopositioning from Ikonos satellite imagery. Proceedings ASPRS Annual Meeting, 22-26 May, Washington, DC, CD-ROM, 9 p.

Fraser, C. S., and H. B. Hanley, 2003. Bias compensation in rational functions for IKONOS satellite imagery. Photogrammetric Engineering \& Remote Sensing, 69(1): 53-58.

Fritsch, D. and D. Stallmann, 2000. Rigorous photogrammetric processing of high resolution satellite imagery, International Archives of Photogrammetry and Remote Sensing, 33(B1).

Fritsch, D., and D. Tallmann, 2000. Rigorous photogrammetric prociessing of 
high-resolution satellite imagery, International Archives of Photogrammetry and Remote Sensing, 33(B1):313-321.

Fritz, L. W., 1996. The era of commercial Earth observation satellite, Photogrammetric Engineering \& Remote Sensing, 62 (1): 39-45.

Ganas, A., E. Lagios, and N. Tzannetos, 2002. An investigation into the spatial accuracy of the IKONOS 2 orthoimagery within an urban environment. International Journal of Remote Sensing, 23(17):3513-3519.

Gerlach, F., 2000. Characteristics of Space Imaging's one-meter resolution satellite imagery products International Archives of Photogrammetry and Remote Sensing, 33(B1): 128-135.

Gong, J., Z. Li, Q. Zhu, H. Sui, and Y. Zhou, 2000. Effects of various factors on the accuracy of DEMs: an intensive experimental investigation. Photogrammetric Engineering \& Remote Sensing, 66(9): 1113-1117.

Gonzalez, R. C. and R. E. Woods, 2001. Digital Image Processing, Prentice-Hall,. Upper Saddle River, New Jersey, 793p.

Greenfeld, J., 2001. Evaluating the accuracy of digital orthophoto quadrangles (DOQ) in the context of parcel-based GIS. Photogrammetric Engineering \& Remote Sensing, 67(2): 199-205.

Grodecki, J., and G. Dial, 2001. IKONOS Geometric Accuracy, Proceedings of Joint ISPRS Workshop on High Resolution Mapping from Space, 19-21 September, Hannove, Germany, CD-ROM, pp. 77-86.

Grodecki, J., and G. Dial, 2003. Block adjustment of high-resolution satellite images described by rational functions. Photogrammetric Engineering \& Remote Sensing, 69(1):59-69.

Grodecki, J., 2001. IKONOS stereo feature extraction - RPC approach. Proceedings of the ASPRS Annual Conference, 23-27 April, St. Louis, CD-ROM, 7 p.

Grodecki, J. and G. Dial, 2002. IKONOS geometric accuracy validation. International Archives of Photogrammetry, Remote Sensing and Spatial Information Sciences, 34(1): 10- 15.

Grodecki, J., G. Dial and J. Lutes, 2003. Error propagation in block adjustment of high-resolution satellite images, Proceedings of the 2003 ASPRS Annual Conference, 5-9 May, Anchorage, Alaska. 
Grodecki, J. and G. Dial, 2003.Block Adjustment of high-resolution satellite images described by rational polynomials, Photogrammetric Enginecring \& Remote Sensing, 69(1): 59-68.

Gruen, A., 2000. Potential and limitations of high-resolution satellite imagery. Keynote Address, Proceedings of the 21st Asian Conference on Remote Sensing, $4-8$ December, Taipei, Taiwan, $13 \mathrm{p}$.

Gupta, R., and R. Hartley, Linear pushbroom cameras, IEEE Transactions on PAMI 19 (9): 963-975.

Gülch, E., 1991. Results of test on image matching of ISPRS WG III/4, ISPRS Journal of Photogrammetry and Remote Sensing, 46(1): 1-8.

Hanley, H.B., and C.S. Fraser, 2001. Geopositioning accuracy of IKONOS imagery: Indications from two dimensional transformations, Photogrammetric Record, 17(98):317-329.

Hipple, J., and D. Daugherty, 2000. Urban validation site for testing impervious surface models derived from remotely sensed imagery. Proceedings of International Geoscience and Remote Sensing Symposium, 24-28 July, Honolulu, Hawaii, 7: 2885-2889.

Hu, Y., and C.V. Tao, 2002. Updating solutions of the rational function model using additional control information. Photogrammetric Engineering \& Remote Sensing, 68 (7): 715-723.

Hu, Y., and C.V. Tao, 2001. 3-D reconstruction algorithms with the rational function model and their applications for Ikonos stereo imagery. Proceedings of the Joint ISPRS Workshop on High Resolution Mapping from Space 2001, 19- 21 September, Hannover, Germany, CD ROM,12 p.

Kim, T., 2000. A study on the epipolarity of linear pushbroom images, Photogrammetric Engineering \& Remote Sensing, 66 (8):961-966.

Kornus, W., and M. Lehner, 1998. Photogrammetric point determination and DEM generation using MOMS-2P/PRIRODA three-line imagery, International Archives of Photogrammetry and Remote Rensing, 32(B4): 321-328.

Kratky, W., 1989. On-line aspects of stereophotogrammetric processing of SPOT images, Photogrammetric Engineering \& Remote Sensing, 55(3): 311-316.

Krupnik, A., 2000. Accuracy assessment of automatically derived digital elevation models from SPOT images, Photogrammetric Engineering \& Remote Sensing, 
66(8): 1017-1023.

Leberl, F. W., 1990. Radargrammetric Image Processing, Artech House, Norwood, USA $595 \mathrm{p}$.

Li, J, 2004. Satellite-derived DEMs: comparison of accuracy, cost and time with airphotos, Proposal submitted to the Ontario Ministry of Transportation for the Highway Infrastructure Innovation Funding Program, 9p.

Li, R., 1998. Potential of high-resolution satellite imagery for national mapping products, Photogrammetric Engineering \& Remote Sensing, 64 (12): 1165-1170.

Li, R., G. Zhou, S. Yang, G. Tuell, N.J. Schmidt and C. Fowler 2000. A Study of the Potential Attainable Geometric Accuracy of IKONOS Satellite Imagery. International Archives of Photogrammetry and Remote Sensing, 33(B4/2): 587-595.

Lue, Y. and K. Nova, 1991. Recursive grid- dynamic window matching for automatic DEM generation, GIS/LIS ACSM-ASPRS Fall Convention, 1991, PA254-A260.

Lutes, J., 2004. Accuracy analysis of rational polynomial coefficients for IKONOS imagery. Proceedings of ASPRS Annual Conference, May 2004, Denver, Colorado.

Lutes, J. and J. Grodecki, 2004. Error propagation in IKONOS mapping blocks. Photogrammetric Engineering and Remote Sensing, 70 (8): 947-955.

Madani, M., 1999. Real-time Sensor-Independent Positioning by Rational Functions, Proceedings of ISPRS Workshop on Direct Versus Indirect Methods of Sensor Orientation, Barcelona, Spain, November 25-26, (Barcelona, Spain:ISPRS) pp. 64-75.

Madani, E. M., J. S. Bethel, and J.D. McGlone, 2001. Introduction to Modern Photogranmery, John Wiley \& Sons, New York, NY, 479 p.

Maune, D. F., 2001. Digital Elevation Model Technologies and Applications: The DEM Users Manual, American Society for Photogrammetry and Remote Sensing, Bethesda, Maryland, $539 \mathrm{p}$.

McGlone, C., 1996. Sensor Modeling in image registration, In Digital Photogrammetry: an addendum, Edited by C.W. Greve, pp. 115-123.

Mcintosh, J. H. and K. M. Mutch, 1988. Matching straight lines, Computer Vision Graph Image Processing 43, pp 368-408. 
Medioni, G, and R. Nevatia, 1984. Matching image using linear features, IEEE Transactions Pattern and Mach Intell, Vol 6, pp 675-785.

Medinoi, G. and R. Nevatia, 1985. Segment based stereo matching, Computer Vision Image Processing, 31, pp. 2-18.

NIMA, 2000. The Compendium of Controlled Extensions for the National Imagery Transmission Format (NITF). URL: http://www.Ismc.nima.mil/ntb/superceded /STDI-0002_v2.1.pdf. (Last reach, 08 April 2005).

Okamoto, A., T. Ono, S. Akamatsu, C. S. Fraser, S. Hattori, and H. Hasegawa, 1999. Geometric characteristics of alternative triangulation models for satellite imagery, Prodeedings, ASPRS Annual Conference, 17-21 May, Portland, Oregon.

OGC, 1999. The Open GISTM Abstract Specifications: The Earth Imagery Case, Vol. 7, URL: www.opengis.org/techno/specs/htm/ (Last reach, 08 April 2005).

Otto, G., and T. Chau, 1989. A region growing algorithm for matching of terrain image, Image and Vision Computing, 7: 83-94.

Paderes, F. C., Mikhail, and J. A. Fagerman, 1989. Batch and on-line evaluation of stereo SPOT imagery, Proceedings of ASPRS-ACSM Convention, Baltimore, MD, (Bethedsa, MD: ASPRS) Vol. 3, pp. 31-40.

Papapanagiotu, E. G., and J. N. Hatzopoulos, 2000. Automatic extraction of 3D model coordinates using digital stereo images, International Archives of Photogrammetry and Remote Sensing, 33(Part B4/2): 805-812.

Parker, J., 1997. The advantages of In-track stereo acquisition from high-resolution earth resources satellite, Proceedings of ACSM/ASPRS Annual Convention \& Exhibition, 4-6 April, Seattle, Washington, 1:276-282.

PCI Geomatics, 2001. OrthoEngine, Reference Manual, Version 7.0.

PCI Geomatics Web Site, 2001. URL: http://www.pcigeomatics.com/. (Last date accessed: 08 April 2005).

Pollard, S., J. Mayhew, and J. Frisby, 1985. A stereo correspondence algorithm using a disparity gradient limit, Perception, 14: 449-470.

Ridley, H. M., P. M. Atkinson, Pl. Aplin, J.P. Muller, and I. Dowman, 1997. Evaluating the potential of the forthcoming commercial U. S. high-resolution satellite sensor imagery at the Ordnace Survey, Photogrammetric Engineering \& Remote Sensing, 63(8): 997-1005. 
Sadeghian, S., and M. R. Delavar, 2003. An investigation of geometric correction and uncertainty assessment of high resolution images, Proceedings of the $2^{\text {nd }}$ International Symposium on Spatial Data Quality, Hong Kong, pp. 89-99.

Savopol, F., and C. Armenakis, 1998. Modelling of the IRS-1C satellite Pan imagery using the DLT approach, International Archives of Photogrammetry and Remote Sensing, 32(4): 511-514.

Schenk, T., 1999. Digital Photogrammetry, TerraScience, Bailor, Laurelville, 428 p.

Shi, W. and A. Shaker, 2003. Analysis of terrain elevation effects on IKONOS imagery rectification accuracy by using non-rigorous models, Photogrammetric Engineering \& Remote Sensing, 69(12):1359-1366.

Space Imaging, 2001. Image products, URL: http://www.spaceimaging.com/products/ imagery.htm (last date accessed: 8 April 2005).

Tateishi, R., and A. Akutsu, 1992. Relative DEM production from SPOT data without GCP, International Journal of Remote Sensing, 139(14): 2517-2530.

Tao, C. V., Y. Hu, and W. Jiang, Photogrammetric exploitation of IKONOS imagery for mapping applications, International Journal of Remote Sensing, 25(14): 2833-2853.

Tao C. V., and Y. Hu, 2001. A comprehensive study of the rational function model for photogrammetric processing. Photogrammetric Engineering \& Remote Sensing, 67(12):1347-1357.

Tao, C. V., and Y. Hu, 2001. Use of the rational function model for image rectification. Canadian Journal of Remote Sensing, 27(6):593-602.

Tao, C. V., and Y. Hu, 2002. 3-D reconstruction methods based on the rational function model. Photogrammetric Engineering \& Remote Sensing, 68(7):705-711.

Tao, C. V. and Y. Hu, 2002. Photogrammetric exploitation of Ikonos imagery using the rational function model. Proceedings of 2002 ACSM-ASPRS Annual Conference, April 22-26, Washington, DC, CD-ROM, 12 p.

Tao, C. V., and Y. Hu, 2000. Rational function model and applications for photgrammetric processing, URL: http://geomaci.geomatics.ucalgary.ca/project/ rationalmapper/rationalmapper.htm. (Last date accessed: 8 April 2005).

Toutin, T., 1995. Multi-source data integration with an integrated and unified geometric modeling, EARSel Journal of Advances in Remote Sensing, 4(2): 118-129. 
Toutin, T., and P. Cheng, 2001. DEM with stereo Ikonos: a reality, Earth Observation Magazine, 10(7):13-17.

Toutin, T. and P. Cheng, 2000. Demystificaiton of IKONOS, Earth Observation Magazine, 9(7):17-21.

Toutin T., 2001. DEM generation from new VIR sensors: IKONOS, ASTER and Landsat-7, IGARSS'2001, July 9-13, Sydney, Australia.

Toutin, T., 2001. Geometric processing of Ikonos Geo images with DEM. Proceedings of the Joint ISPRS Workshop on High Resolution Mapping from Space 2001, 1921 September, Hannover, Germany, CD-ROM, 9 p.

Toutin, T., 2002. Block bundle adjustment of Ikonos in-track images, International Journal of Remote Sensing, 24(4): 51-857.

Toutin, T., R. Chénier, and Y. Carbonneau, 2002. 3D Models for high resolution images: examples with QuickBird, IKONOS and EROS, International Archives of Photogrammetry and Remote Sensing, 34(4).

Toutin, T. and P. Cheng, 2002. QuickBird: a milestone for high resolution mapping, Earth Observation. Magazine., 11 (4): 14-18, 2002.

Toutin, T., 2003. Error tracking in IKONOS geometric processing using a 3D parametric modeling, Photogrammetric Engineering \& Remote Sensing, 69(1): 43-51.

Toutin, T., 2004. Geometric processing of remote sensing images: models, algorithms and methods, International Journal of Remote Sensing, 25(10): 1893-1924.

Toutin, T., 2004. DTM generation from Ikonos in-track stereo images using a 3D physical model. Photogrammetric Engineering \& Remote Sensing, 70 (6): 695702.

Toutin, T., 2004. Comparison of stereo-extracted DTM from different high-resolution sensors: SPOT-5, EROS-A, IKONOS-II, and QuickBird, IEEE Transactions on Geoscience and Remote Sensing, 42(10):2121-2129.

Valadan, M. J. And S. Sadeghian, 2003. Rigorous and non-rigorous photogrammetric processing of Ikonos Geo image, Proceedings of the Joint Workshop on High Resolution Mapping from Space 2003, October 6-8, Hannover, Germany, CD-ROM, 6p. 
Wang, Y., 1999. Automated triangulation of linear scanner imagery. Proceedings of the Joint ISPRS Workshop on Sensors and Mapping from Space, Hannover, 27-30 September, Hannover, Germany, CD-ROM, 5 p.

Wang, Z., 1990. Principle of Photogrammetry: with Remote Sensing, Press of Wuhan Technical University of Surveying and Mapping and Publishing House of Surveying and Mapping, Wuhan, China, 575p.

Waston, D., 1992. Contouring: A Guide to the Analysis and Display of Spatial Data, Pergamon Press, England.

Whiteside, A., 1997. Recommended Standard Image Geometry Models, OpenGIS Web Site, URL: http://www.opengis.org/ipt/9702tf/UniversalImage/TaskForce.ppt (Last date accessed: 8 April 2005).

Wolf, P. R. and B. A. Dewitt, 2000. Elements of Photogrammetry: with Application in GIS, 3rd edition, McGraw-Hill, New York, 608p.

Yang, X., 2000. Accuracy of rational function approximation in photogrammetry. Proc. ASPRS Annual Conference, 22-26 May, Washington D.C., CD-ROM ,10 p.

Yang, Y., 2001. Piece-wise linear rational function approximation in digital photogrammetry. Proceedings of the ASPRS Annual Conference, 23-27 April, St Louis, CD-ROM, 14 p.

Zhang, Z., J. Zhang, M. Liao, and L. Zhang, 2000. Automatic registration of multi-source imagery based on global image matching, Photogrammetric Engineering \& Remote Sensing, 66 (5): 625-629.

Zhou, G., and R. Li, 2000. Accuracy evaluation of ground points from IKONOS high-resolution satellite imagery. Photogrammetric Engineering \& Remote Sensing, 66 (9): 1103-1112. 